\title{
SINGLE- AND TWO-PHASE PERFORMANCE CHARACTERISTICS OF THE MOD-1 SEMISCALE PUMP UNDER STEADY STATE AND TRANSIENT FLUID CONDITIONS
}

\section{D.J. Olson}
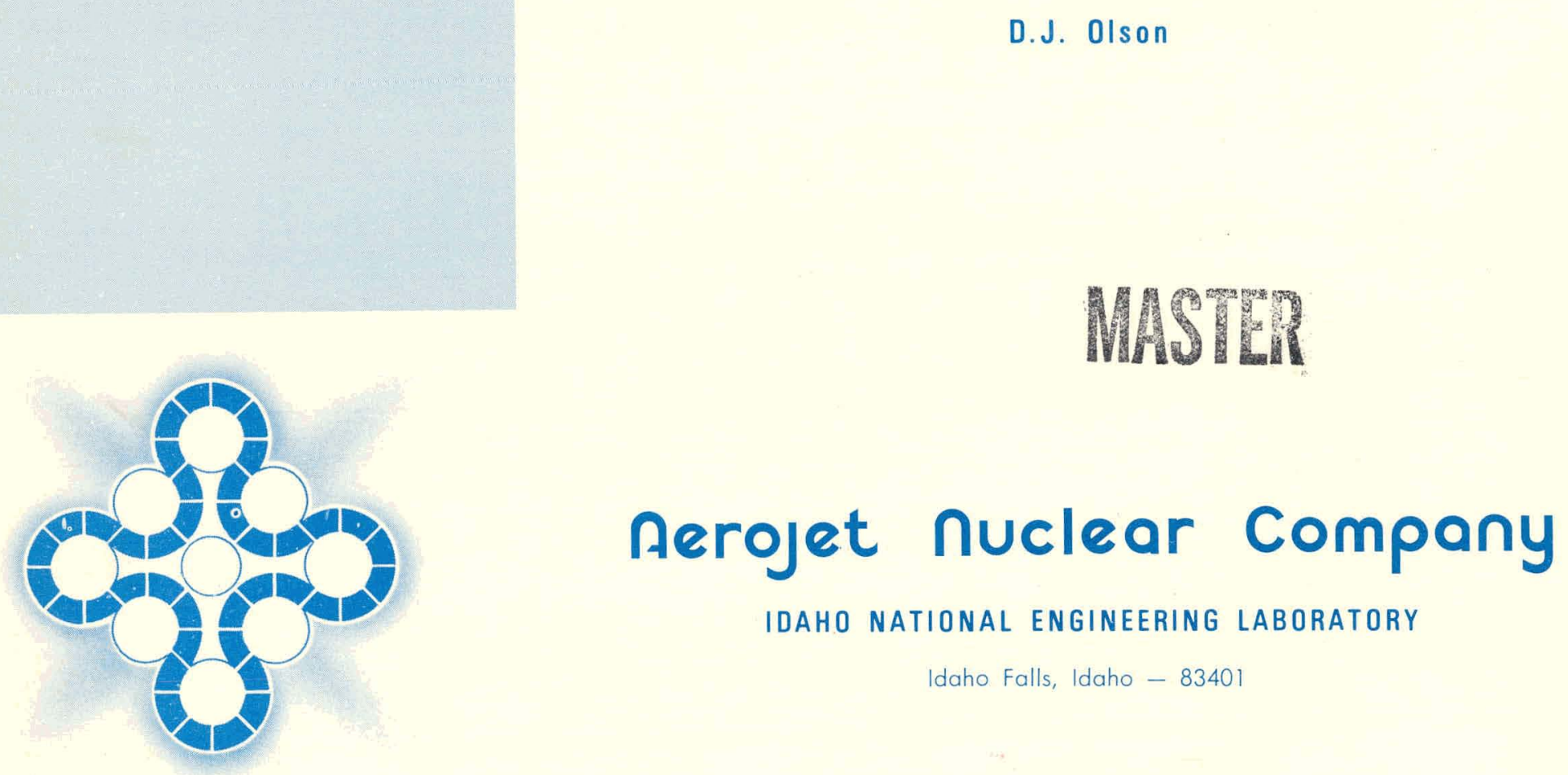

\section{Aerojet nuclear Company}

IDAHO NATIONAL ENGINEERING LABORATORY daho Falls, Idaho -83401 


\section{DISCLAIMER}

This report was prepared as an account of work sponsored by an agency of the United States Government. Neither the United States Government nor any agency Thereof, nor any of their employees, makes any warranty, express or implied, or assumes any legal liability or responsibility for the accuracy, completeness, or usefulness of any information, apparatus, product, or process disclosed, or represents that its use would not infringe privately owned rights. Reference herein to any specific commercial product, process, or service by trade name, trademark, manufacturer, or otherwise does not necessarily constitute or imply its endorsement, recommendation, or favoring by the United States Government or any agency thereof. The views and opinions of authors expressed herein do not necessarily state or reflect those of the United States Government or any agency thereof. 


\section{DISCLAIMER}

Portions of this document may be illegible in electronic image products. Images are produced from the best available original document. 


\section{DISCLAIMER}

This report was prepared as an account of work sponsored by an agency of the United States Government. Neither the United States Government nor any agency Thereof, nor any of their employees, makes any warranty, express or implied, or assumes any legal liability or responsibility for the accuracy, completeness, or usefulness of any information, apparatus, product, or process disclosed, or represents that its use would not infringe privately owned rights. Reference herein to any specific commercial product, process, or service by trade name, trademark, manufacturer, or otherwise does not necessarily constitute or imply its endorsement, recommendation, or favoring by the United States Government or any agency thereof. The views and opinions of authors expressed herein do not necessarily state or reflect those of the United States Government or any agency thereof. 


\section{DISCLAIMER}

Portions of this document may be illegible in electronic image products. Images are produced from the best available original document. 
Printed in the Urited States of America Available trom

National Technical Information Service

U. S. Department of Commerce

5285 Port Royal Road

Springfield, Virginia 22151

Price: Printed Copy $\$ 5.45$; Microfiche $\$ 11,5$

This report was prepared as an account of work sponsored by the United States Government. Neither the United States nor the United States Atomic Energy Commission, nor any of their employees, nor any of their contractors, subcontractors, or their employees, makes any warranty, express or implied, or assumes any legal liability or responsibility for the accuracy, completeness or usefulness of any information, apparatus, product or process disclosed, or represents that its use would not infringe privately owned rights. 
D. J. Olson

\section{AEROJET NUCLEAR COMPANY}

Date Published - October 1974

\section{PREPARED FOR THE U. S. ATOMIC ENERGY COMMISSION}

IDAHO OPERATIONS OFFICE

UNDER CONTRACT NO. AT(10-1)-1375

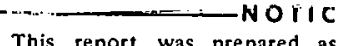




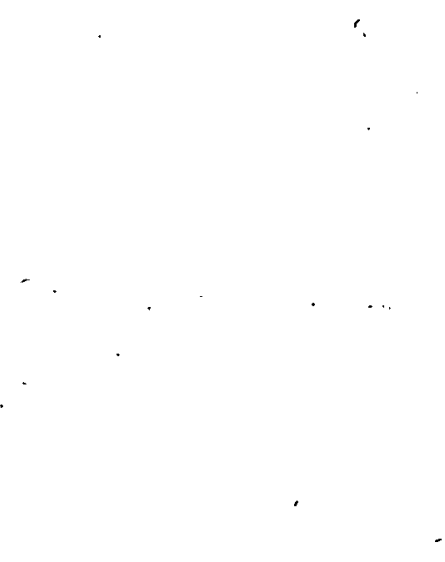

\section{ACKNOWLEDGMENTS}

The author expresses thanks to J. I. Mills and N. A. Edlebeck for their analysis of the effects of fluid inertia on transient two-phase pump performance, to R. W. Shumway for his guidance during the development of the analysis, to K. A. Dietz for editing this report, and to L. K. Keller for typing and correcting the various drafts of this report. 


\begin{abstract}
The performance characteristics of the Mod-1 semiscale pump were evaluated on the basis of data obtained from steady state single- and two-phase flow pump tests performed on the subject pump. In addition, data relative to the response of the Mod-1 pump to transient two-phase fluid conditions were obtained in conjunction with the isothermal blowdown tests conducted as a part of the Semiscale Blowdown and Emergency Core Cooling (ECC) Project. Complete single-phase performance characteristics of the subject pump are presented. Analysis of two-phase results is confined to the normal pump operating region although limited steady state two-phase data are available for abnormal operating regions. Although the analysis resulted in a significant improvement in the understanding of single- and two-phase operating characteristics of the Mod-1 pump, it did not lead to a complete understanding.
\end{abstract}




\section{SUMMARY}

An analysis effort was conducted to establish the performance characteristics of the pump to be used in the intact loop of the Mod-1 semiscale system. The analysis was based on data obtained from steady state single- and two-phase flow pump tests, and on data obtained from transient two-phase flow decompression tests performed as part of 1-1/2-loop scmiscale system isothermal program. The Mod-1 semiscale pump used for these tests is a heavy duty, horizontally mounted, centrifugal pump rated at conditions of $180 \mathrm{gpm}$ against $192 \mathrm{ft}$ of head at $3560 \mathrm{rpm}$ (the specific speed is 926). The steady state tests were conducted to provide pump performance data for each operational mode expected to be encountered in planned loss-of-coolant accident (LOCA) simulations with the Mod-1 semiscale system. The isothermal decompression tests were integral effects tests conducted to investigate the effects of system configuration on ECC performance. Transient two-phase flow data relative to the Mod-1 pump were obtained in conjunction with these tests.

The steady state single-phase flow tests were performed in a simple closed loop recirculation circuit that permitted control of pressure, temperature, and flow and operated with subcooled water at about $90^{\circ} \mathrm{F}$ in the pressure range of 20 to 250 psia. The loop included the necessary piping arrangements to provide for forward and reverse flow through the test pump by means of two recirculation pumps which were available to drive the fluid through the test loop. The two recirculation pumps, each with a capacity of $145,000 \mathrm{lb} / \mathrm{hr}$ at 150 psi pressure rise, could be operated either in parallel or in series thus allowing tests to be performed at conditions in excess of $300 \%$ of the rated capacity of the test pump. The test pump was operated at various impeller speeds in the forward and reverse directions of rotation, with subcooled water flowing from the recirculation system in either the normal or reverse flow direction.

The steady state two-phase flow tests were conducted in a loop that included a steam-water mixer; a steam-water separator, and a recirculation systein. Separate systems were provided for metering and controlling the single-phase steam and water flows to the mixer section. Steam and water were directed to the test loop from a high pressure, oil-fired boiler of sufficient capacity to supply the test loop with up to $30,000 \mathrm{lb} / \mathrm{hr}$ superheated steam or saturated water, or a combined total $30,000 \mathrm{lb} / \mathrm{hr}$ of steam and water at $1000 \mathrm{psia}$. In the two-phase test series the loop was operated at various saturation pressures with a range of steam-water mixtures flowing through the pump in either the forward or reverse direction, and with the pump impeller operated at various speeds in both the forward or reverse direction.

Transient two-phase flow data relative to pump performance were obtained from 1-1/2-loop semiscale isothermal blowdown experiments. The intact loop in the 1-1/2-loop configuration simulates three operating loops of a pressurized water reactor (PWR), and the blowdown loop with simulated pump and steam generator represents the broken loop of a PWR. In each blowdown test evaluated, the semiscale system was heated to about $575^{\circ} \mathrm{F}$, 
pressurized to about 2250 psig, and subjected to a decompression resulting from a rupture in the broken loop. The ratio of break area to system volume in semiscale was equivalent to the ratio of break area to system volume in a four-loop PWR for a double-ended offset shear of a cold leg pipe.

The results of the steady state single-phase tests show that pump head and torque performance data follow the pump affinity relationships quite well. A constant correction of $33 \mathrm{in.}-1 \mathrm{~b}$ to account for bearing and seal friction was required to correlate the torque measurements at different impeller speeds. The homologous head relationships obtained from the single-phase tests were used to obtain the forward and reverse stopped rotor flow resistance. The stopped rotor forward flow resistance, $R^{\prime}$, was $2.91 b_{f}-s e c^{2} / \mathrm{lb}_{\mathrm{m}}-\mathrm{ft}^{3}-\mathrm{in}^{2}$ and the stopped rotor reverse flow resistance was $6.0 \mathrm{lb}_{\mathrm{f}}-\mathrm{sec}^{2} / \mathrm{lb}_{\mathrm{m}}-\mathrm{ft}^{3}-\mathrm{in} .^{2}$.

The results of the steady state two-phase flow tests have been compared with the results from both the steady state single-phase tests and the transient two-phase blowdown tests. The homologous ratios derived from the steady state single-pliase data were found appropriate for predicting the Mod-1 pump behavior for inlet void fractions up to $20 \%$. Comparison of the steady state and transient two-phase flow results for the Mod-1 pump indicates similar behavior for the normal pump operating region. Pump head degradation starts to occur in the range of 16 to $18 \%$ void fraction. Loss of performance occurs rapidly in the range from 18 to $24 \%$ inlet void fraction. Recovery in performance occurs at void fractions greater than about $40 \%$; however, performance is relatively insensitive to inlet void fraction in the range of 30 to $60 \%$.

The analysis of the two-phase flow test results, both steady state and transient, was complicated and the conclusions derived are limited in application because of the inadequacy of measurement instrumentation. Analysis of the data indicates that state-ofthe-art two-phase flow measurements were not adequate for defining fluid conditions at the pump test section if the flow regime encountered was other than homogeneous. In a separated flow condition, the pump reacts to the combined total of the volumetric fluxes of the separated phases. To analyze this condition, both the concentration and velocity profiles of the two-phase mixture must be known. Attempts to compensate for the inadequacies of the instrumentation resulted in the definition of an "effective density" at the pump inlet. This quantity is expressed in terms of the relative velocities of the phases and the average volumetric concentration of an assumed flow regime. This method of analysis significantly improved the correlation of the data points and resulted in pump behavior characteristic curves, in terms of degradation and recovery performance, which are consistent with expected performance. However, the results must be considered qualitative and not an exact definition of the Mod-1 pump characteristics.

Analysis of the available data on the semiscale pump has provided a significant improvement in the understanding of the single- and two-phase pump characteristics. Use of this information will significantly improve pump models used for predictions of semiscale blowdown experiments and the results will be invaluable in the analysis of coupled effects occurring in the semiscale experiments. Although the performance of the semiscale pump is 
not expected to duplicate the performance of PWR pumps because of the differences in size and specific speeds (semiscale Mod-1 pump specific speed of about 1000 compared to PWR pump specific speed of about 5000), the data obtained from the steady slate and transient tests are expected to be useful in evaluating theoretical predictions of centrifugal pump behavior in general and for establishing criteria for future pump tests with larger scale hardware, particularly in the area of instrumentation. In particular, the data obtained indicate that additional pump tests. will be of little value unless either the experimental test loop is designed to ensure the flow to the test pump is homogeneous or two-phase flow measurement methods are developed to quantify density distributions and slip ratios at the pump suction in instances in which a separated two-phase flow condition exists. 


\section{CONTENTS}

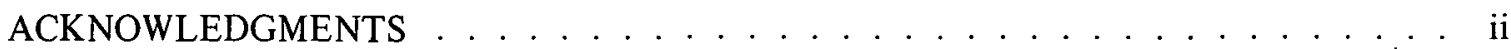

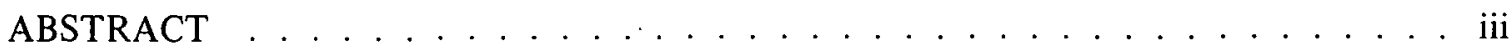

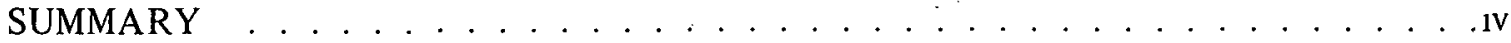

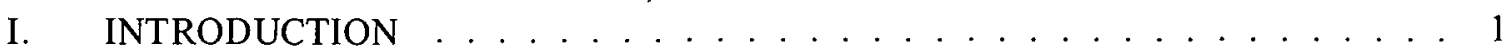

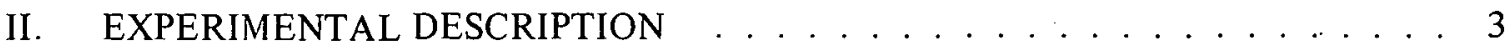

1. STEADY STATE SINGLE-PHASE FLOW TESTS .......... 3

2. STEADY STATE TWO-PHASE FLOW TESTS ............ 5

3. TRANSIENT TWO-PHASE FLOW TESTS .......... 5

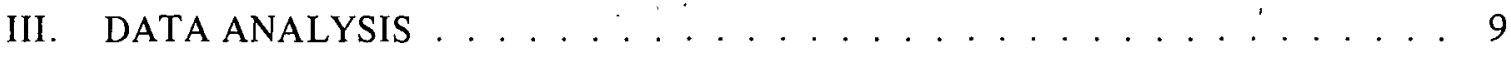

1. SINGLE-PHASE PUMP PERFORMANCE CHARACTERISTICS . . . . . 9

1.1 Head Characteristics . . . . . . . . . . . . . 9

1.2 Torque Characteristics . . . . . . . . . . . . . . 12

1.3 Loss Characteristics . . . . . . . . . . . . . . . . 17

2. STEADY STATE TWO-PHASE PERFORMANCE CHARACTERISTICS . . 22

2.1 Two-Phase Homologous Ratios . . . . . . . . . . . . . . . 22

2.2 Normalized Pump Characteristics -- Head Performance . . . . . . . 24

3. TRANSIENT TWO-PHASE PERFORMANCE ............... 35

3.1 Head Performance . . . . . . . . . . . . . . . . 35

3.2 Torque Characteristics . . . . . . . . . . . . . . . . . . . . . . 3.7

IV. SUMMARY OF OBSERVATIONS AND CONCLUSIONS . . . . . . . . . . . 40

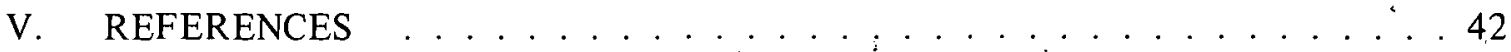

APPENDIX A -- PRESENTATION OF TRANSIENT DATA FROM SEMISCALE

ISOTHERMAL BLOWDOWN TESTS $" \ldots \ldots . \ldots . \ldots . \ldots . \ldots$

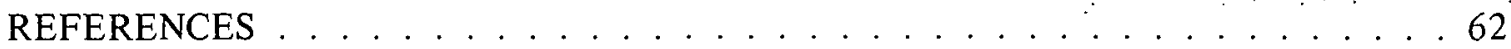

APPENDIX B -- THE EFFECTS OF FLOW REGIME ON MEASURED DENSITY AND THE METHOOD UT UB I'AINING AN EFFECTIVE DENSITY AT THE

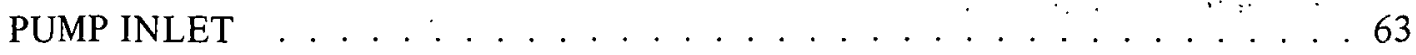


1. FLOW REGIME AND MEASURED DENSITY . . . . . . . . . . . . . 65

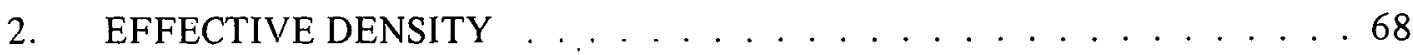

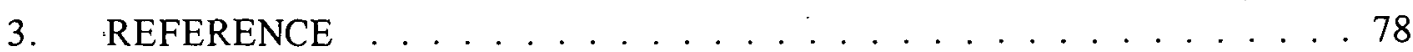

THE EFFECTS OF IMPELLER FLUID INERTIA $\ldots \ldots . . \ldots$

\section{FIGURES}

1. Steady state single-phase test loop .................. . . 4

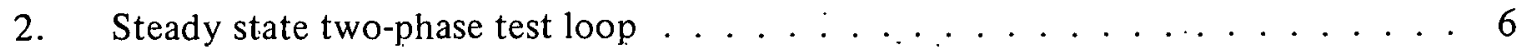

3. 1-1/2-loop semiscale isothermal test system . . . . . . . . . . 7

4. Steady state single-phase normalized pump characteristic . . . . . . . . . 10

.5. Single-phase four-quadrant head curves for the Mod-1 semiscale pump . . . . . 11

6. Single-phase homologous head curve for the Mod-1 semiscale pump . . . . . . . 12

7. Single-phase homologous torque curve for the Mod-1 semiscale pump (uncorrected) . . . . . . . . . . . . . 13

8. Single-phase homologous torque curves for pump speeds of 1600 and $2700 \mathrm{rpm} \ldots \ldots \ldots \ldots \ldots \ldots$

9. Single-phase normal torque characteristics . . . . . . . . . . . 15

10. Single-phase homologous torque curve for the Mod-1 semiscale pump (corrected for frictional torque) . . . . . . . . . . . . . 17

11. Single-phase four-quadrant torque curves for the Mod-1 semiscale pump . . . . . . 18

12. Pump operation and losses with reference to homologous head curve ...... 21

13. Forward flow resistance for the Mod-1 pump . . . . . . . . . . . 22

14. Reverse flow resistance for the Mod-1 pump . . . . . . . . . . . . . 23

!15. Two-phase homologous ratios . . . . . . . . . . . . . . . 24

16. Two-phase pump characteristics $-0 \leqslant \alpha \leqslant 0.25 \ldots \ldots 26$

17: Two-phase pump chàracteristics $-0.25 \leqslant \alpha \leqslant 0.50 \ldots \ldots \ldots \ldots$ 
18. Two-phase pump characteristics $-0.50 \leqslant \alpha \leqslant 0.75 \ldots \ldots . \ldots 28$

19. Two-phase pump characteristics $-0.75 \leqslant \alpha \leqslant 1.0 \ldots \ldots$. . . . . . . . . 29

20. Two-phase pump characteristic corrected for flow regime effects $-0 \leqslant \alpha \leqslant 0.50 \ldots \ldots \ldots \ldots$. . . . . . . . . . . . . . . . .

21. Two-phase pump characteristics corrected for flow regime effects $-0.50 \leqslant \alpha \leqslant 1.0 \ldots \ldots \ldots . \ldots \ldots 2$

22. Steady state two-phase degradation performance as a function of inlet void fraction . . . . . . . . . . . . . . . . . . . . . . . . .

23. Steady state two-phase recovery performance as a function of inlet void fraction . . . . . . . . . . . . . . . . 34

24. Transient two-phase pump performance data - Test $1009 \ldots \ldots 6$

25. Comparison of steady state and transient two-phase pump performance $\ldots . .38$

26. Transient two-phase torque characteristics - Test $1009 \ldots \ldots$. . . . . . . 39

A-1. Pump differential pressure -- Test $1008 \ldots \ldots . \ldots . \ldots . \ldots$

A-2. Pump speed -- Test $1008 \ldots \ldots$. . . . . . . . . . . . . . . . . . . . . . . . . .

A-3. Pump torque -- Test $1008 \ldots \ldots$. . . . . . . . . . . . . . . . . . . . . . . .

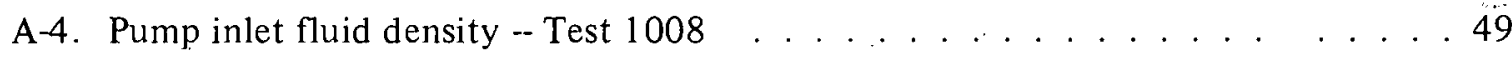

A-5. Pump inlet void fraction -- Test $1008 \ldots \ldots$. . . . . . . . . . 50

A-6. Pump inlet volumetric flow rate -- Test $1008 \ldots \ldots$. . . . . . . . . 50

A-7. Pump outlet fluid density -- Test $1008 \ldots \ldots \ldots \ldots \ldots$

A-8. Pump outlet void fraction - Test $1008 \ldots \ldots \ldots \ldots$

A-9. Pump outlet volumetric flow rate -- Test $1008 \ldots \ldots \ldots$. . . . . . . . . 52

A-10. Pump differential pressure -- Test $1009 \ldots \ldots \ldots$. . . . . . . . . 52

A-11. Pump speed - Test $1009 \ldots \ldots$. . . . . . . . . . . . 53

A-12. Pump torque -- Test $1009 \ldots \ldots \ldots \ldots$

A-13. Pump inlet void fraction - Test $1009 \ldots \ldots \ldots \ldots$

A-14. Pump inlet void fraction -- Test $1009 \ldots \ldots$. . . . . . . . . . 54 
A-15: Pump inlet volumetric flow rate - Test $1009 \ldots \ldots 5$

A-16. Pump outlet fluid density -- Test $1009 \ldots \ldots$. . . . . . . . . . . . . . .

A-17: Pump outlet void fraction - Test $1009 \ldots \ldots 6$

$\mathrm{A} \div 18$. Pump outlet volumetric flow rate - Test $1009 \ldots \ldots 6$

$\mathrm{A}=19$. Pump differential pressure -- Test $1010 \ldots \ldots$. . . . . . . . . . . . .

A-20: Pump speed-- Test $1010 \ldots \ldots \ldots \ldots$

A-21. Pump torque - Test $1010 \ldots \ldots \ldots$

A-2.2. Pump inlèt fluid density - Test $1010 \ldots \ldots$. . . . . . . . . 58

A-23. Pump inlet void fraction -- Test $1010 \ldots \ldots \ldots$. . . . . . . . . . . . .

A-24. Pump inlet volumetric flow rate -- Test $1010 \ldots \ldots$. . . . . . . . . 59

A-25. Pump outlet fluid density -- Test $1010 \ldots \ldots$. . . . . . . . . . 60

A-26: Pump outlet void fraction - Test $1010 \ldots \ldots$. . . . . . . . . . . . . . .

A-27. Pump outlet volumetric flow rate - Test $1010 \ldots \ldots 1$

B-1 Representation of annular flow in pipe . . . . . . . . . . . 65

B-2. Path traversed by gamma beam through pipe containing annular flow

B-3. Fluid density and void fraction versus film thickness for pure annular flow . . . . . . . . . . . . . . . . 68

B-4. Fluid density and void fraction versus liquid height for pure stratified flow . . . . . . . . . . . . . . . . . . . . . . 69

\section{TABLES:}

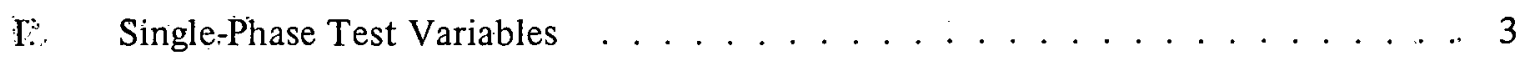

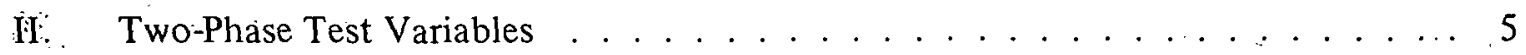

B:I Flow Regime Type from Effective Density Method . . . . . . . . . . 73 
C-I. Pump Impeller, Inlet Line, Discharge Line Geometry, and Transient Inertia Head for the Mod-1 Semiscale Pump

C-II. Comparison of Impeller and Pump Piping Fluid Inertia Heads in Semiscale System . . . . . . . . . . . . . . . . 85 


\section{SINGLE- AND TWO-PHASE PERFORMANCE CHARACTERISTICS}

OF THE MOD-1 SEMISCALE PUMP UNDER

STEADY STATE AND TRANSIENT FLUID CONDITIONS

\section{INTRODUCTION}

An extensive water-cooled nuclear reactor safety research program is currently sponsored by the United States Atomic Energy Commission to develop and assess the capability of analytical models for predicting system behavior during various hypothesized accident situations. The Semiscale Blowdown and Emergency Core Cooling (ECC) Project is one of the system effects type experiments being sponsored to simulate loss-of-coolant accident (LOCA) behavior of pressurized water reactors (PWR). The primary objective of the semiscale program is to provide coupled thermal-hydraulic data for use in the development and evaluation of analytical models. The small size of the components and the use of electrical heaters to simulate the performance of nuclear fuel precludes valid use of the results for demonstrational purposes. However, the semiscale experimental results, through comparison with data from other experiments in larger geometries, are expected to provide a basis for evaluating the effects of physical scale. The complexities of the interrelated phenomena occurring during a simulated LOCA require that separate effects tests be performed on some components in order to more fully understand the individual contribution of these components. The steady state single- and two-phase tests, described, were specifically performed to develop an understanding of the influence of the Mod-1 semiscale pump.

The pump in the intact loop of the Mod-1 semiscale system has major influence on system behavior during decompression. The ability to predict pump performance over the complete void fraction range, 0 to $100 \%$, is required to understand the interrelated hydraulic phenomena controlling loop and core flow rates during LOCA simulations. Of particular importance is the head degradation and recovery under two-phase flow conditions as a function of void fraction and pump speed.

Data in the literature relative to the operation of centrifugal pumps subjected to steam-water mixtures are very. limited. Most data are for low pressure, low quality conditions, or for two-phase, two-component mixtures (usually air-water mixtures). General Electric Company has conducted some steady state, high pressure, steam-water tests on a single-stage vertical centrifugal pump (specific spced 1600 ) from 0 to $30 \%$ void fraction [1]. They have also performed transient and steady state tests on a small high head $(680 \mathrm{ft})$ pump in the normal operating region to void fractions of about $40 \%$ [2]. Because of the high head rise of the General Electric Company pumps, the data correlations obtained cannot be used to characterize the performance of the relatively low head semiscale pump during 
LOCA simulations. The semiscale pump is a horizontally mounted centrifugal pump with rated conditions of $180 \mathrm{gpm}$ against $192 \mathrm{ft}$ of hcad at $3560 \mathrm{rpm}$. The pump has a specific speed of 926.

Steady state tests were run on the semiscale Mod+1 pump to obtain the single- and two-phase performance characteristics throughout all regions of pump operation and for the full void fraction range from 0 to $100 \%[3]$. Performance data on the same pump are also availäble for a-transient decompression as a result of the pump being.used as the intact loop pump for the 1-1/2-loop semiscale system during the isothermal blowdown tests ${ }^{[4-9]}$.

This report presents an evaluation of the steady state and transient data obtained for the Mod-1 semiscale pump. A brief description of the experimental apparatus used for each type of test is presented and the range of test variables is identificd. The approach followed in the data analysis was to evaluate the single-phase steady state data relative to expected performance derived from pump affinity laws. Four-quadrant characteristic curves and homologous curves were subsequently developed from the single-phase data to establish performance characteristics for all hydraulic conditions of interest in LOCA simulations. The homologous curves were then used to derive the forward and reverse flow locked rotor resistance of the pump. The steady state two-phase data were compared-with the single-phase performance characteristics curves to establish the influence of void fraction at the pump inlet.

The inadequacy of the two-phase fluid state measurements for other than a homogeneous flow regime complicated the analysis. To correlate much of the two-phase data a method was derived to reevaluate the density measurements assuming separated flow and specific pump inlet conditions so as to compensate for individual phase velocities. The transient two-phase data from the isothermal blowdown tests were compared with the two-phase performance curves developed from the data obtained from the steddy state two-phase flow pump tests.

The analysis has resulted in a significant improvement in the understanding of the single- and two-phase performance characteristics of the Mod-1 pump This understanding will enhance the analysis of coupled effects occurring in the Mod-1 semiscale experiments and will improve pump models used for predictions of blowdown experiments. The difficulties encountered in the analysis of the results of the steady state and transient two-phase flow tests because of the influence of flow regime emphasize the need for better. measurement techniques and provide guidance for the conduct of future pump tests. 


\section{II . EXPERIMENTAL DESCRIPTION}

The data evaluated in this report were obtained from three distinct experimental efforts: (a) steady state single-phase pump performance tests, (b) steady state two-phase pump performance tests, and (c) isothermal blowdown tests in the 1-1/2-loop semiscale system. In each of these experimental efforts the subject pump was the pump to be used in the intact loop of the 1-1/2-loop Mod-1 semiscale system. The semiscale pump is a heavy duty horizontally mounted centrifugal pump which was modified for service in the Mod-1 system as outlined in Reference 10. These modifications consisted of a 7-3/4-inch diameter impeller and a venturi in the pump discharge port. The modifications resulted in rated conditions of $180 \mathrm{gpm}$ against $192 \mathrm{ft}$ of head at $3560 \mathrm{rpm}$. The rated torque on the pump shaft at these conditions is $417.6 \mathrm{in}$.-lb and the specific speed is 926 .

A summary of each of the three experimental efforts follows. A more detailed description of the test procedures and test conditions in all three efforts can be found in References. 3 and 11. Reference 3 also provides summary tables of the data taken during the steady state tests and a description of data reduction methods and instrument errors. Data from the isothermal blowdown tests have also been reported ${ }^{[4-9]}$.

\section{STEADY STATE SINGLE-PHASE FLOW TESTS}

The single-phase tests provided pump performance data for all possible pump operational modes. The test variables included pressure, fluid temperature, flow rate in both the forward and reverse flow directions, and impeller speed in both the forward and reverse directions. The range of each test variable is given in Table I.

The tests were performed with subcooled water in the pressure range of 20 to 250 psia in a simple closed loop recirculation circuit which permitted control of pressure,

$\underline{\text { TABLE I }}$

SINGLE-PHASE TEST VARIABLES

\begin{tabular}{ll} 
Pressure & 0 to $200 \mathrm{psig}$ \\
Fluid temperature & 75 to $125^{\circ} \mathrm{F}$. \\
Flowrate & -200 to $350 \mathrm{gpm}$ \\
Impeller speed & -2700 to $2700 \mathrm{rpm}$ \\
\hline
\end{tabular}


temperature, and flow. The single-phase test loop and test pump section are shown isometrically in Figure 1. The loop included the necessary piping arrangements to allow forward and reverse flow through the test pump. Two recirculation pumps, each with a capacity of $145,000 \mathrm{lb} / \mathrm{hr}$ at 150 psi pressure rise, were used to drive the fluid through the test loop. The two pumps could be operated either in parallel or in series, thus allowing tests to be performed at conditions in excess of $300 \%$ of the rated capacity of the test pump.

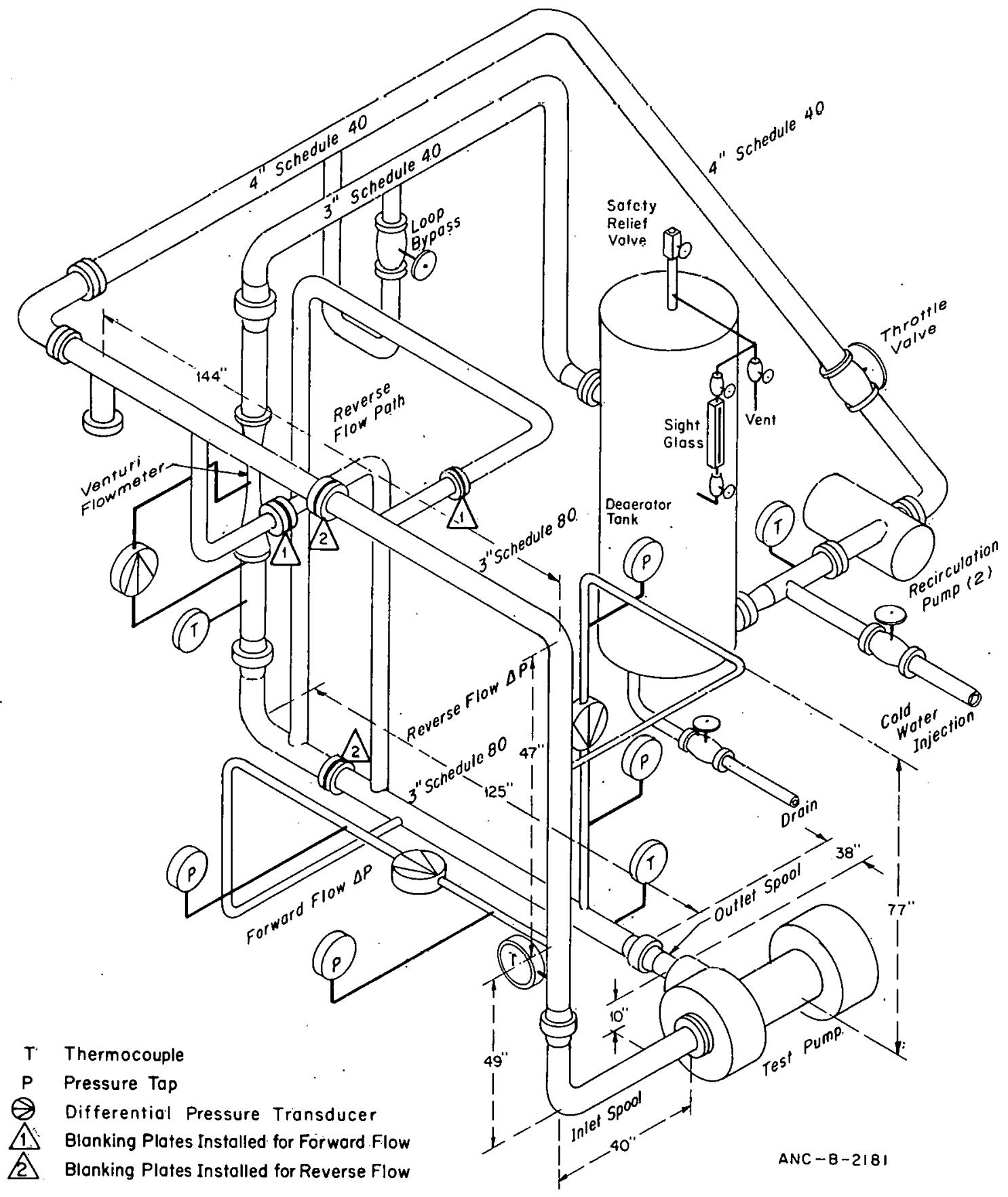

Fig. 1 Steady state single-phase test loop. 


\section{STEADY STATE TWO-PHASE FLOW TESTS}

The steady state two-phase test loop is shown isometrically in Figure 2. The two-phase test loop, particularly that portion containing the test section, was basically the same as that used during the single-phase tests. This loop included a steam-water mixer, a steam-water separator, and a recirculation system. Additionally, the two-phase test loop included provisions for metering and controlling the single-phase steam and water inputs to the test loop separately. Steam and water were directed to the test loop from a high pressure, oil-fired boiler of sufficient capacity to supply the test loop with up to $30,000 \mathrm{lb} / \mathrm{hr}$ superheated steam or saturated water, or a combined total of $30,000 \mathrm{lb} / \mathrm{hr}$ of steam and water at 1000 psia. The loop was operated at various saturation pressures with a range of steam-water mixtures flowing through the pump in either the forward or reverse direction, and with the pump impeller rotating in the forward or reverse direction. The test variables and the ranges investigated are summarized in Table II.

TABLE II

TWO-PHASE TEST VARIABLES

$\begin{array}{ll}\text { Pressure } & 200,500, \text { and } 900 \text { psia } \\ \text { Fluid temperature } & 375 \text { to } 535^{\circ} \mathrm{F} \\ \text { Flowrate } & -200 \text { to } 350 \mathrm{gpm} \\ \text { Impeller speed } & -1600 \text { to } 2700 \mathrm{rpm} \\ \text { Void fraction (homogeneous) } & 0 \text { to } 100 \%\end{array}$

Summary tables of data taken during the two-phase tests and a description of data reduction methods and instrument errors can be obtained from Reference 3 .

\section{TRANSIENT TWO-PHASE FLOW TESTS}

Transient two-phase performance data for the pump were obtained during the 1-1/2-loop semiscale isothermal blowdown tests. The semiscale isothermal system is illustrated in Figure 3. The system is a small-scale representation of a four-loop PWR system in which the intact loop is designed to simulate performance in three unbroken loops of the four-loop system and includes a pressurizer, steam generator, primary coolant pump, and the necessary piping. The components in the fourth loop of the reference PWR are 


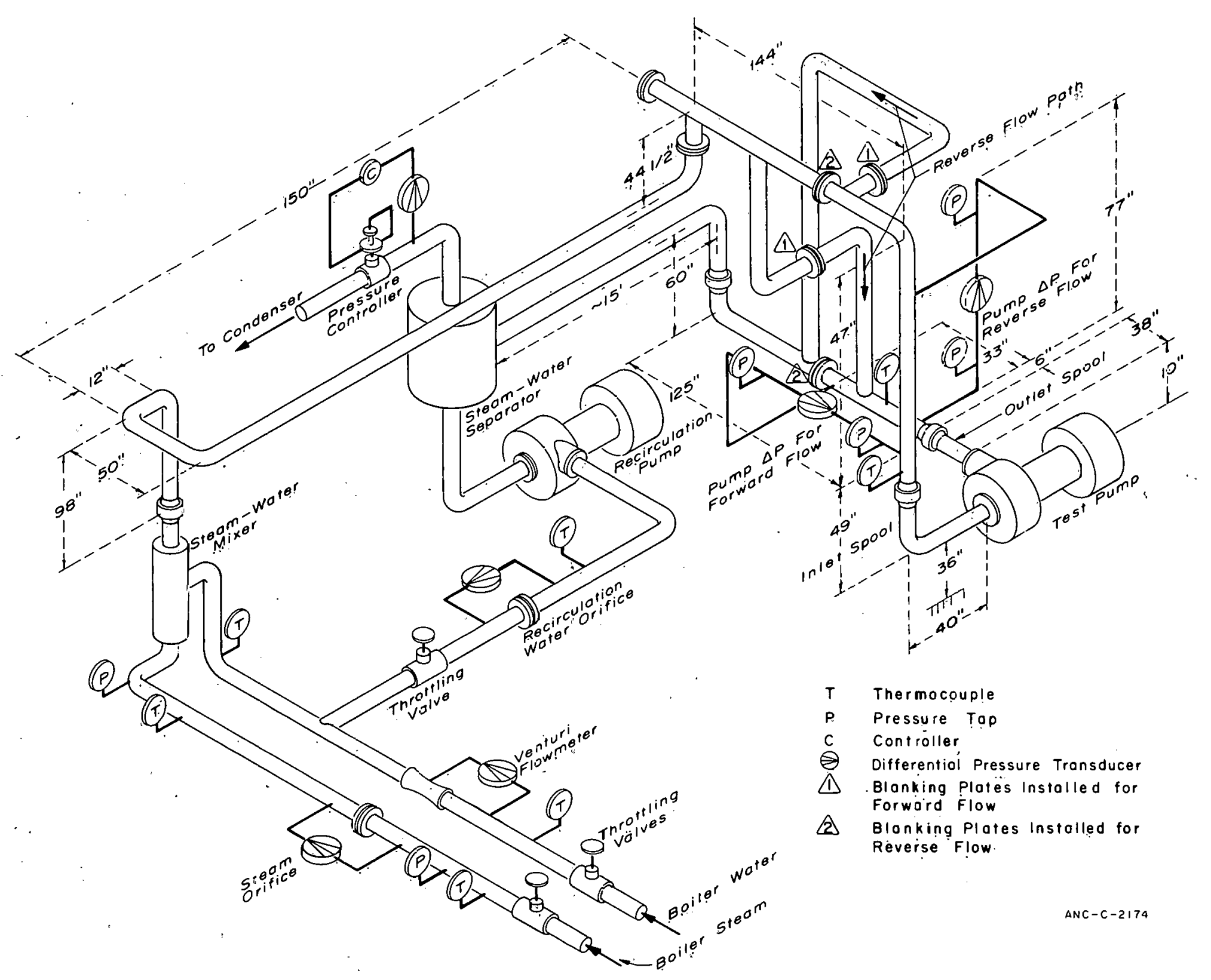

Fig. 2 Steady state two-phase test loop. 


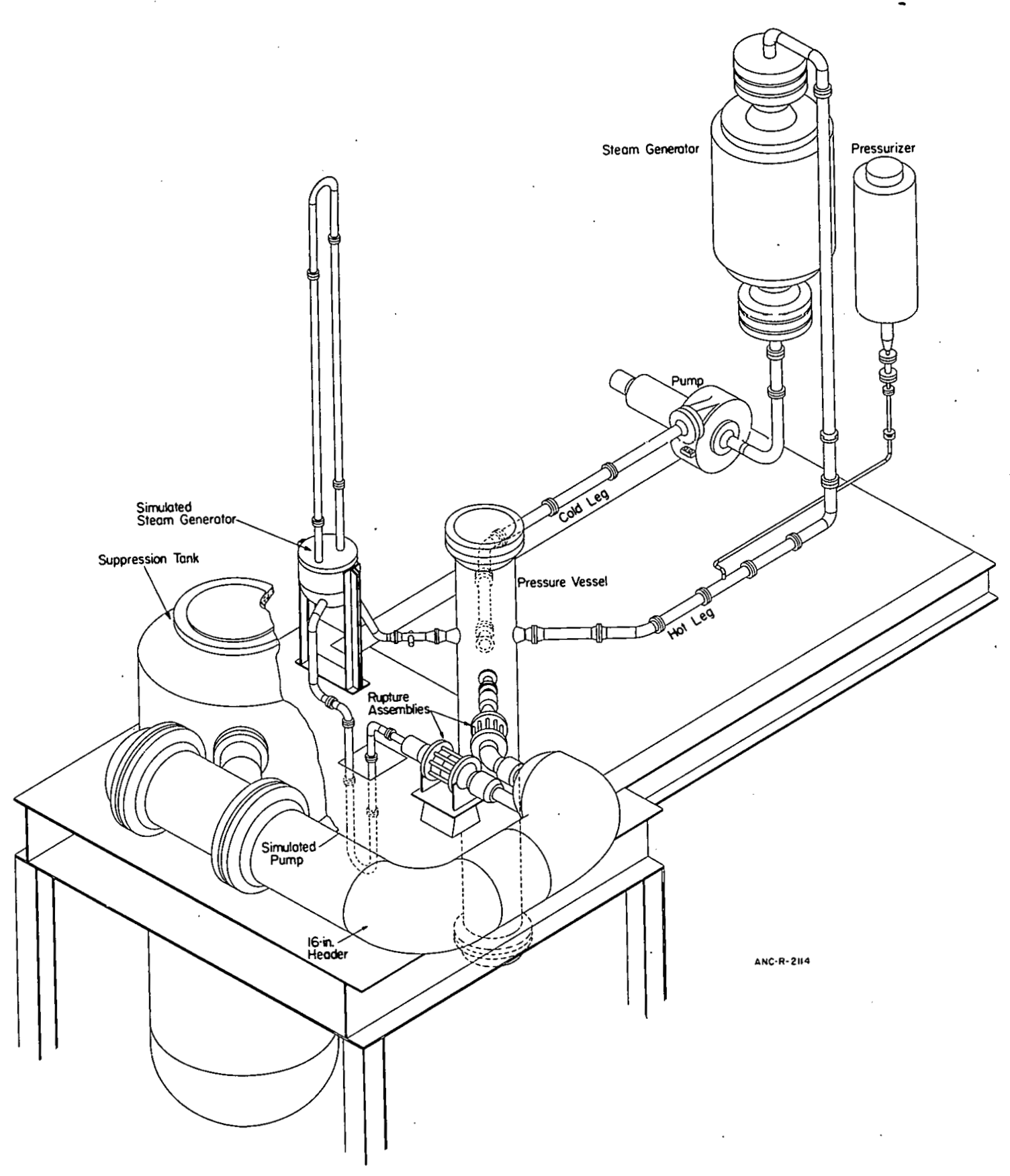

Fig. 3 1-1/2-loop semiscale isothermal test system. 
simulated in the: blowdown loop. The semiscale pressure vessel includes upper and lower plenums, core barrel, and a downcomer. A pressure suppression system, which includes a tank: and a header, is attached to the semiscale blowdown loop to simulate the backpressure of a containment building during the course of a simulated LOCA.

The isothermal tests consisted of blowdown tests initiated from nominal conditions of 2250 psig and $577^{\circ} \mathrm{F}$. In all of these tests the primary coolant pump (1-1/2-loop Mod-1 semiscale pump) remained under power for the duration of the test. The pump was belt-driven through an integral torque meter by a $1: 5 \mathrm{hp}$, variable speed, dc electric motor: A speed controller was used to maintain a relatively constant pump speed throughout the tests: The nominal initial pump condition for all tests was 170 gpm against $127 \mathrm{ft}$ of: head at 3000 rpm: Results from each of the isothermal tests have been reported separately in experiment data reports [4-9] that give a complete description of test conditions and inclide all recorded data obtained from each test. Data pertinent to the evaluations presented in this. report have been included in Appendix A. A summary of detector characteristics and measurement system errors for instruments used in the isothermal program is available $[1.1]$. 


\section{III . DATA ANALYSIS}

Analysis of the data obtained from each of the three testing efforts is presented in three separate discussions. The analysis establishing the single-phase characteristics is presented first, the analysis of steady state and transient two-phase results related to the single-phase results is presented next, and the analysis of transient two-phase results related to the steady state two-phase results is presented last.

\section{SINGLE-PHASE PUMP PERFORMANCE CHARACTERISTICS}

Single-phase performance characteristics of centrifugal pumps in the normal pump operational mode have been thoroughly investigated experimentally and theoretically and are well documented in numerous text books. The experimental and theoretical developments have indicated that the head and cupacity of a centrifugal pump vary with the speed of the pump in such a way that the performance curves retain their characteristic features. The variation of pump head and flow at a constant speed is referred to as the pump characteristic. Pump speed, pump capacity, and pump head are related by mathematical expressions referred to as affinity laws. Basically, the affinity laws state that capacity varies directly as the pump speed, and head varies directly as the square of the pump speed. These relationships have been used in the analysis of the steady state single-phase data to normalize head-capacity curves for various impeller speeds. The normalized semiscale pump characteristic derived from the single-phase data is shown in Figure 4. This curve shows that the measured head flow data follow pump affinity relationships quite well.

Characterizing single-phase pump behavior for all other operating modes of interest for LOCA evaluation requires considerably more information than is provided by the normalized characteristic curve shown in Figure 4. Performance curves which describe all possible operating characteristics of a centrifugal pump are generally referred to as four-quadrant characteristic curves. These curves are lines of constant head and torque plotted as functions of both positive and negative values of volumetric flow rate and impeller speed. A complete description of the experimental and theoretical development of four-quadrant characteristic curves can be found in Reference 12. The type and number of data points generated during the single-phase tests were selected to establish the complete four-quadrant performance characteristics of the 1-1/2-loop Mod-1 semiscale pump. For clarity and ease of presentation, the performance characteristics, in terms of head and torque, are presented separately.

\subsection{Head Characteristics}

The experimentally determined four-quadrant characteristics head. curve for the 1-1/2-loop Mod-1 semiscale pump is presented in Figure 5. Obtaining the lines of constant head, $H$, on this curve requircd interpulation between data points. The expected 


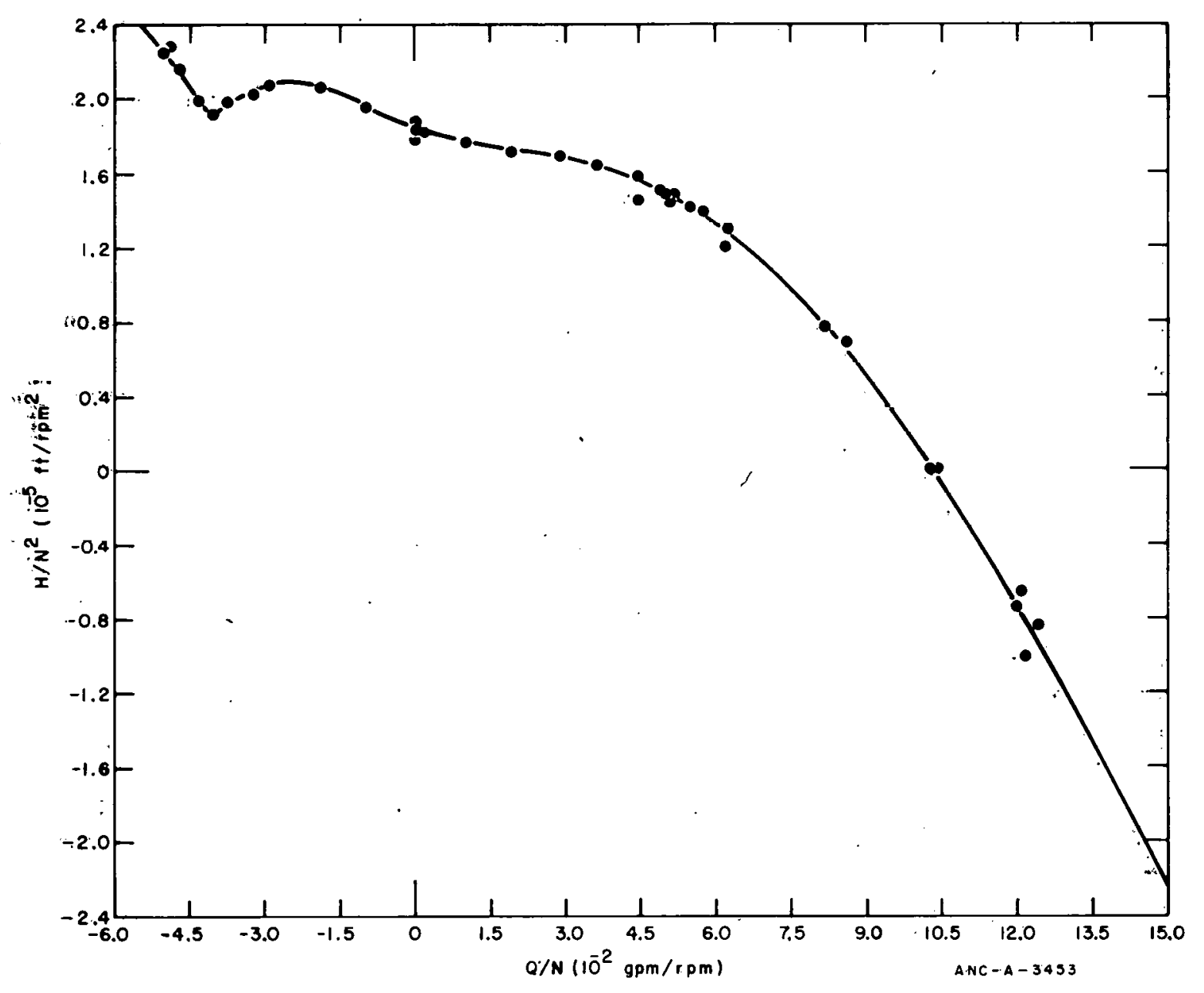

Fig. 4. Steady statc single-phase normalized pump characteristic,

performance of the semiscale pump for any possible operating condition with single-phase fluid as the working medium can be obtained from the results presented in this figure.

For computer analysis, characterizing pump performance in terms of homologous curves $^{[13]}$ (in which a family of curves can be represented by one curve segment) is useful because of the relative ease of tabulating the required input data. The homologous curves are dimensionless extensions of the four-quadrant pump characteristic curves in which the -parameters $\mathrm{H}$ (head) or $\mathrm{T}$ (torque), $\mathrm{Q}$ (capacity), and $\mathrm{N}$ (impellcr speed) are made :dimensionless through division by the appropriate rated (point of maximum efficiency) parameter. The homologous curves are actually two sets of four dimensionless characteristic curve segments that are plotted on the same set of coordinates. In one set, the dimensionless "head ratio $\left(\mathrm{h}=\mathrm{H} / \mathrm{H}_{\mathrm{r}}\right)$ or torque ratio $\left(\beta=\mathrm{T} / \mathrm{T}_{\mathrm{r}}\right)$ as a function of the dimensionless flow $(v=$ $\left.Q \mathrm{Q} / \mathrm{Q}_{\mathrm{r}}\right)$ is plotted versus the dimensionless speed $\left(\alpha=N / N_{r}\right)$, which also is a function of dimensionless flow; that is, $\mathrm{h} / v^{2}$ (or $\beta / v^{2}$ ) is a function of $\alpha / v$. In the second set, the $\therefore$ dimensionless head or torque ratio as a function of dimensionless speed is plotted versus the : dimensioniless flow as a function of dimensionless speed; that is, $h / \alpha^{2} \cdot\left(\operatorname{or} \beta / \alpha^{2}\right)$ is a function 


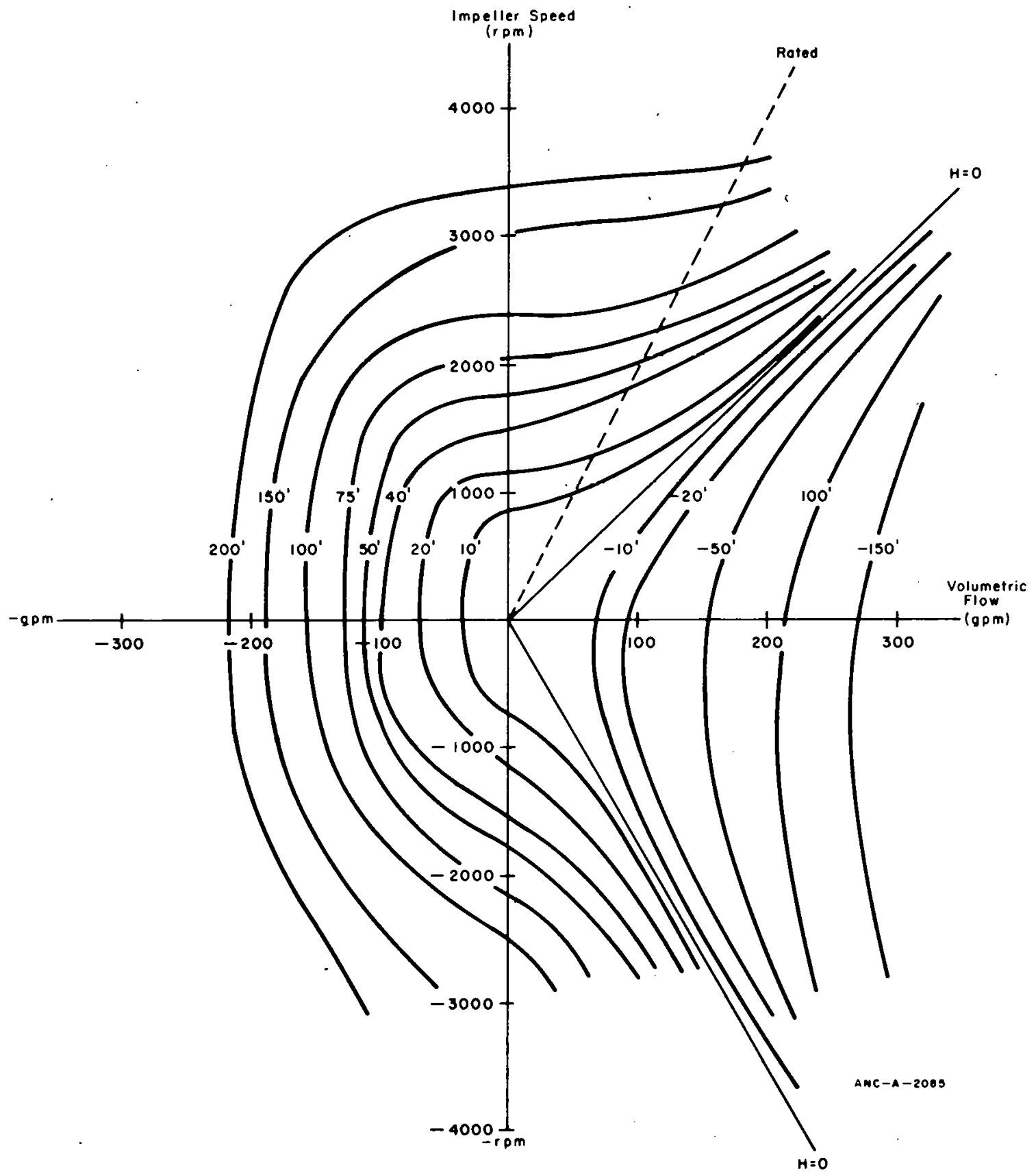

Fig. 5 Single-phase four-quadrant head curves for the Mod-1 semiscale pump.

of $v / \alpha$. In this manner, the complete pump characteristics through all zones of operation can be representcd by an intersecting set of eight curve segments, one set for the head characteristics and one set for the torque characteristics.

The experimentally developed single-phase homologous head çurve is shown in Figure 6. A three-letter acronym is used to designate the curve type. H refers to head ratio, 

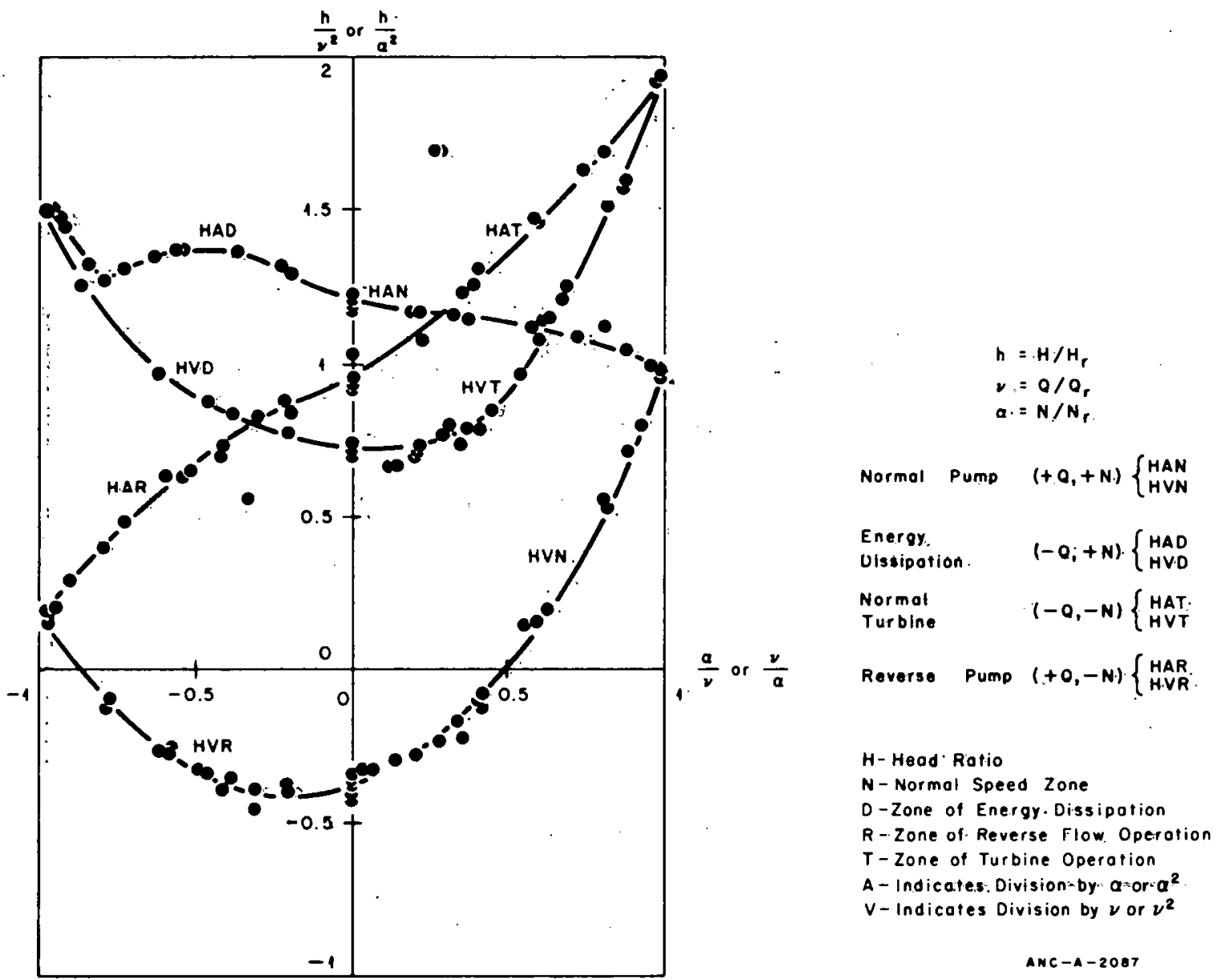

Fig. 6 Single-phase homologous head curve for the Mod-1 semiscale pump.

A indicates division by $\alpha$ or $\alpha^{2}, \mathrm{~N}$ indicates the normal pump zone, $\mathrm{V}$ indicates division by $v$ or $v^{2}, \mathrm{D}$ is the zone of energy dissipation, $\mathrm{R}$ is the zone of reverse flow pump operation, and $\mathrm{T}$ is the zone of turbine operation. All data points obtained from the single-phase tests are shown on Figure 6. The correlation of the data over the wide range of variables investigated is good. The results of the single-phase tests indicate that the operational characteristics of the pump in the normal operating regions (regions HAN and HVN of the homologous curve of Figure 6) are close to the design conditions specified by the methods of Reference 10 . The operation in the abnormal regions [energy dissipation (HAD, HVD), turbine opcration (HAT, HVT); and reverse pump operation (HAR, HVR)] was not specified; however; the results are similar to what might be expected from a low specific speed pump. Streeter and Wylie present, as an example, the homologous curve for a pump with a specific speed of $1800^{[13]}$ :

\subsection{Torque Characteristics}

Since the homologous curves are based on the hydraulic performance of a pump, the measured torque values have to be corrected for frictional torque if that torque is a 
significant part of the total measured torque. Friction losses in a pump result from bearing and seal losses and from disc friction. The value of these losses is influenced by a number of factors, such as pressure, pump speed, method of lubrication, and load. In large pumps these combined losses are generally a small fraction of the total torque generated and, therefore, are negligible; however, whether or not these losses were negligible for the small Mod-1 pump was not known.

Figure 7 presents the result of the single phase torque measurements in terms of the homologous torque characteristics and indicates rather significant data scatter. The torque

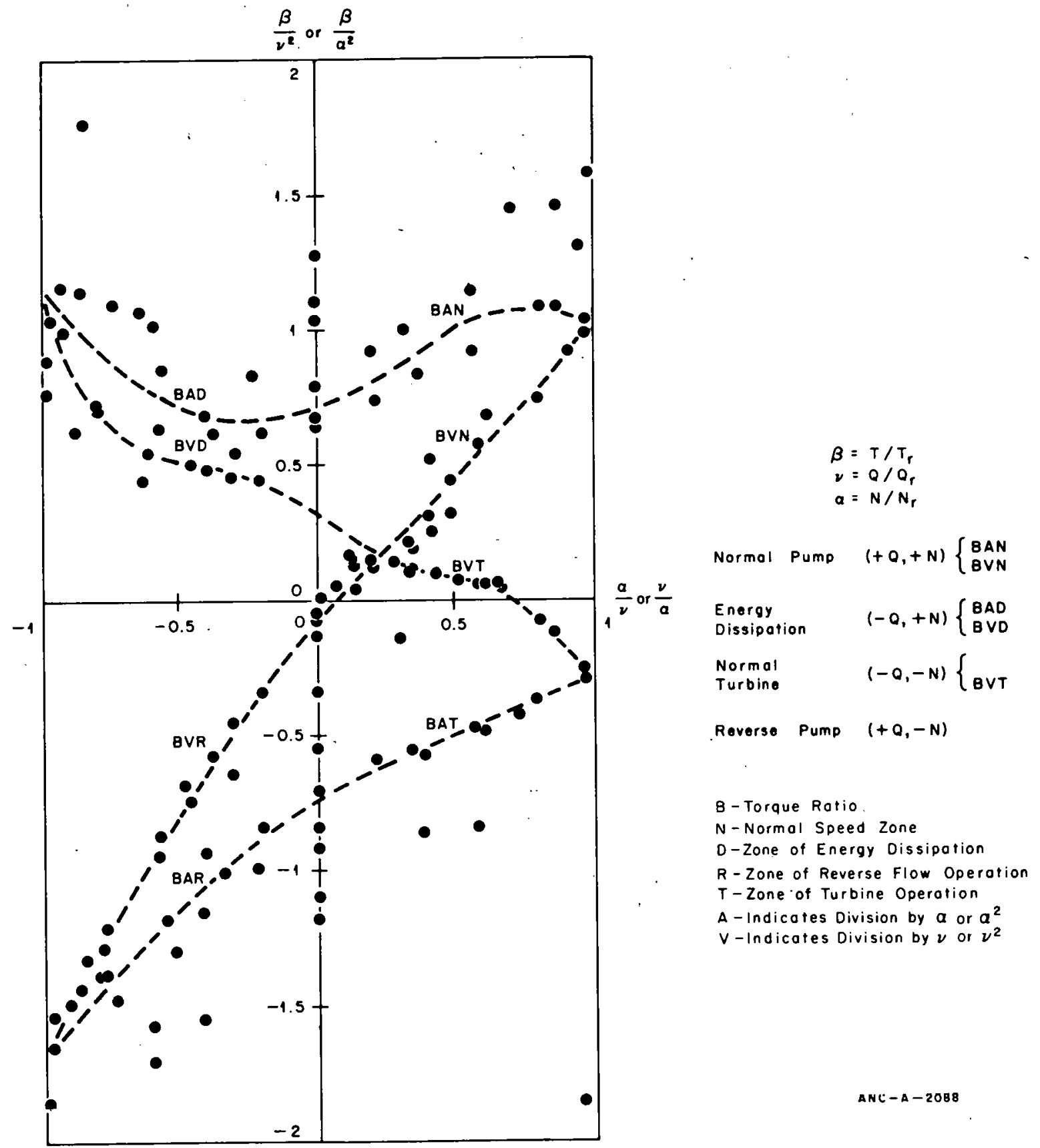

Fig. 7 Single-phase homologous torque curve for the Mod-1 semiscale pump (uncorrected). 
data in Figure 7 for tests conducted at \pm 2700 and $\pm 1600 \mathrm{rpm}$ have been replotted in Figure 8. This figure shows clearly that frictional torque must be accounted for in the measured

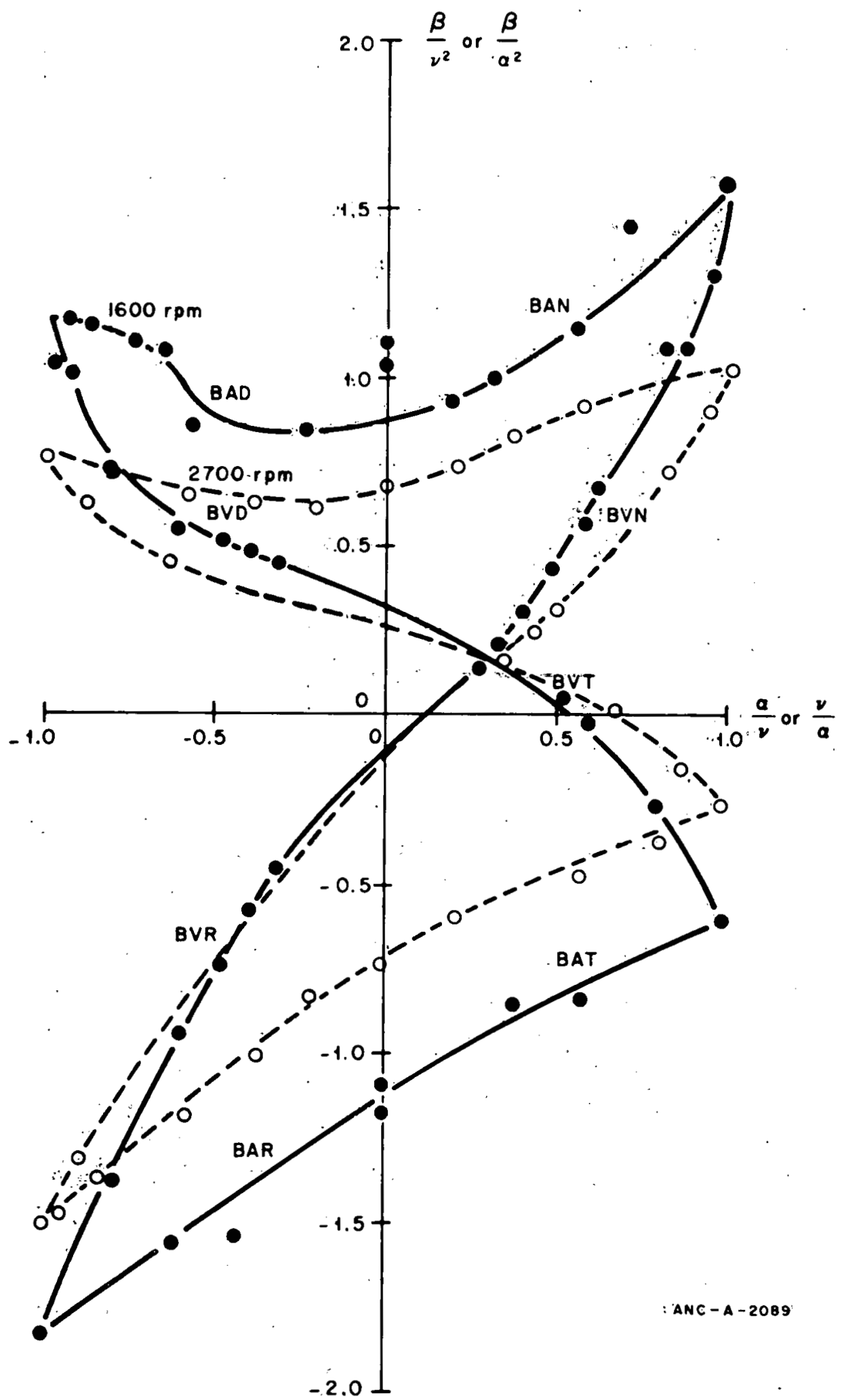

Fig. 8 Single-phase homologous torque curves for pump speeds of 1600 and $2700 \mathrm{rpm}$. 
values to correlate data at the various pump speeds. Since the analysis of the head characteristics indicated that the subject pump follows the affinity relationships quite well, an indication of the value of the frictional torque can be obtained by plotting the torque data on a normalized basis, as shown in Figure 9. In this figure the measured torque divided

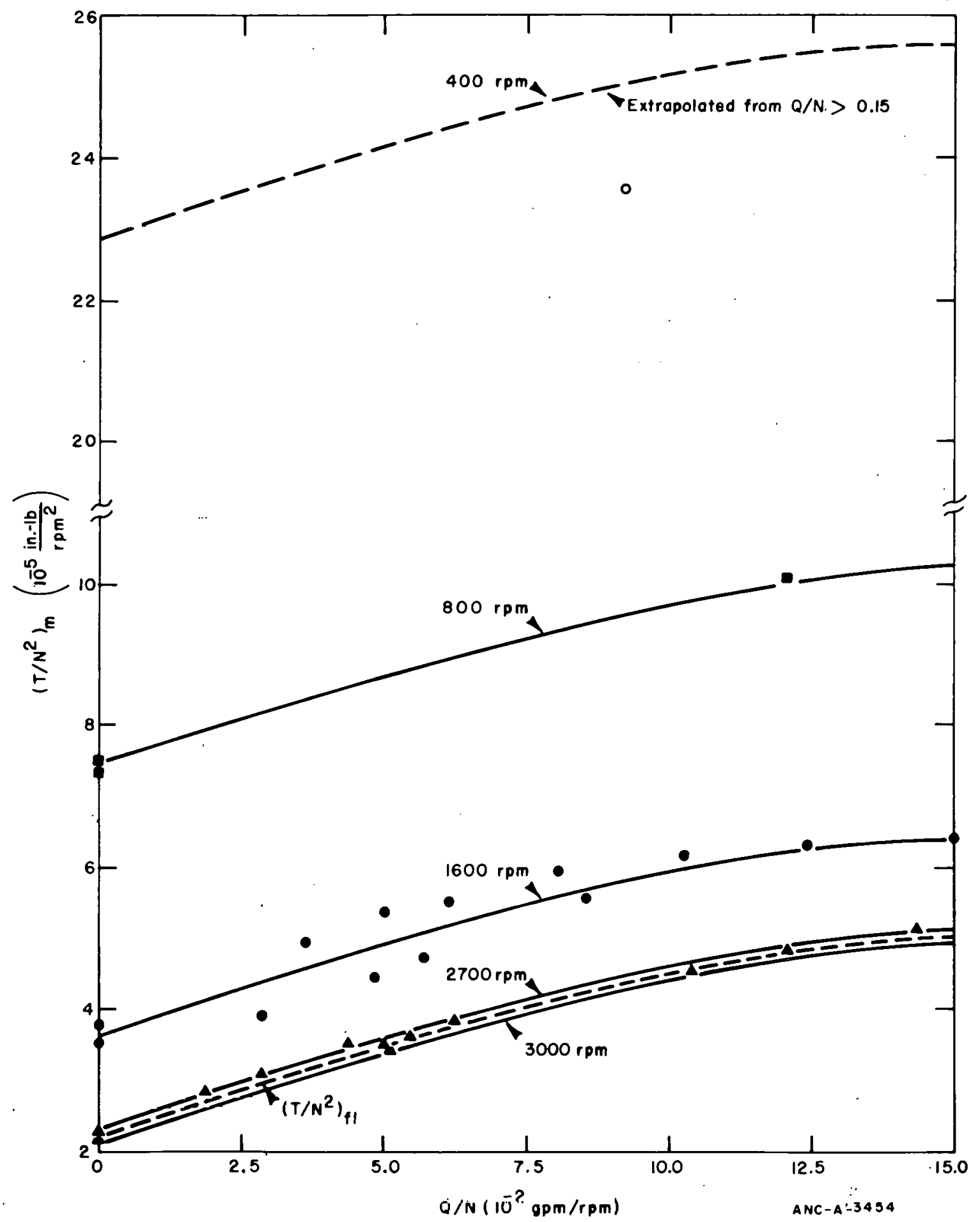

Fig. 9 Single-phase normal torque characteristics. 
by the impeller speed squared $\left(\mathrm{T} / \mathrm{N}^{2}\right)$ is plotted versus the capacity divided by the speed $(\mathrm{Q} / \mathrm{N})$. For a pump adhering to the affinity. laws, with negligible frictional effects, the results for all speeds should result in a single curve. Little difference is noted between the results for 2700 and $3000 \mathrm{rpm}$ (about $7 \mathrm{in} .-\mathrm{lb}$ ); however, the variation appears quite significant as the speed is reduced. On the basis of the slight change between the results for the speeds of 2.700 to $3000 \mathrm{rpm}$, torque chargeable to hydraulic losses was assumed to be approximatcd by the average of the results from the 2700- and 3000-rpm cases: The frictional term 'was. then, found from the following relation:

$$
\left(\mathrm{T}_{i} / \mathrm{N}_{i}^{2}\right)_{\mathrm{m}}=\left(\mathrm{T}_{j} / \mathrm{N}_{j}^{2}\right)_{\mathrm{f} 1 \mathrm{l}}+\left(\mathrm{I}_{i} / \mathrm{N}_{i}^{2}\right)_{\mathrm{fr}}
$$

where the subscripts refer to:

$$
\begin{aligned}
& \mathrm{i}, \mathrm{j}=\text { pump speed, } \mathrm{j}=2700 \mathrm{rpm} \\
& \mathrm{fl}=\text { hydraulic torque } \\
& \mathrm{m}=\text { measured torque } \\
& \mathrm{fr}=\text { frictional torque. }
\end{aligned}
$$

Rearrangement: of Equation (1) results in:

$$
\left(T_{i} / N_{i}^{2}\right)_{f r}=\left(T_{i} / N_{i}^{2}\right)_{m} \cdot-\left(T_{j} / N_{j}^{2}\right)_{f l}
$$

or

$$
\left(T_{i}\right)_{f r}=\left[\left(T_{i} / N_{i}^{2}\right)_{m}-\left(T_{j} / N_{j}^{2}\right)_{f 1}\right] N_{i}^{2}
$$

The results of these calculations indicate that a constant average value of $33 \mathrm{in.}-1 \mathrm{~b}$ is appropriate for use as a correction term..This correction. value compares favorably with the average value of $38.8 \mathrm{in.-1b}$ obtained from a series of simplified measurements taken prior to the single-phase flow tests by applying a steady pull to the pump shaft. The fact that the two methods of approximating frictional torque result in values of the same order of magnitude indicates that the application of a constant correction is valid. Since the value of 33 in.-lb results from use of a broader data base, this value was used as the correction factor. The results of replotting the corrected data as a homologous curve are shown in Figure 10; the scatter exhibited in Figure 7 is significantly reduced by the method discussed. The four-quadrant torque characteristic curves, modified to account for.frictional torque, are shown in Figure 11. 


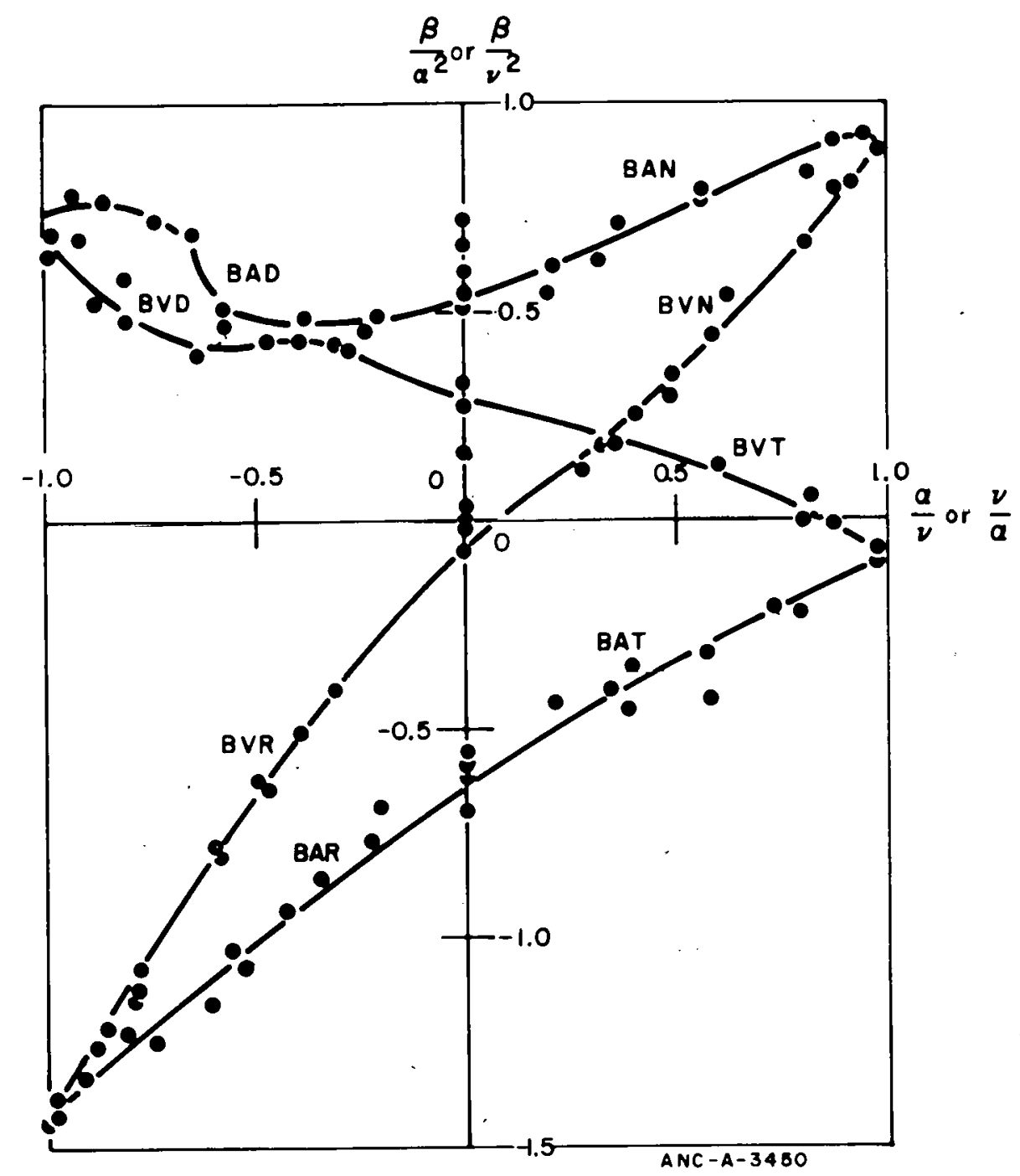

Fig. 10 Single-phase homologous torque curve for the Mod-1 semiscale pump (corrected for frictional torque).

\subsection{Loss Characteristics}

In the study of the LOCA, two pump loss characteristics can significantly influence system response: (a) stopped rotor forward flow resistance and (b) stopped rotor reverse flow resistance. Factors contributing to these hydraulic losses in a centrifugal pump include skin friction, eddy and separation losses, and diffusion losses. Analysis of the contribution of each factor is particularly complex because at no point in the path from the suction to the discharge nozzle is either the area or shape of the flow path or the direction of flow constant. Rotation of the impeller increases the complexity of the velocity distribution. In general, even the combined effect of each of the factors contributing to the losses cannot be quantified accurately. A simplified approach is to assume that all of the losses in a 


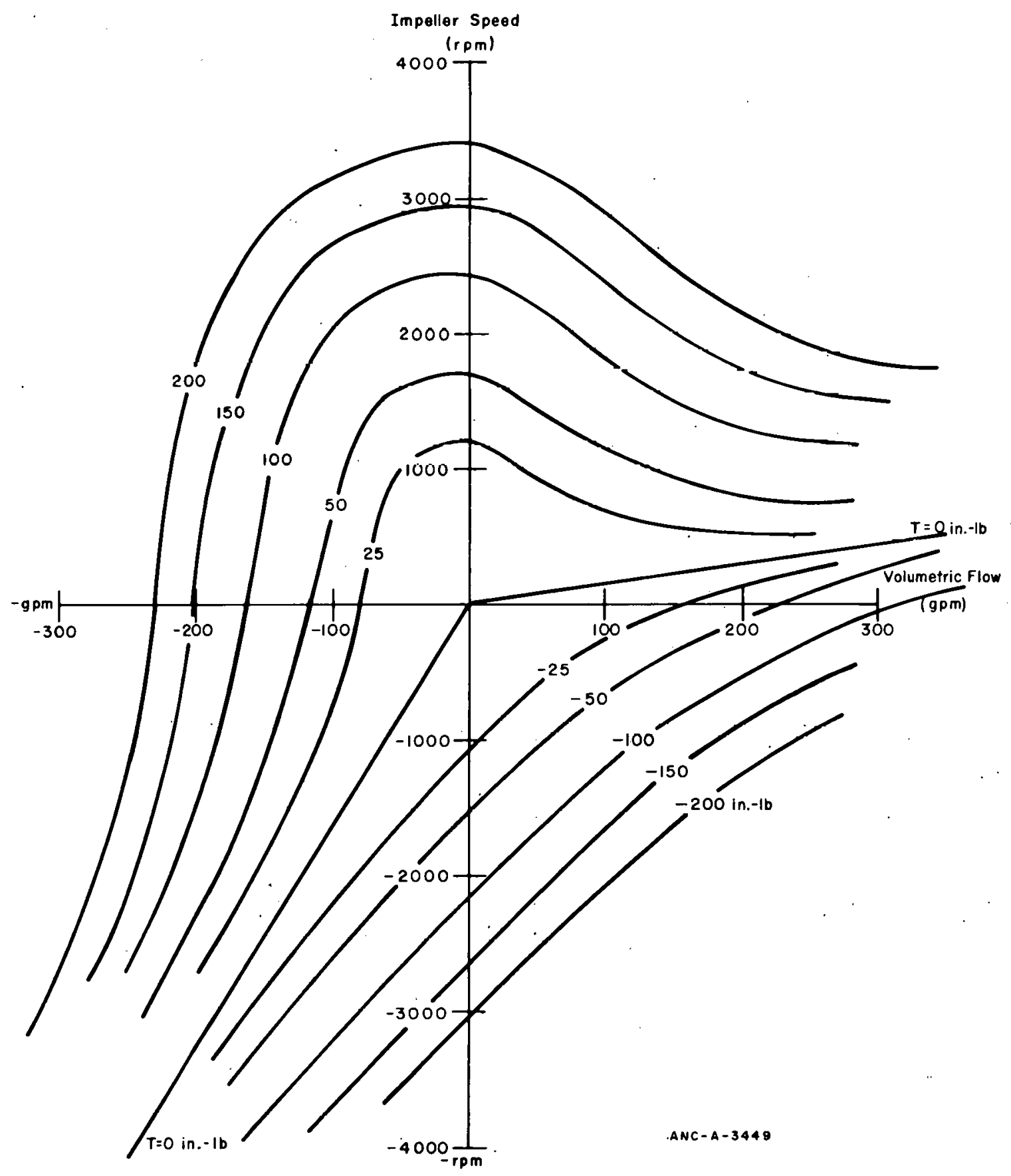

Fig. 11 Single-phase four-quadrant torque curves for the Mod:1 semiscale pump.

centrifugal pump can be lumped into one term. The information contained in the homologous curves can be translated into a commonly used lumped resistance parameter by the following approach:

Darcy's equation relating head, hydraulic losses, and fluid velocity is:

$$
H=f \frac{\ell}{D} \frac{v^{2}}{2 g}
$$


where

$$
\begin{aligned}
\mathrm{H} & =\text { static head, } \mathrm{ft} \\
\mathrm{f} & =\text { Darcy friction factor, dimensionless } \\
\frac{\ell}{\mathrm{D}} & =\text { length-to-diameter ratio, dimensionless } \\
\mathrm{V} & =\text { fluid velocity, } \mathrm{ft} / \mathrm{sec} \\
\mathrm{g} & =\text { gravitational constant, } \mathrm{ft} / \mathrm{sec} .
\end{aligned}
$$

By substituting an assumed overall dimensionless resistance coefficient,

$$
\mathrm{K}^{*}=\mathrm{f} \frac{\ell}{\mathrm{D}}
$$

which incorporates all of the complex hydraulic losses within the pump volute together with entrance and exit losses, into Equation (4) the following expression is obtained relating head loss to resistance coefficient:

$$
\mathrm{H}=\mathrm{K} * \frac{\mathrm{V}^{2}}{2 \mathrm{~g}}
$$

Since the volumetric flow, $\mathrm{Q}\left(\mathrm{ft}^{3} / \mathrm{sec}\right)$, is the product of the fluid velocity, $V(\mathrm{ft} / \mathrm{sec})$, and the flow area, $A\left(\mathrm{ft}^{2}\right)$, substitution of $\mathrm{Q}^{2} / \mathrm{A}^{2}$ into Equation (6) for $\mathrm{V}^{2}$ results in

$$
\mathrm{H}=\mathrm{K} * \frac{\mathrm{Q}^{2}}{2 \mathrm{gA}^{2}}
$$

or

$$
\mathrm{K}^{*}=\frac{2 \mathrm{gA}^{2} \mathrm{H}}{\mathrm{Q}^{2}} \text {. }
$$
produces

Substitution of the homologous definitions, $\mathrm{h}=\mathrm{H} / \mathrm{H}_{\mathrm{r}}$ and $v=\mathrm{Q} / \mathrm{Q}_{\mathrm{r}}$, into Equation (8)

$$
\mathrm{K}^{*}=\frac{\mathrm{h}}{\nu^{2}}\left[\frac{2 \mathrm{gA}^{2} \mathrm{H}_{\mathrm{r}}}{\mathrm{Q}_{\mathrm{r}}{ }^{2}}\right]
$$

A resistance coefficient, $R^{\prime}$, which relates measurable variables is defined as

$$
R^{\prime}-\frac{\Delta P \rho}{w^{2}}
$$


where

$$
\begin{aligned}
\mathrm{R}^{\prime} & =\text { resistance coefficient, } 1 \mathrm{~b}_{\mathrm{f}}-\mathrm{sec}^{2} / \mathrm{lb}_{\mathrm{m}}-\mathrm{in} .^{2}-\mathrm{ft}^{3} \\
\Delta \mathrm{P} & =\text { pressure drop, } 1 \mathrm{~b}_{\mathrm{f}} / \mathrm{in} .^{2} \\
\rho & =\text { fluid density, }, \mathrm{bb}_{\mathrm{m}} / \mathrm{ft}^{3} \\
\mathrm{w} & =\text { flow rate, } \mathrm{lb}_{\mathrm{m}} / \mathrm{sec} .
\end{aligned}
$$

Since $w=\rho Q$ and $H=(144) \triangle \mathrm{P} / \rho$, Equation (10) can be rewritten as

$$
H=144 Q^{2} R^{\prime}
$$

Equating Equations (7) and (11) results in

$$
R^{\prime}=\frac{K^{*}}{2(144) g A^{2}}
$$

Substitution of Equation (9) into Equation (12) gives

$$
R^{\prime}=\frac{h}{\nu^{2}}\left(\frac{H_{r}}{144 Q_{r}{ }^{2}}\right) \text {. }
$$

The quantity in parentheses in Equation (13) is constant for a given pump ( 8.33 for the Mod-1 pump). The maximum value of $R^{\prime}$, therefore, depends on the maximum absolute value of $\mathrm{h} / v^{2}$ which can be obtained from the homologous curves developed from the single phase data. The homologous head curve presented earlier for the Mod-1 pump is reproduced as Figure 12. Point A represents the point of maximum forward-flow stopped rotor resistance for the subject pump, and the value of the resistance in terms of the resistance coefficient, $\mathrm{R}^{\prime}$, is $2.9 \frac{\mathrm{b}_{\mathrm{f}}-\mathrm{sec}^{2}}{\mathrm{~b}_{\mathrm{m}}-\mathrm{ft}^{3}-1 \mathrm{n}^{2}}$. During certain LOCA simulations, the resistance of the pump in the intact loop of the semiscale system approaches this maximum as the decompression process accelerates fluid in the normal direction through the pump beyond its capacity to pump. For this situation, pump operation is along the HVN curve from initial conditions, $\mathrm{X}$, to maximum resistance, A. Figure 13 shows the relationship between resistance and impeller speed for the Mod-1 pump for forward flow. As indicated, the resistance increases rapidly with increased flow and exponentially approaches the maximum value.

Equation (13) and the homologous curve presented as Figure 12 can also be used to obtain the reverse flow resistance. Point B on Figure 12 represents the value of reverse-flow stopped rotor resistance $\left(6.0 \frac{b_{f}-s^{2} c^{2}}{l_{m^{-}} \mathrm{ft}^{3}-i n .^{2}}\right.$ for the subject pump) and rather than being a 


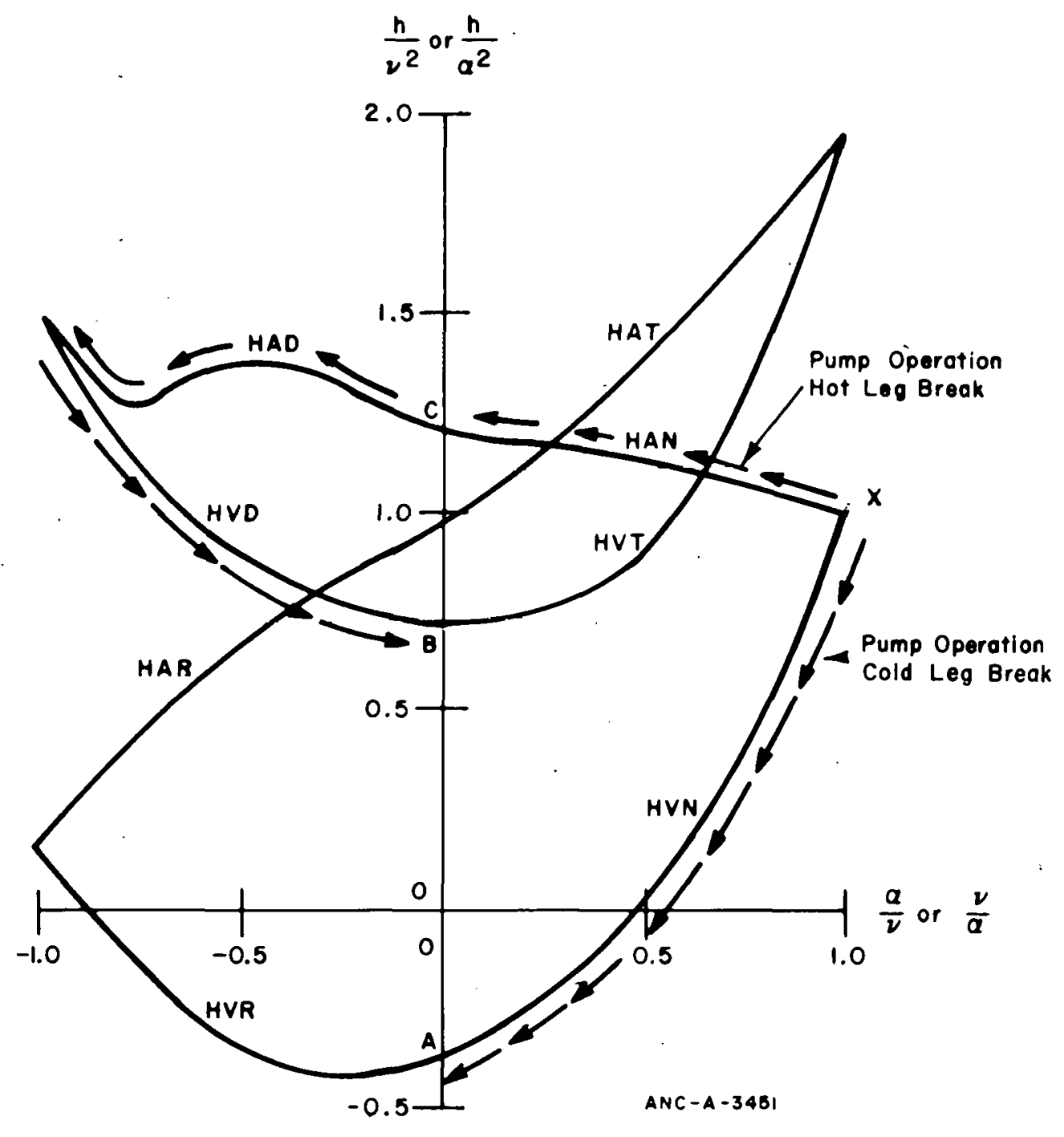

Fig. 12 Pump operation and losses with reference to homologous head curve.

maximum it is a minimum resistance for reverse flow. The pump in the intact loop of the semiscale system would approach this point from initial conditions, $X$ along the HAN curve, reaching shutoff head, (infinite resistance) at Point C. From Point $C$, the pump would enter the energy dissipation region (HAD, HVD) where the impeller rotating in the forward direction would tend to oppose the now reverse flow of fluid, thereby resulting in an increased hydraulic resistance. Eventually the fluid flowing in the reverse direction would slow the impeller, reducing the hydraulic resistance, along the HVD path until at Point B impeller direction would reverse and the zone of turbine operation would be reached. Curves based on single-phase flow data of resistance versus reverse flow rate as a function of impeller speed for the Mod-1 pump are shown in Figure 14. As indicated, the resistance drops rapidly from infinity (shutoff head) and approaches the minimum value of stopped rotor resistance at high negative flows. 


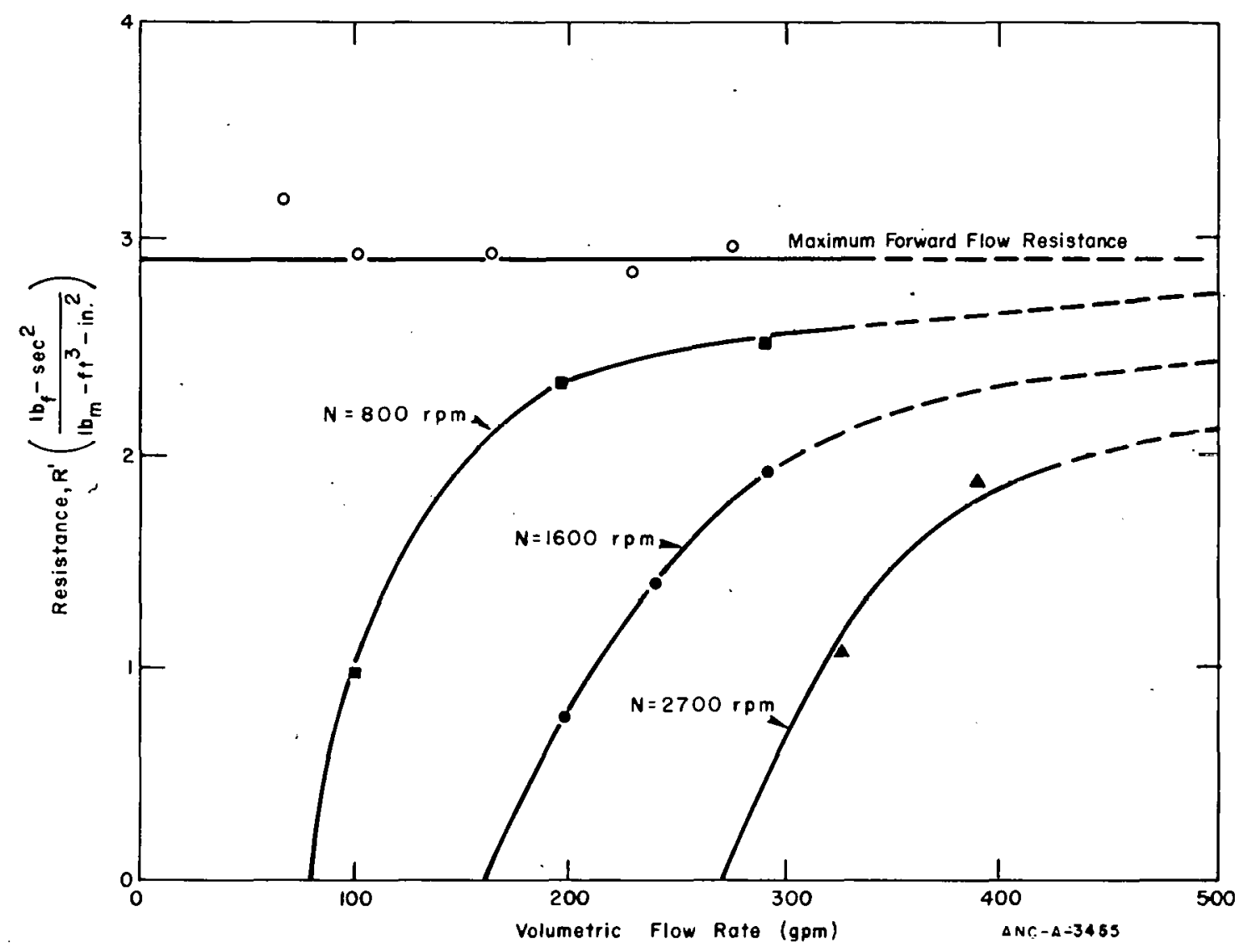

Fig. 13 Forward flow resistance for the Mod-1 pump.

\section{STEADY STATE TWO-PHASE PERFORMANCE CHARACTERISTICS}

The basic approach to the analysis of the steady state two-phase performance data obtained for the Mod-1 semiscale pump was to use the single-phase performance curves developed in the previous section, both the homologous and the more tractable normalized pump characteristic curves, to evaluate the influence of void fraction.

Since the net pressure rise across a pump is dependent on both the density of the fluid in the impeller and the head losses through the pump, neither of which can be directly measured or calculated with any accuracy, evaluation of two-phase pump performance on the basis of suction or discharge conditions or some intermediate value is a matter of individual preference at least until such time as a particular set of conditions empirically has been established as best. The analysis reported in this report is based on pump inlet fluid conditions.

\subsection{Two-Phase Homologous Ratios}

The two-phase data points in Reference 3.were selected on the basis of the assumption that the pump performance under two-phase fluid conditions could be related to the 


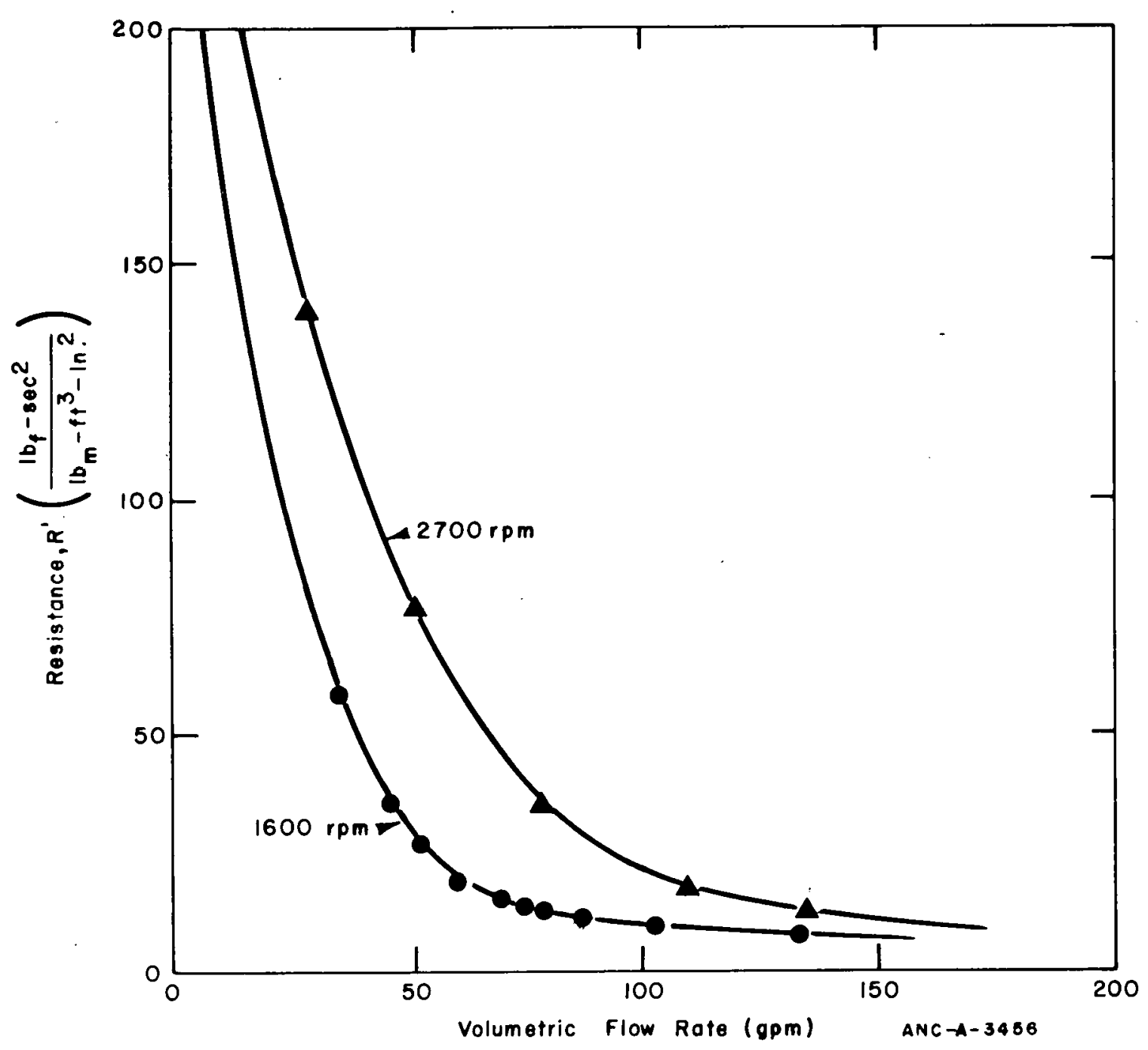

Fig. 14 Reverse flow resistance for the Mod-1 pump.

single-phase pump performance through the use of homologous ratios. This approach provided a means of evaluating the validity of a commonly used analysis assumption (that is, the assumption that single-phase homologous curves could be used as a first order approximation to the pump performance during the transient two-phase conditions of blowdown). The two-phase data points from Reference 3 are compared in Figure 15 with the empirical single-phase homologous curve. Wide scatter in the data is apparent in all regions of pump operations investigated. Attempts to separate data points in terms of inlet, outlet, or average void fraction to determine possible trends as functions of void fraction were unproductive for void fractions in excess of about $20 \%$. For void fractions less than $20 \%$, the single-phase characteristics were approximated fairly well, indicating that single-phase homologous characteristics could be used to approximate the behavior of the semiscale pump for inlet fluid conditions up to $20 \%$ void fraction. 


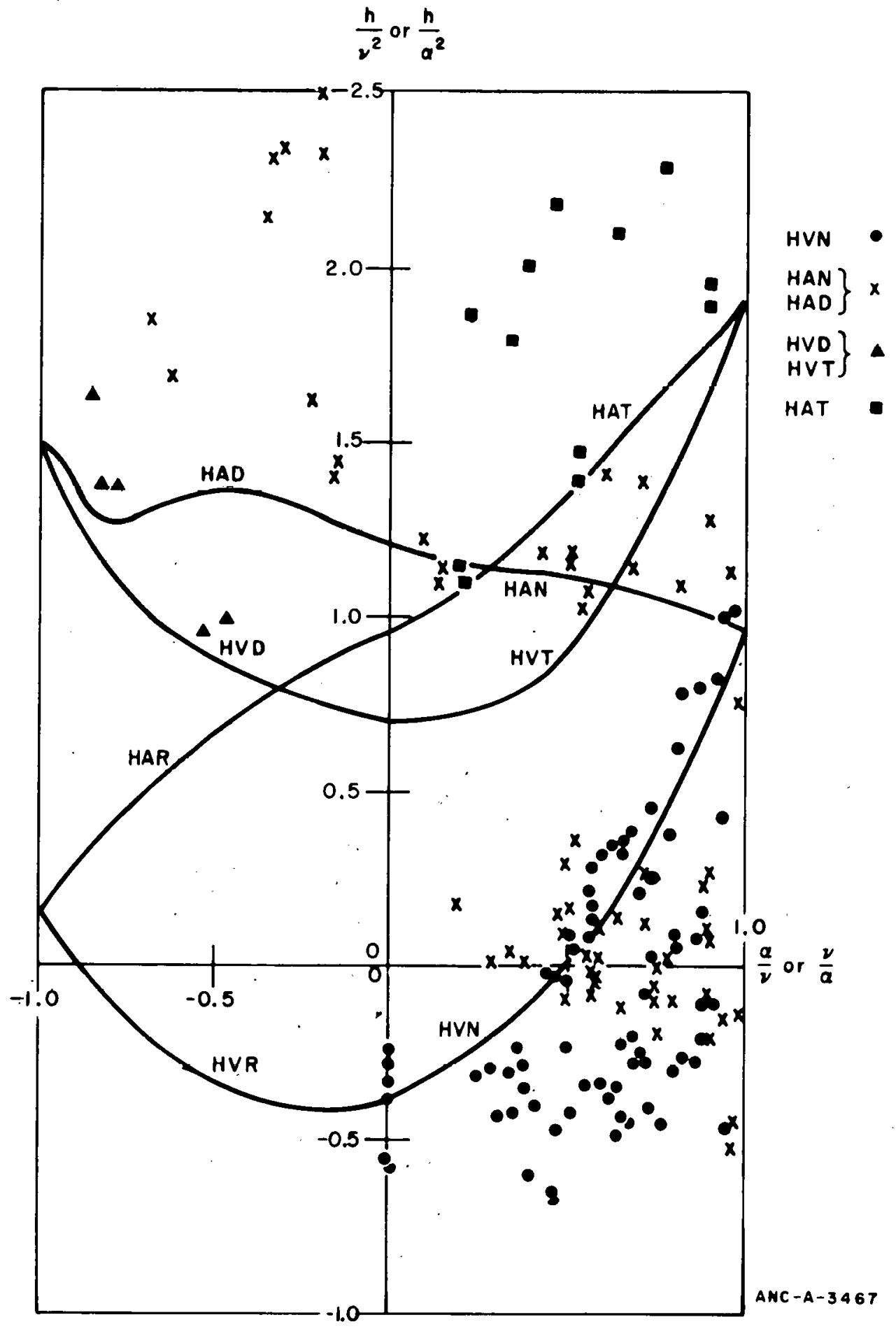

Fig. 15 Two-phase homologous ratios.

\subsection{Normalized Pump Characteristics - Head Performance}

To minimize the scatter due to void fraction in the two-phase data in the normal pump operating mode (HVN curve segment of Figure 15), the data are plotted as $\mathrm{H} / \mathrm{N}^{2}$ 
versus $\mathrm{Q} / \mathrm{N}$ as a function of four separate void fraction ranges in Figures 16 through 19. The solid line in all figures represents the results of the single-phase analysis. The data presented on Figure 16 indicate that most of the two-phase data obtained at void fractions below $25 \%$ reflect essentially the same pump performance as the single-phase (subcooled) data indicate. The points above the single-phase curve on Figure 16 (indicating two-phase performance better than single-phase performance) are due to the analysis being based on pump inlet fluid conditions. The apparent anomaly occurs because, for low inlet void fraction conditions, part of the head generated by the pump is used to collapse the steam voids and is not available for pumping. The discharge fluid is generally subcooled, or nearly so, such that basing pump head on inlet conditions results in the apparent head rise.

The difference in Figures 18 and 19 (void fractions from 50 to 100\%) between the two-phase data and the single-phase performance curve is particularly noticeable. The data points on Figures 18 and 19 are grouped together in the same region of the performance map with no apparent consistency or dependence on void fraction. This observation is inconsistent with expected pump behavior (that is, the pump would be expected to recover from full degradation and approach single-phase performance as the void fraction approaches $100 \%$ ). This discrepancy between single- and two-phase behavior of the pump strongly suggests that either the measurements are in error or are being improperly in terpreted.

Two-phase fluid conditions were obtained by separately metering the individual steam and water phases and then combining the two phases in a mixing device. This technique provides a reliable measurement of total mass flow rate into the pump. However, the connecting piping between the pump suction and mixer box was of significant length. A gamma attenuation device located immediately upstream of the pump suction provided a measurement of fluid density which was used in calculations of the average void fraction at the pump inlet. The single-beam gamma attenuation method of obtaining the fluid density during the two-phase tests tends to force the assumption of homogeneous flow at the measurement cross section. If the flow regime is other than homogeneous then the application of the measurement over the total pipe cross section would be in error due to the effects of pipe curvature. Since many of the data points were obtained with a mass velocity below $2 \times 10^{6} \mathrm{lb}_{\mathrm{m}} / \mathrm{sec}-\mathrm{ft}^{2}$ (generally considered to be the lower limit for steady state, homogeneous flow in horizontal runs), a likely source of error is introduced into the interpretation of the suction density by assuming homogeneous flow.

The potential error in the void fractions determined from the gamma attentuation measurement as a result of processing the data on the basis of a homogeneous assumption can be eliminated if the flow regime is known. Appendix B provides a procedure for correcting the output of the gamma measurement to obtain average density and void fraction for annular or completely stratified flow. The average density reported in Reference 3 was reevaluated point by point through use of the methods in Appendix B with the probable flow regime for each data point determined from a Baker flow regime map based on the measured mass flow rates and fluid states. This effort did not result in a significant improvement in the data correlation. 


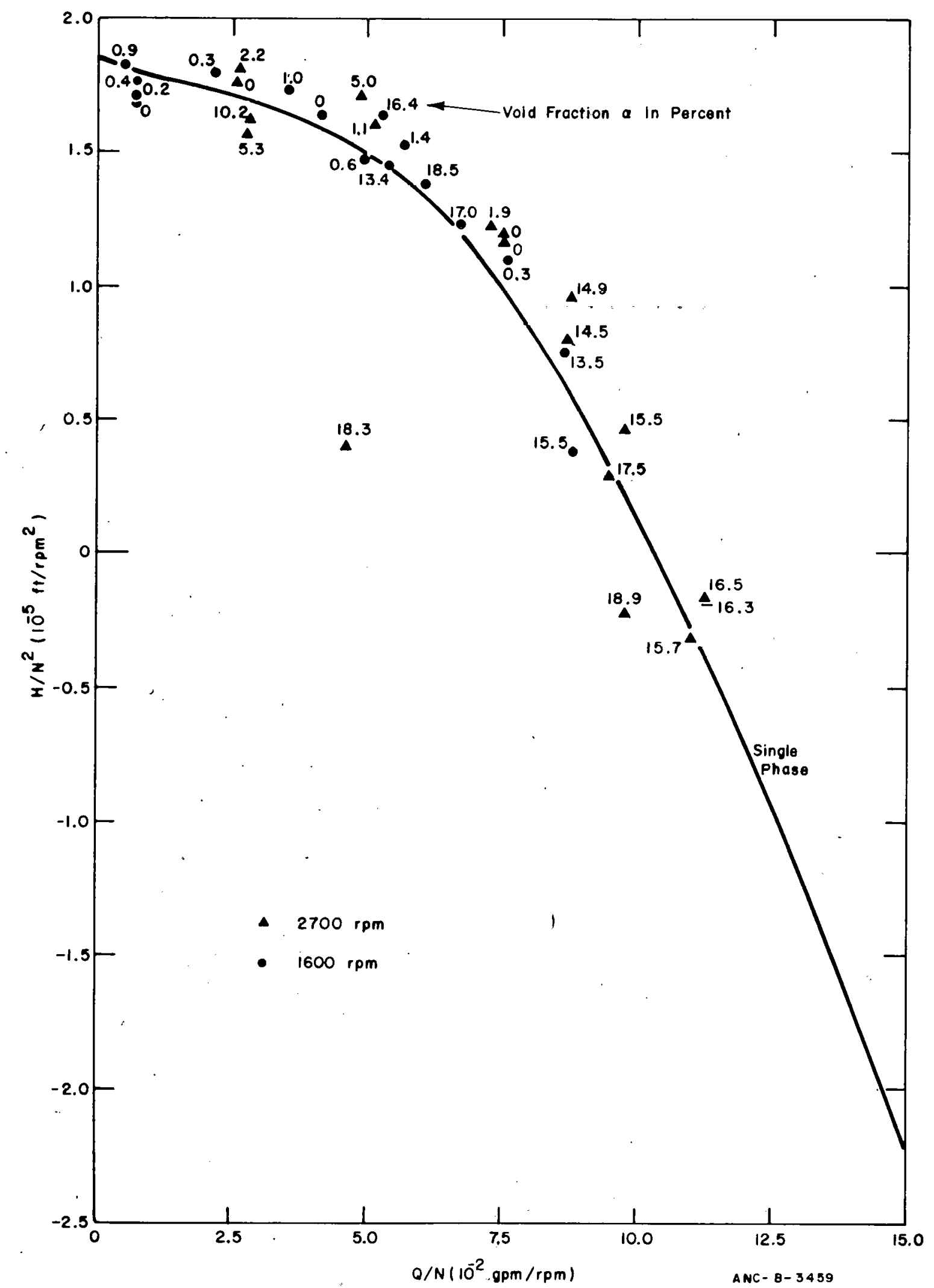

Fig. 16 Two-phase pump characteristics - $0 \leqslant \dot{\alpha} \leqslant 0.25$. 


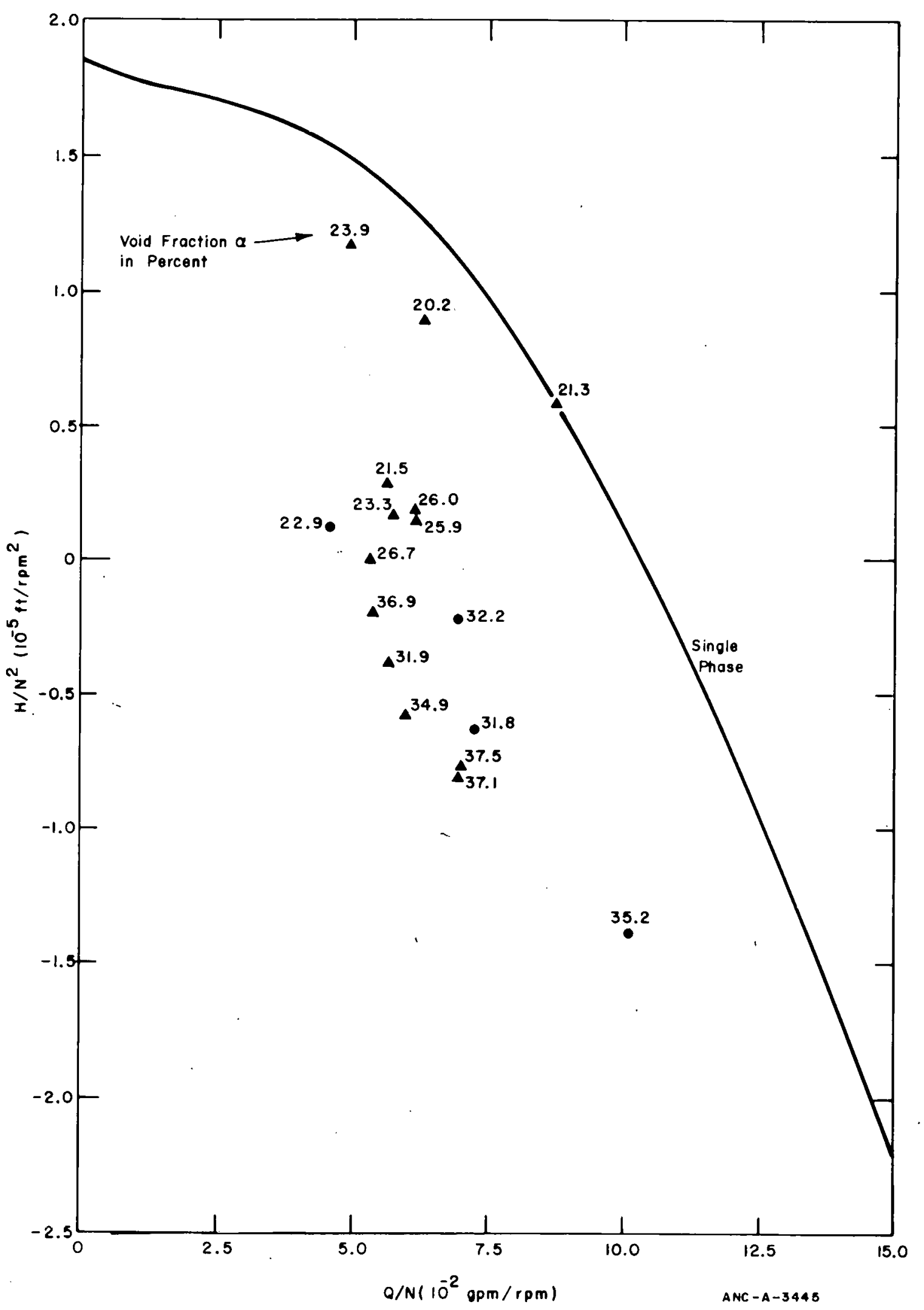

Fig. $17 \overline{7}$ Two-phase pump characteristics $-0.25 \leqslant \alpha \leqslant 0.50$. 


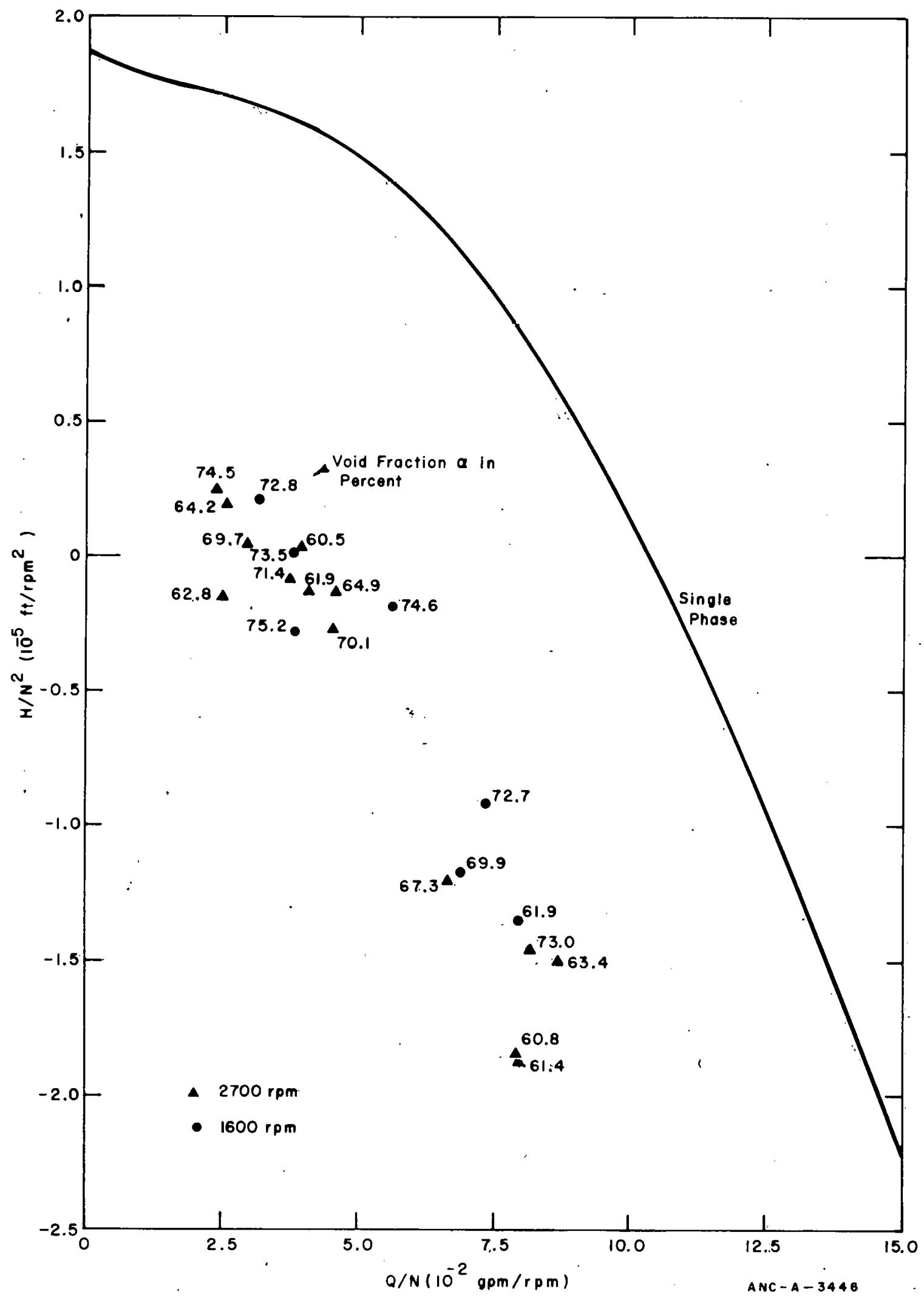

Fig. 18 Two-phase pump characteristics $-0.50 \leqslant \alpha \leqslant 0.75$. 


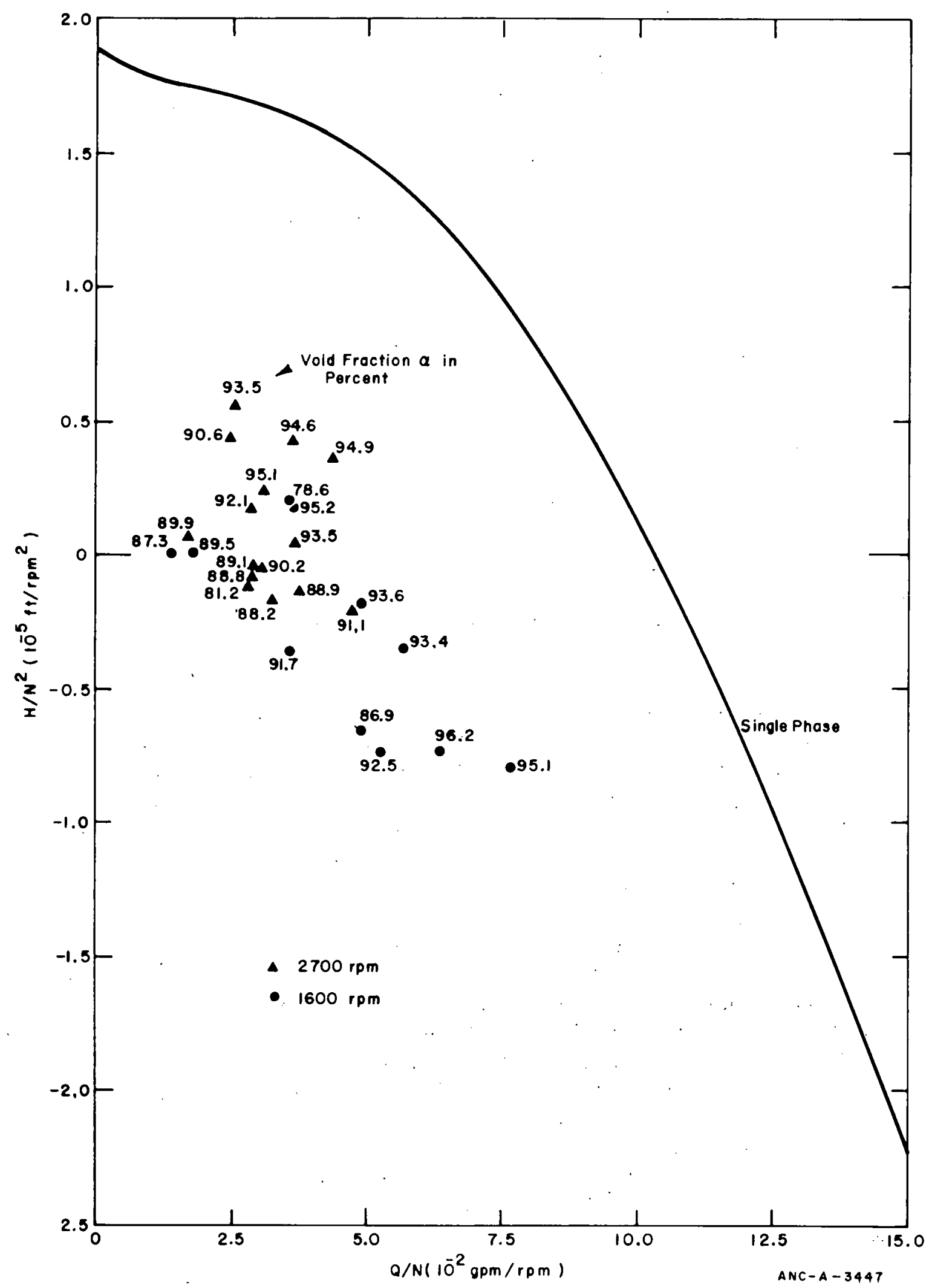

Fig. 19 Two-phase pump characteristics $-0.75 \leqslant \alpha \leqslant 1.0$. 
Under separated two-phase flow conditions, the suspected pump reaction is not to the local static density or void fraction measured upstream of the pump suction, but rather to the relative volumetric fluxes of the two phases entering the eye of the impeller. If the pump does indeed react to the volumetric flux, the actual static density obtained at the measurement locations:must be modified to account for the relative velocities (slip ratio) of the two phases. Appendix B also presents a method developed to define an "effective density" at the pump impeller. This effective density is a function of the upstream local static void fraction and slip ratio as determined from the flow regime map presented by Govier and Omer ${ }^{[14]}$. The basic assumption in this analysis is that the two phases are mixed homogeneously as they enter the pump cavity. The data presented previously in Figures 16 through 19 are replotted in Figures 20 and 21 on the basis of the effective density rather than the density obtained directly from the gamma attenuation system. The results, particularly those in Figure 21 which represents the data previously presented in Figures 18 and 19, indicate that the method used in this analysis does in fact separate the data into approximate void fraction regions.

Error bars have also been included in Figures 20 and 21 , for those cases in which an oscillating differential pressure was observed across the pump. The absolute value of the oscillation was generally small relative to the'system pressure; however, in terms of feet of inlet head, the variation is large, as indicated for some high void fraction points in Figure 21. For points without bars the pressure differential was constant. Whether the observed variations are internal to the pump or due to system oscillations cannot be determined because the variation in differential pressure is generally very small compared to the range of the system pressure transducers.

The available data are inadequate for identifying definite curves of head or performance degradation as functions of void fraction. Definition of pump behavior for cases in which the performance changes drastically with little change in inlet void fraction is the primary difficulty. For example, at.a $\mathrm{Q} / \mathrm{N}$ of about 0.06 , Figure 20 shows that the head degradation increases from 30 to $85 \%$ as the void fraction increases from $19.51021 \%$. The data at best support the construction of approximate limits of performance degradation in terms of void fraction ranges. These approximlate limits have becn defined and are compared on Figures 22 and 23 with the single-phase performance curves. The limits of the void fraction regions defined by data are included as a solid line.

The shape of the limit curves shown on Figures 22 and 23 are somewhat different than would be expected based on the basis of other pump tests. Sozzi and Burnette ${ }^{[2]}$ observed that at low flows the degradation for a given void fraction (maximum about $40 \%$ ) was less significant than at high flows. At low flow conditions, the performance curves approach the single-phase curve rather than becoming parallel to the single-phase curve as depicted in Figures 22 and 23. This oobservation is reasonable (at relatively low void fractions for which the pump is not completely degraded) for a high head pump with an upward sloping performance curve because more head is available at low flows to compress the voids.

The results on Figure 22 indicate that, for the Mod-1 pump, single-phase performance characteristics in the normal pump region are a good indicator of two-phase performance up 


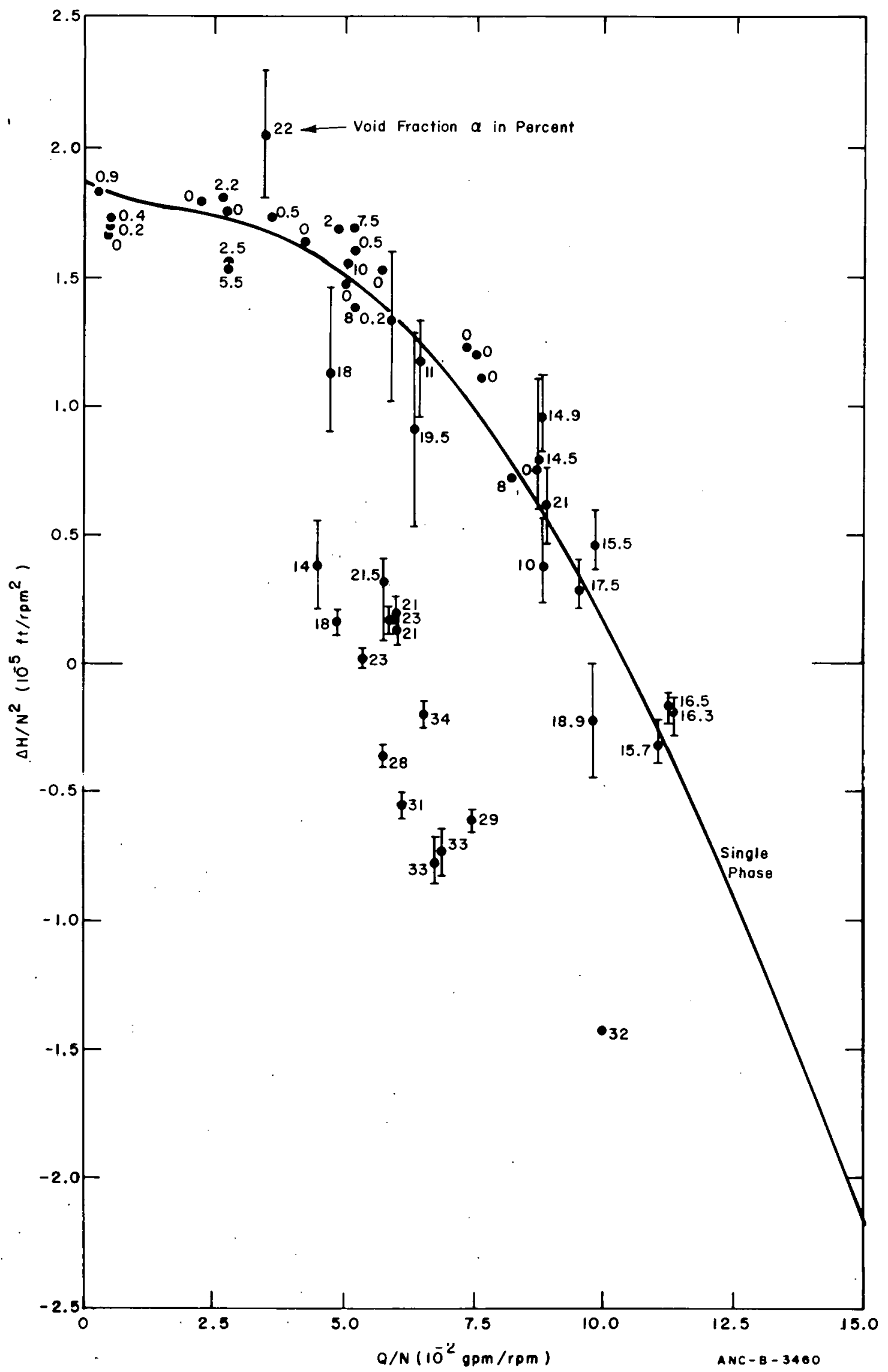

Fig. 20 Two-phase pump characteristic corrected for flow regime effects $-0 \leqslant \alpha \leqslant 0.50$. 


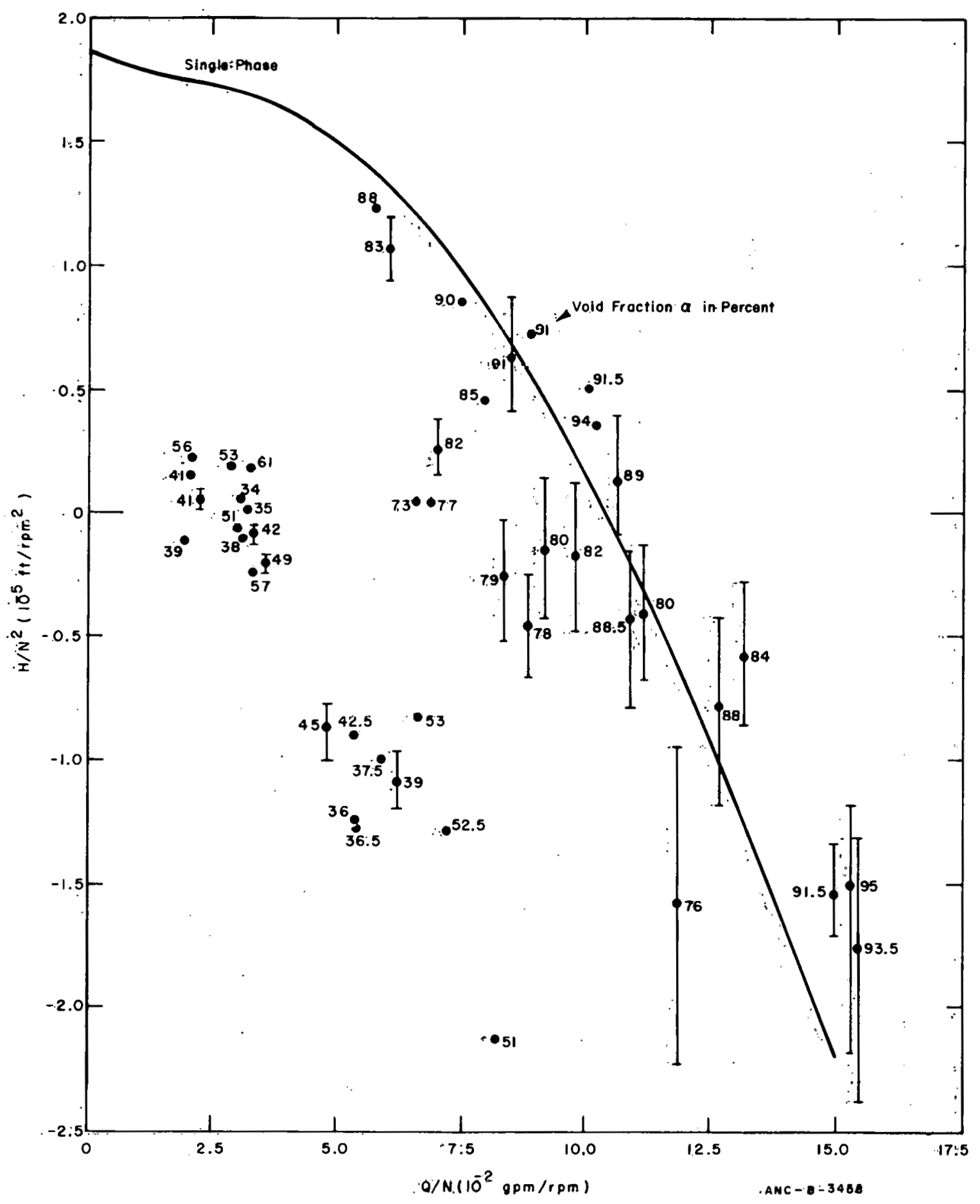

Fig.:2l Two-phase pump characteristics corrected for'flow regime.éffects $-0.50 \leqslant \alpha: \leqslant: 1.0$.

to inlet void fractions of about $18 \%$. Loss of performance occurs rapidly at void fractions greater than about $18 \%$, with significant degradation occurring in the void fraction region from 18 to $24 \%$. Recovery in performance occurs at void fractions greater than $40 \%$ with the maximum value of degradation relatively insensitive to void fraction in the range of 30 to $6.0 \%$. 


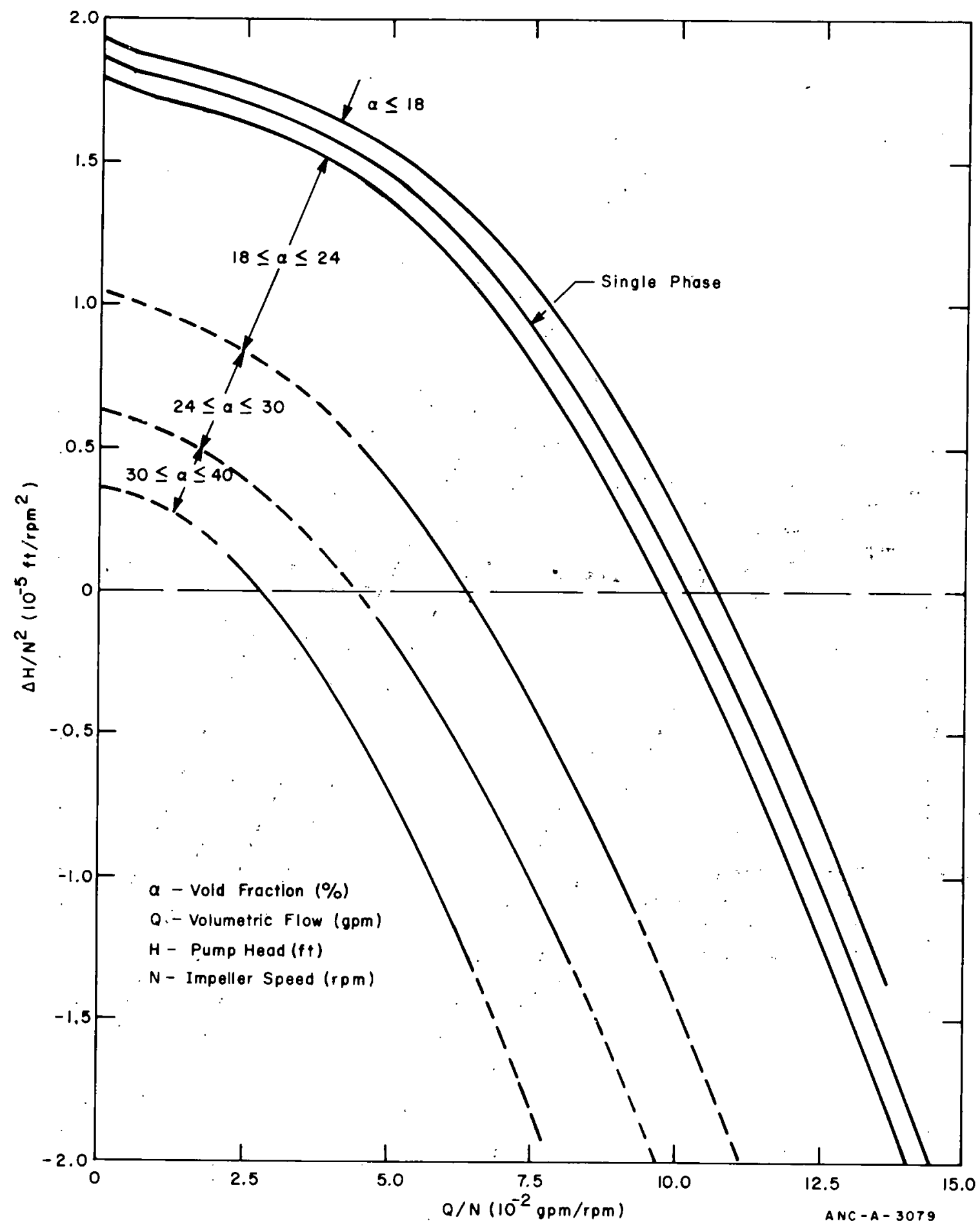

Fig. 22 Stcady state two-phase degradation performance as a function of inlet void fraction.

For the higher void fractions $(>85 \%)$ recovery performance is less well defined. The data points resulting from using an effective density are placed too high and too far to the right in comparison with the single-phase results. Since the effective density was calculated on the basis of a void fraction resulting from an assumption of pure annular flow, its value approaches that of saturated vapor in the high void tests because the physical situation is 


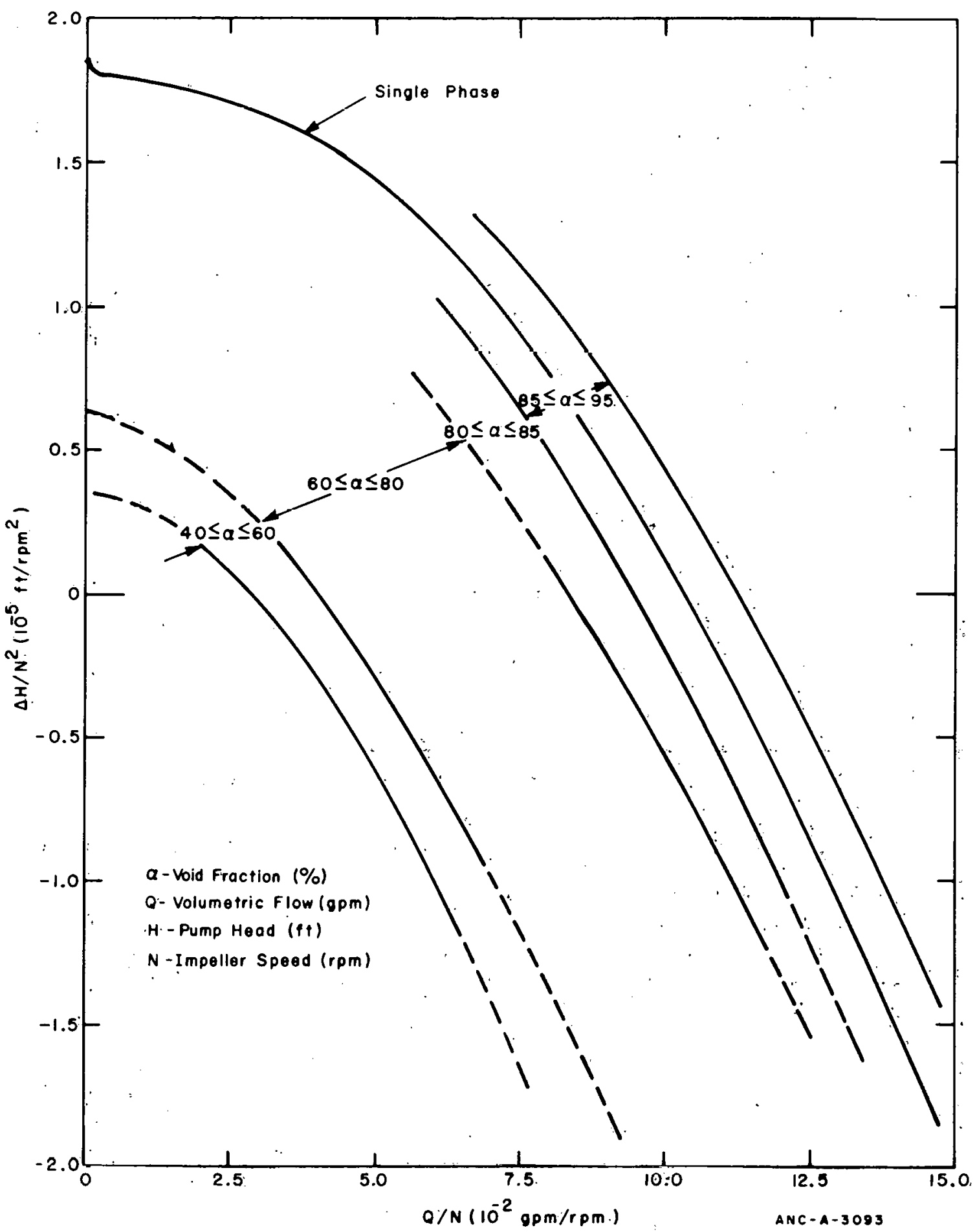

Fig. 23 Steady state two-phase recovery performance as a function of inlet void fraction.

one of a large diameter gas core within a thin annular liquid film. A reasonable alternative physical description is one of a thin annular film containing most of the liquid volume but with a gas core containing entrained droplets (annular mist). The latter physical description (if it could be mathematically defined) would result in a slightly higher effective density which in turn would result in the points in question being moved down and to the left: 
The procedure used to improve the data correlation and construct limit lines is only one of several possible methods of compensating for the effects of flow regime during the steady state tests. Several semiempirical two-phase correlations and flow regime maps are available in the literature which deal with predicting the volumetric concentration of a two-phase, flowing fluid as a function of the design and operating conditions. Generally, these correlations are developed for specific flow regime types. Hugmark and Pressburg [15] have developed an empirical holdup (or slip) correlation for vertical flow which is not dependent on exact knowledge of flow regime. In this correlation, the liquid holdup is plotted versus a holdup parameter that is a function of the physical properties of the fluid and the mass flow rate of the individual phases. A theoretical development of Zuber and

Findley ${ }^{[16]}$, also for vertical flow and independent of flow regime type, expresses average volumetric concentration in terms of the individual volumetric flow concentration of the phases and the drift velocity. Without knowledge of the volumetric flow rate of the phases, application of the available correlations involves essentially the same procedure used in the analysis presented herein. One fact that is apparent is that in order for future two-phase pump performance tests to produce meaningful data either the instrumentation state-ofthe-art must be advanced to permit measurement of the velocity and concentration profiles or provision must be made in the test loop to ensure homogeneous flow into the pump test section.

\section{TRANSIENT TWO-PHASE PERFORMANCE}

Transient tests were conducted as a part of the overall semiscale isothermal blowdown test program. In each of the blowdown tests the pump was operated at constant speed with the blowdown fluid flowing through the pump in the direction of normal pump operation. Typical data obtained from these tests relative to pump performance are included in Appendix A. In this section the transient two-phase head and torque performance is discussed relative to the single-phase steady state results, and the transient and steady state two-phase results are compared.

\subsection{Head Performance}

Figure 24 shows the typical response of the Mod-1 pump during the transient blowdown tests. The data are compared with the single-phase pump characteristic curve. The operating path during the transient is indicated and the inlet void fraction is provided for each data point. Increasing time into the blowdown transient is reflected by points of increasing void fraction as the system decompresses from subcooled liquid conditions to single-phase vapor conditions. The data approximate the results obtained from the steady state tests, particularly in the 0 to $60 \%$ void fraction range; however, in the high void fraction range the agreement is not as good.

The transient measurements are based on the same gamma attenuation density measuring technique (and the assumption of homogeneous flow) as used during the steady state tests; therefore, the possibility of flow regime effects is also present in the transient 


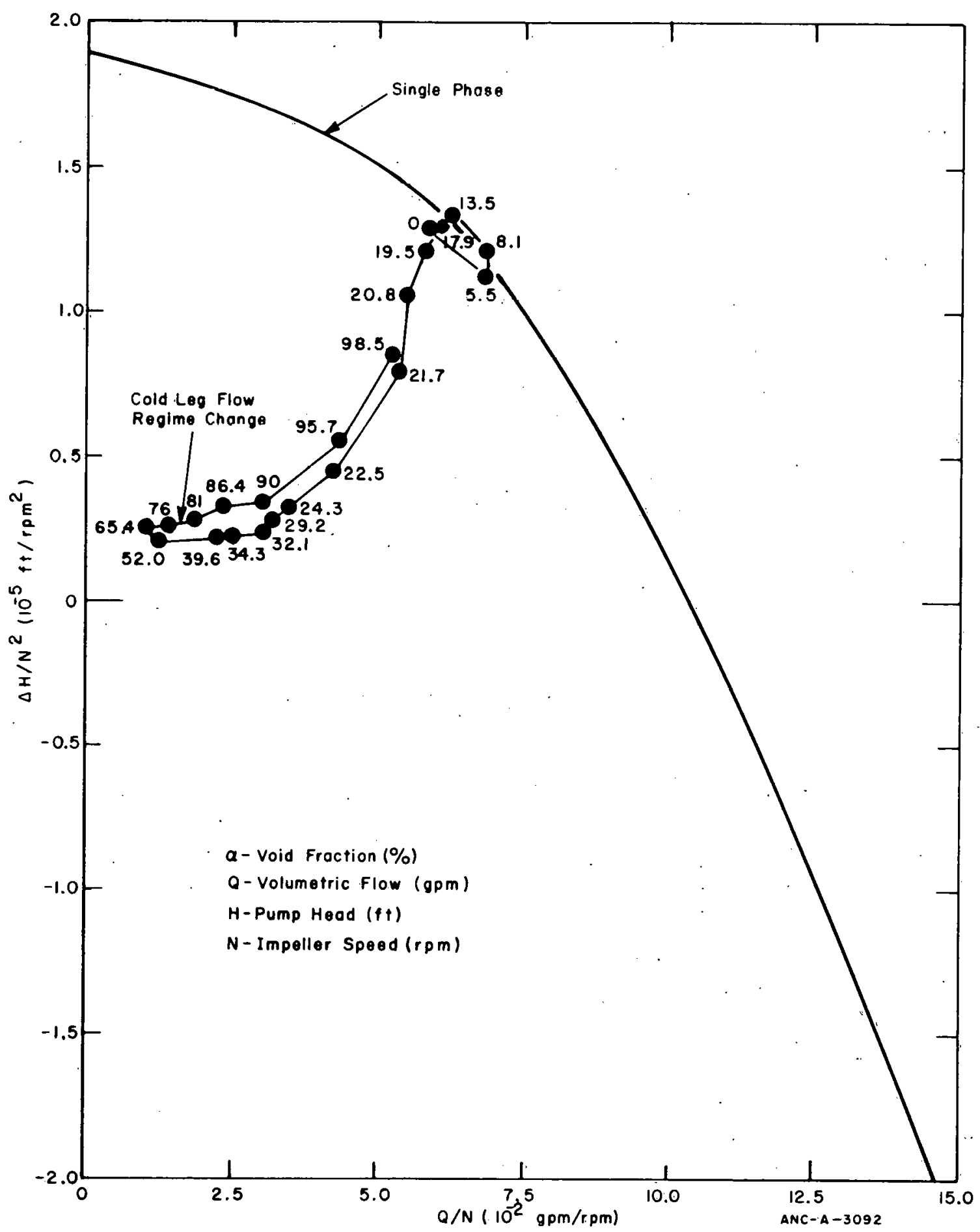

Fig. 24. Transient two-phase pump performance data -- Test 1009.

data. Measurements in other portions of the system indicate that homogeneous flow existed during the isothermal tests until about 8 to 10 seconds into the blowdown transient. At 10 seconds the system is essentially $80 \%$ voided of initial mass. Beyond this time, stratified flow is indicated in the operating loop. This point in time is indicated in Figure 24 by "cold leg flow regime change". Since no means is available for obtaining a primary 
measurement of mass flow rate during the blowdown transient, the method of obtaining an effective density for the steady state tests cannot be applied in the case of the transient data. An approximation can be made, however, by assuming stratified flow and calculating the density appropriate for stratified flow and then using that value in the calculation of pump performance rather than the value based on the homogeneous assumption. Figure 25 presents the data of Figure 24 together with the void fraction regions determined from the steady state two-phase results. As seen from Curve (a) the correspondence is very good for the first half of the void fraction range during which the performance is degrading. The transient results, like the steady state results, indicate that the pump performance can be approximated by the steady state characteristics up to void fractions of about $18 \%$. At void fractions greater than $18 \%$, loss of performance occurs rapidly, reaching a maximum at about 35 to $40 \%$ void fraction. Head degradation in the range from 30 to $60 \%$ is relatively independent of void fraction.

As was the case for the steady state results, recovery performance (Curve b) for the transient conditions is not well defined; however, use of the density calculated, assuming a stratified flow as a basis for calculating pump head and volumetric flow rate improves the results. These results (both steady state and transient) indicate that the influence of flow regime, particularly at high void fractions, is significant and that attempts to determine pump performance at high void fractions require definite knowledge of flow regime type and volumetric fluxes for the individual phases.

The agreement between steady state and transient behavior is supported by an analysis performed to determine whether the fluid inertial effects resulting from the rapid decompression of the blowdown tests would affect the transient performance. The results of that analysis are presented in detail in Appendix C. Briefly, the analysis indicated that inertial effects in the inlet and discharge lines to the pump were more significant than the inertial effects in the pump impeller. The magnitude of the fluid inertia in the lines, however, is insignificant when related to the generated head (less than $1 \%$ ).

\subsection{Torque Characteristics}

Typical transient torque chracteristics obtained from the isothermal test series are shown in Figure 26 and compared with the steady state single-phase results. The data have been corrected for frictional torque due to the bearing and seal losses as discussed in Section III-1.2. For all of the transient tests, the initial value of measured torque is higher than the results of the steady state tests would indicate for the flow rate and pump speed indicated. The discrepancy: has been found to be due to the increased bearing and seal friction torque caused by the higher initial system pressure of the blowdown tests. The approximate correction for frictional torque of $33 \mathrm{in.}-\mathrm{lb}$ was based on data taken at relatively low pressures (maximum 1000 psia). Results from the isothermal tests indicate that at 2250 psig the frictional torque is about 50 to $55 \mathrm{in} .-\mathrm{lb}$ and affirm that the value of $33 \mathrm{in} .-\mathrm{lb}$ is appropriate when system pressures decrease below about 1200 psig. With the exception of the initial performance value, the two-phase data indicate that the two-phase pump performance is approximated by the single-phase characteristics up to an inlet void fraction 

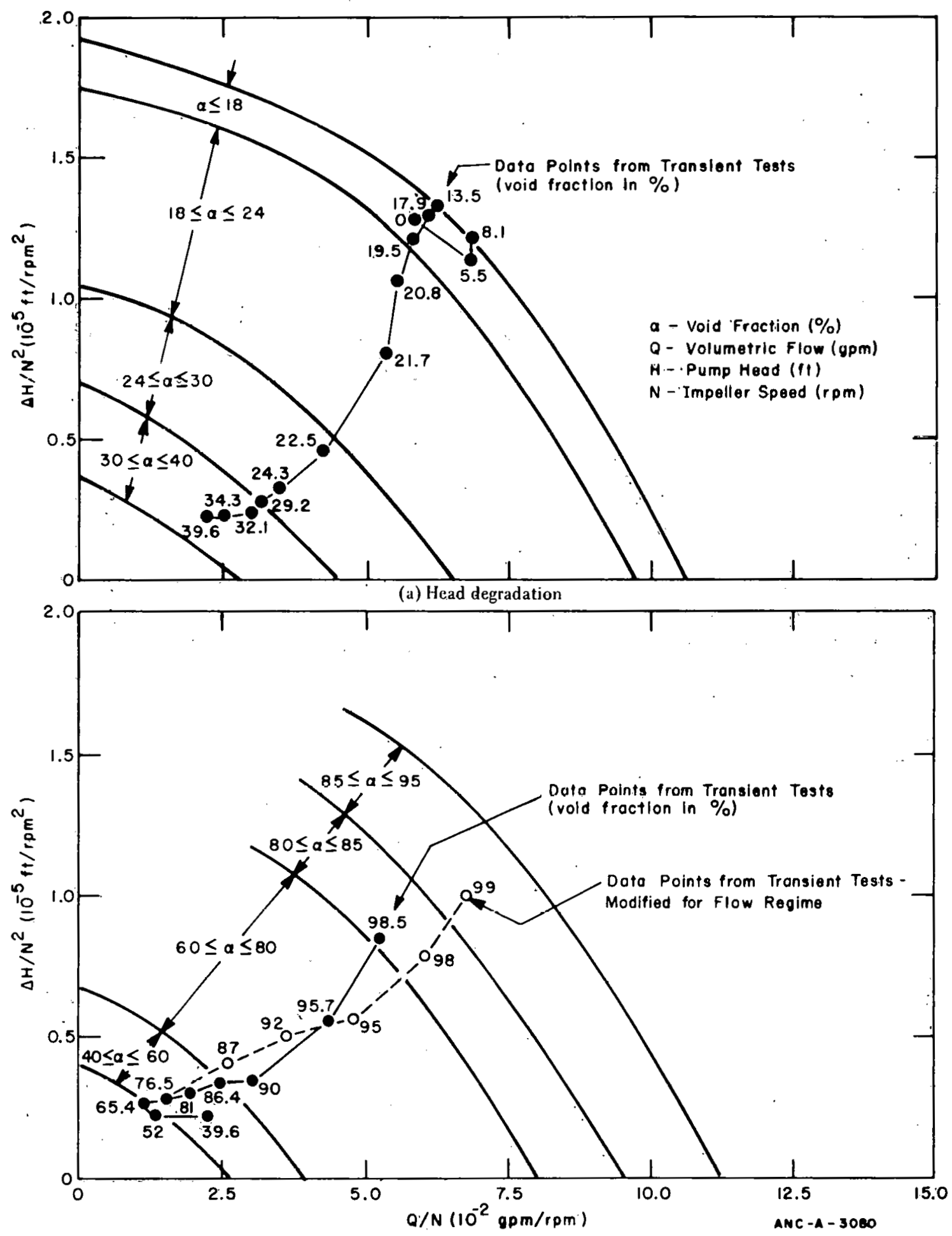

(b) Head recovery

Fig. 25 Comparison of steady state and transient two-phase pump performance.

of about $18 \%$. For void fractions greater than $18 \%$ the performance drops off rapidly, with maximum degradation resulting at about $40 \%$ void fraction. From about $40 \%$ void fraction the pump is in the recovery phase (more clearly defined by head performance, Figure 25); 


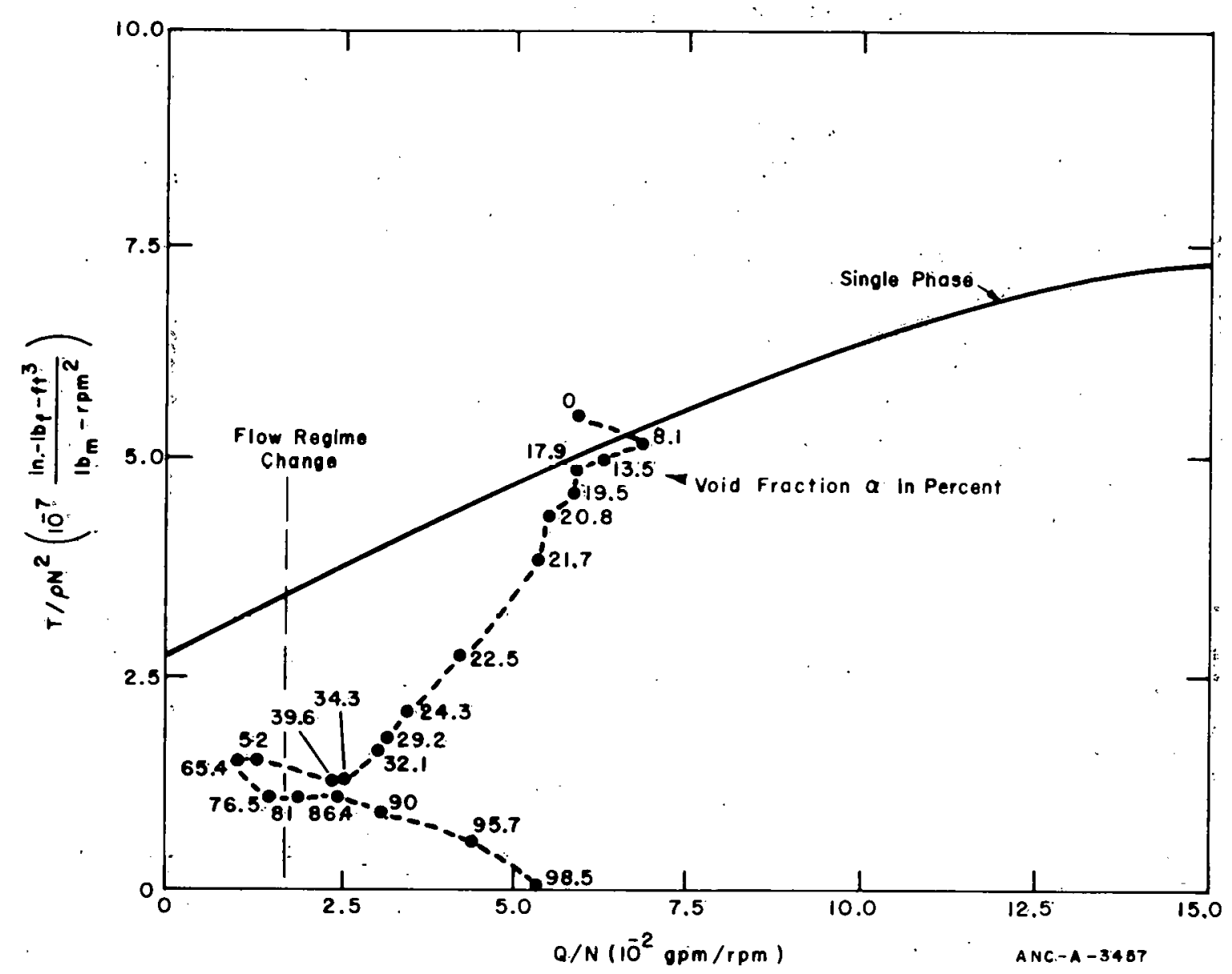

Fig. 26 Transient two-phase torque characteristics .- Test 1009.

however, the influence of the flow regime changes in combination with the low values of measured torque (same order of magnitude as frictional torque) prevents any definition of the trend of the data. 


\section{SUMMARY OF OBSERVATIONS AND CONCLUSIONS}

The single-phase steady state head and torque performance data follow the pump affinity relationships quite well. The measured torque values must be corrected by an average constant value of $33 \mathrm{in}$.lb to account for bearing and sed friction. The homologous head relationships are well defined by the steady state data taken, as are the homologous torque relationships when frictional torque is considered.

The forward and reverse stopped rotor resistances of the Mod-1 pump have been determined from the results of the single-phase homologous head relationships. The maximum stopped rotor forward flow resistance, $R^{\prime}$, for the Mod-1 pump is 2.9 $\mathrm{lb}_{\mathrm{f}}-\mathrm{sec}^{2} / \mathrm{lb}_{\mathrm{m}}-\mathrm{ft}^{3}-\mathrm{in} .^{2}$, and the stopped rotor reverse flow resistance is about 6.0 $\mathrm{lb}_{\mathrm{f}}-\sec ^{2} / \mathrm{lb}_{\mathrm{m}}-\mathrm{ft}^{3}-\mathrm{in} .^{2}$.

The two-phase data obtained from the steady state pump lests indicate that the method of expressing pump performance in terms of homologous ratios is appropriate for the Mod-1 pump at inlet void fractions up to $20 \%$.

Comparison of the steady state and transient two-phase results for the Mod-1 pump indicates similar behavior for the normal pump operating region. Under both steady state and transient conditions, the data indicate that performance degradation occurs in the range of 16 to $18 \%$ inlet void fraction. Loss of performance occurs rapidly at inlet void fractions greater than $18 \%$, particularly in the range from 18 to $24 \%$ inlet void fraction. For the transient results, maximum degradation occurs at about $35 \%$ void fraction. Recovery in performance occurs at void fractions greater than about $40 \%$ and performance degradation is relatively insensitive to inlet void fraction in the range of about 30 to $60 \%$. For the higher void fractions $(\alpha>80 \%)$ definition of performance is difficult. At the high void fractions, the flow regime is considered to be of the type generally referred to as annular mist. In this regime a gas core of large diameter with entrained droplets is flowing within a thin annular liquid film. This regime is not adequately represented by the pure annular flow regime assumed in the analysis.

The relatively good agreement between the steady state and transient results affirms that the inertial effects in the inlet and discharge lines to the pump during transient tests are greater than that in the pump impeller, but that neither effect is significant to the analysis of pump performance.

The steady state two-phase data for higher void fractions could only be meaningfully correlated by assuming separated flow and accounting for the relative velocity of the phases by definition of an effective density at the pump inlet. This quantity is expressed in terms of the average volumetric concentration of the separated flow assumed and the relative velocity (slip flow) between the phases. Since little is known about the response of a centrifugal pump to a two-phase mixture, the analysis performed on the data obtained from the steady state and transient tests on the Mod-1 pump has dictated two important conclusions: 
(1) To allow quantification of two-phase pump performance as a function of void fraction, special provisions must be made for ensuring that a homogeneous two-phase mixture enters the pump and several performance data points must be taken at a given void fraction. To avoid possible system effects, the homogeneous mixture should be obtained by a slow decompression process to achieve quasi-steady state conditions. An on-line computerized data acquisition system would significantly improve the quality of the data by allowing simultaneous acquisition of all measurable variables.

(2) With quantification of pump performance for homogeneous flow, the separate contribution of the pump to LOCA-related phenomena can be determined (in the case of large breaks) for the first portion of the decompression process when the pump performance is degrading under the influence of homogeneous two-phase tlow conditions. However, the contribution of the pump.throughout the complete blowdown process (particularly for small breaks) cannot be completely quantified (because of the eventual flow regime change in the system) until measurement techniques are improved to the extent that measurement of the velocity and concentration profiles in a nonhomogeneous two-phase mixture is possible. 


\section{REFERENCES}

1. R. O. Niemi and A. G. Steamer, Steam Carryunder Measurement by Means of Two-Phase Pump Measurements, GEAP-3562 (October 1960).

2. G. L. Sozzi and G. W. Burnette, Two-Phase Pump Performance During Steady State Operation and During a Simulated LOCA Blowdown, NEDE-13239 (November 1971).

3. D. J. Olson, Experiment Data Report for Single-and Two-Phase Steady State Tests of the 1-1/2-I,oop Mod-1 Semiscale System Pump, ANCR-1150 (May 1974).

4. R. S. Alder, E. M. Feldman, D. J. Olson, P. A. Pinson, Experiment Data Report for 1-1/2-Loop Semiscale System Isothermal Tests 1001, 1002, and 1003, ANCR-1148 (May 1974).

5. R. S. Alder, E. M. Feldman, P. A. Pinson, Experiment Data Report for 1-1/2-Loop Semiscale System Isothermal Tests 1004 and 1006, ANCR-1 144 (March 1974).

6. R. S. Alder, E. M. Feldman, P. A. Pinson, Experiment Data Report for 1-1/2-Loop Semiscale System Isothermal Tests 1008 and 1010, ANCR-1 145 (March 1974).

7. E. M. Feldman and P. A. Pinson, Experiment Data Report for 1-1/2-Loop Semiscale System Isothermal Test 1011, ANCR-1 146 (March 1974).

8. E. M. Feldman, Experiment Data Report for 1-1/2-Loop Semiscale System Isothermal Test 1009, ANCR-1 152 (June 1974).

9. E. M. Feldman, Experiment Data Report for 1-1/2-Loop Semiscale System Isothermal Test 1014, ANCR-1 147 (March 1974).

10. D. J. Olson, "Modeling Philosophy and Selection of Pumps for Use in Small Blowdown Experiments", Topical Meeting on Water Reactor Safety, CONF-730-304, pp 758-766 (March 26-28, 1973).

11. S. A. Naff and P. A. Pinson, 1-1/2-Loop Semiscale Isothermal Test Program -- Program and System Description in Support of Experiment Data Reports, ANCR-1143 (February 1974).

12. A. J. Stepanoff, Centrifugal and Axial Flow Pumps, John Wiley and Sons, Inc., Second Edition (1957).

13. V. L. Streeter and E. B. Wylie, Hydraulic Transients, McGraw-Hill Book Company (1967). 
14. G. W. Govier and K. Aziz, The Flow of Complex Mixtures in Pipes; Wan Nostrandw Reinhold Company (1972).

15. G. A. Hughmark and B. S. Pressberg, "Holdup and Pressure Drop with Gas-Liquid Flow in a Vertical Pipe", AIChE Journal, 7, 677 (1961).

16. N. Zuber and J. Findlay, "Average Volumetric Concentration in. Two Phase Flow Systems", Trans. ASME J. Heat Transfer, Sec. C, Vol. 87, p 453. 


\section{THIS PAGE \\ WAS INTENTIONALLY \\ LEFT BLANK}


APPEN.DIX A

\section{:PRESENTATION OF TRANSIENT DATA FROM}

SEMISCALE ISOTHERMAL:BLOWDOWN TESTS 
THIS PAGE

\section{WAS INTENTIONALLY \\ LEFT BLANK}




\section{APPENDIX A \\ PRESENTATION OF TRANSIENT DATA FROM \\ SEMISCALE ISOTHERMAL BLOWDOWN TESTS}

Figures A-1 through A-27 of this appendix provide the isothermal blowdown test data relative to pump behavior which were used in the analysis presented in this report. Included are plots of pump differential pressure, pump speed, pump torque, and pump inlet and outlet densities, void fraction, and volumetric flow rate. The data presented are from Tests 1008,1009 , and 1010. The data from all tests in the isothermal series, with the exception of void fraction and volumetric flow rate, are generally available in the data reports issued for the isothermal test series $[\mathrm{A}-1-\mathrm{A}-6]$. The void fraction and volumetric flow rate data required special data processing and were prepared in conjunction with the pump analysis. Void fraction results were calculated by combining the measured density (assuming homogeneous flow) and the density of each phase in the mixture (derived from saturation properties through use of measured fluid temperature) as follows:

where

$$
\rho_{\mathrm{m}}=\alpha \rho_{\mathrm{g}}+(1-\alpha) \rho_{f}
$$

$$
\begin{array}{ll}
\rho_{\mathrm{m}} & =\text { measured density } \\
\rho_{\mathrm{g}} & =\text { saturated vapor density } \\
\rho_{\mathrm{f}} & =\text { saturated liquid density } \\
\alpha & =\text { void fraction. }
\end{array}
$$

Volumetric flow rates were obtained by combining the momentum flux $\left(\rho \mathrm{V}^{2}\right)$ measurement obtained from the drag discs with the measured density. 


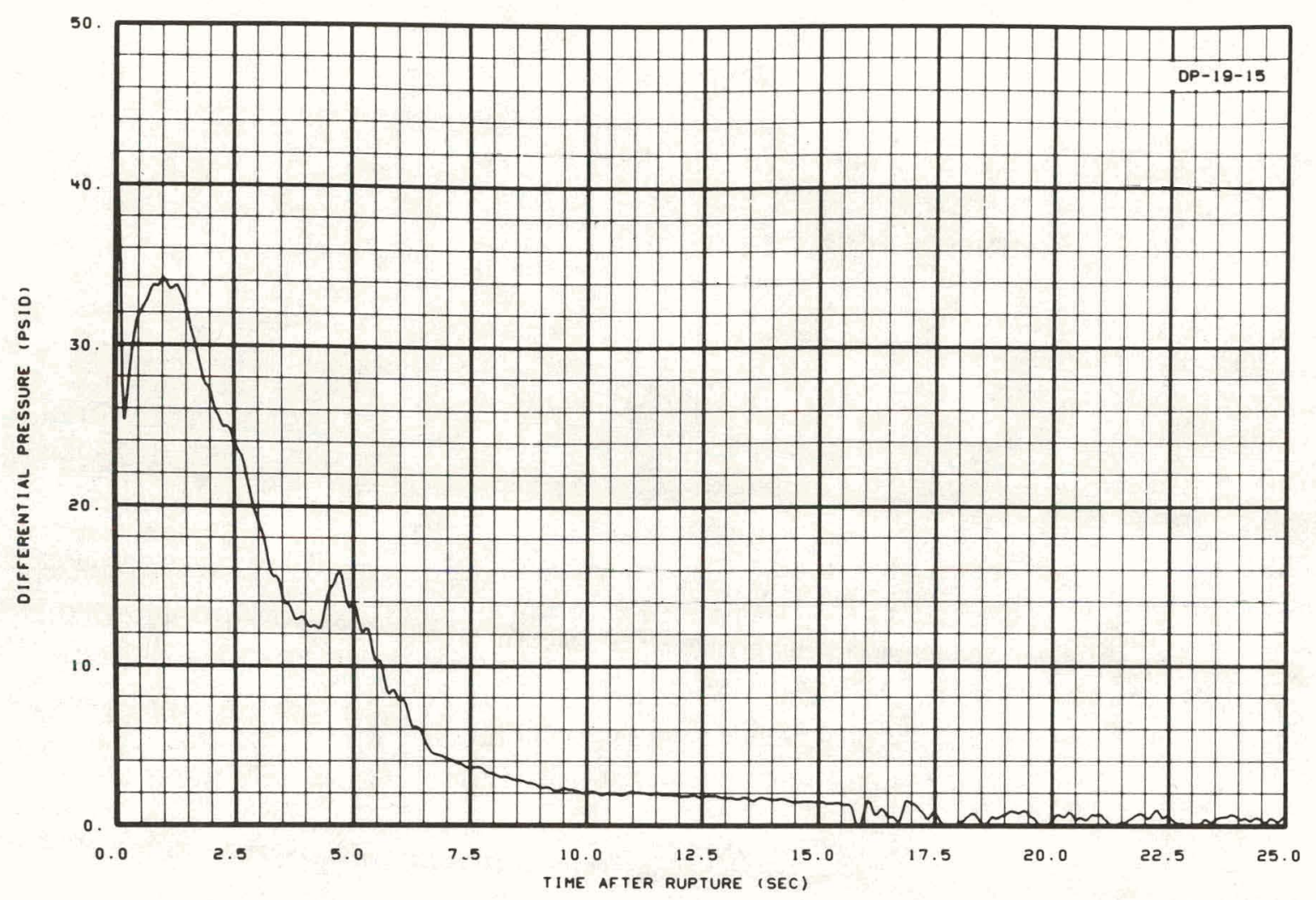

Fig. A-1 Pump differential pressure -- Test 1008.

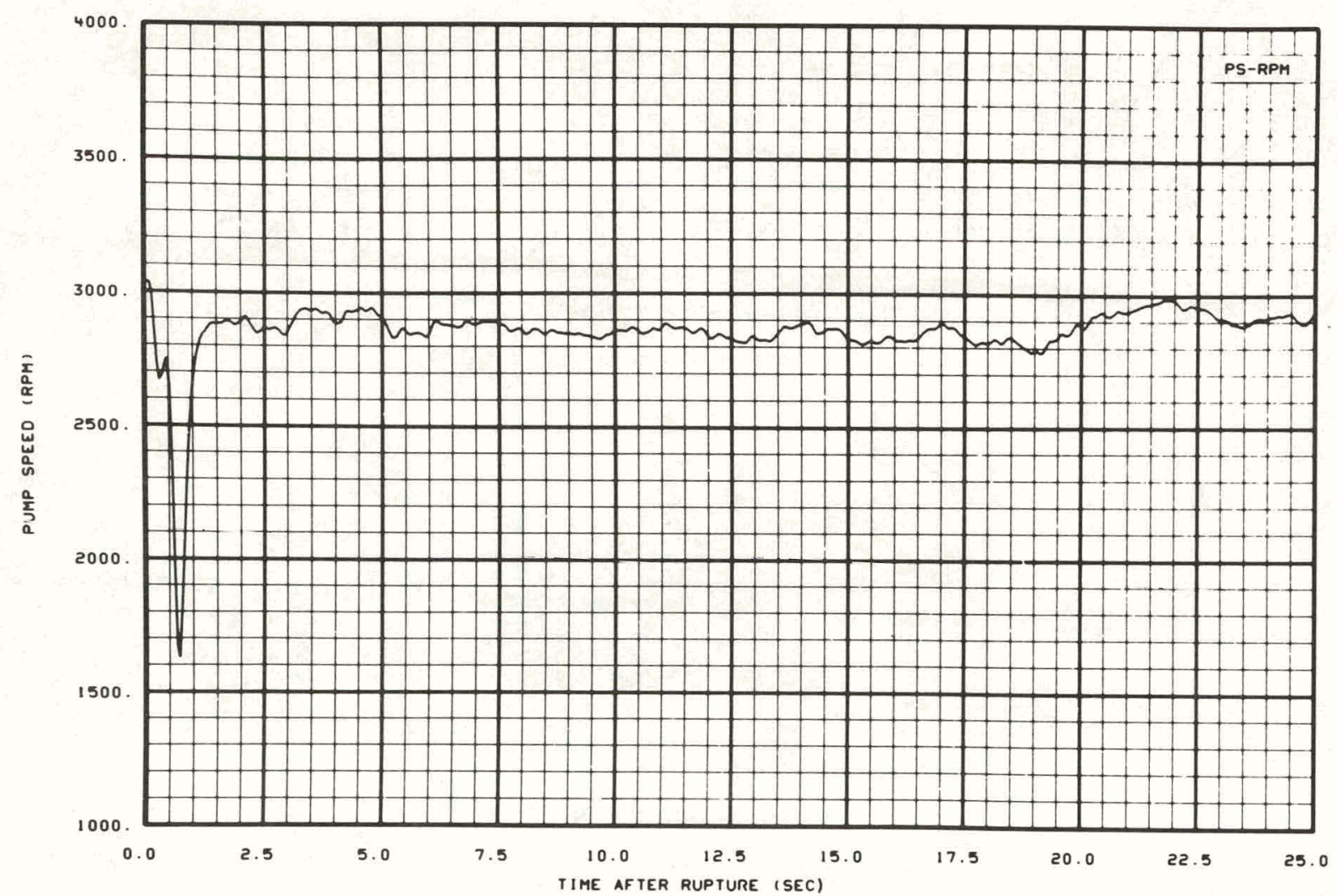

Fig. A-2 Pump speed -- Test 1008. 


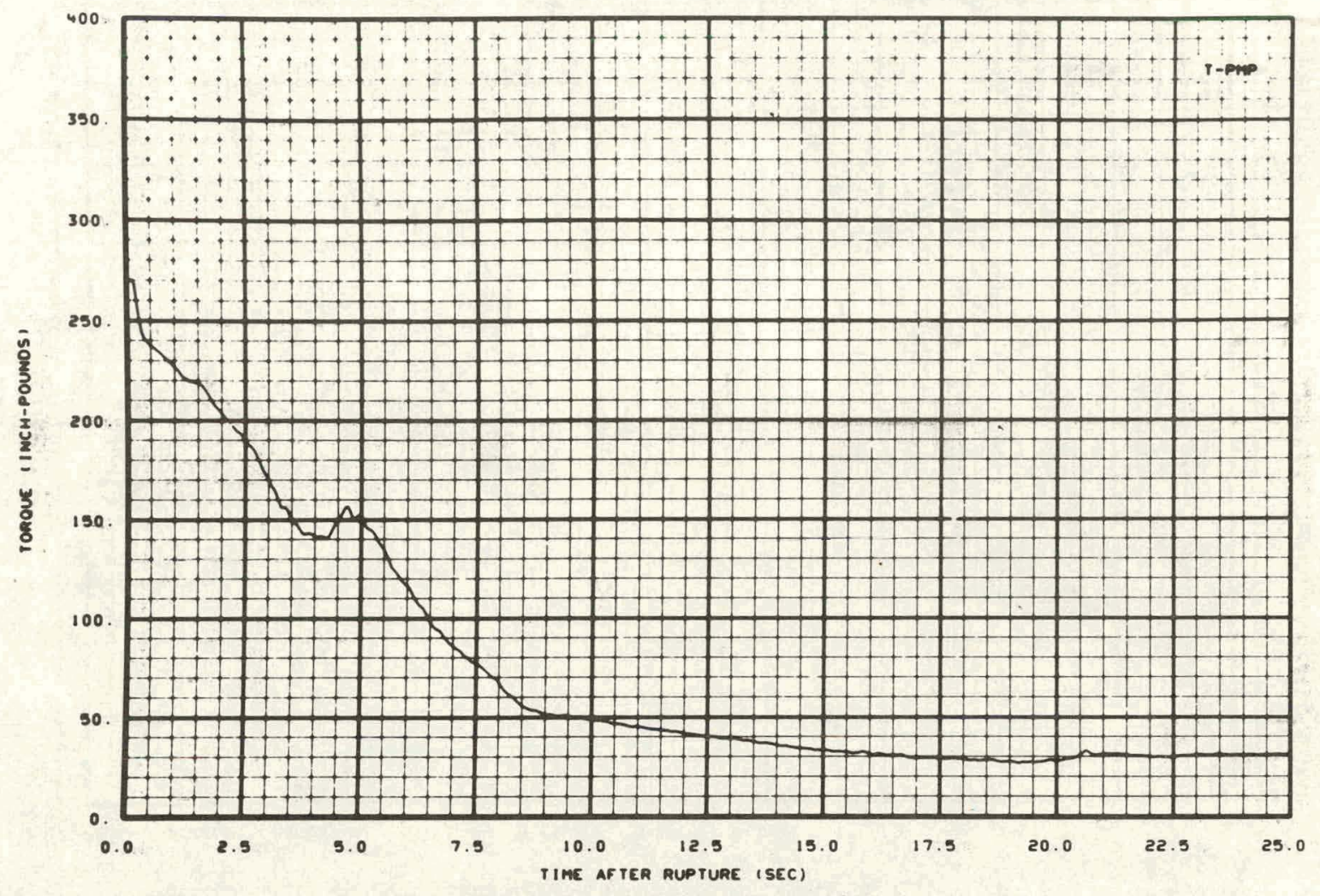

Fig. A-3 Pump torque -- Test 1008.

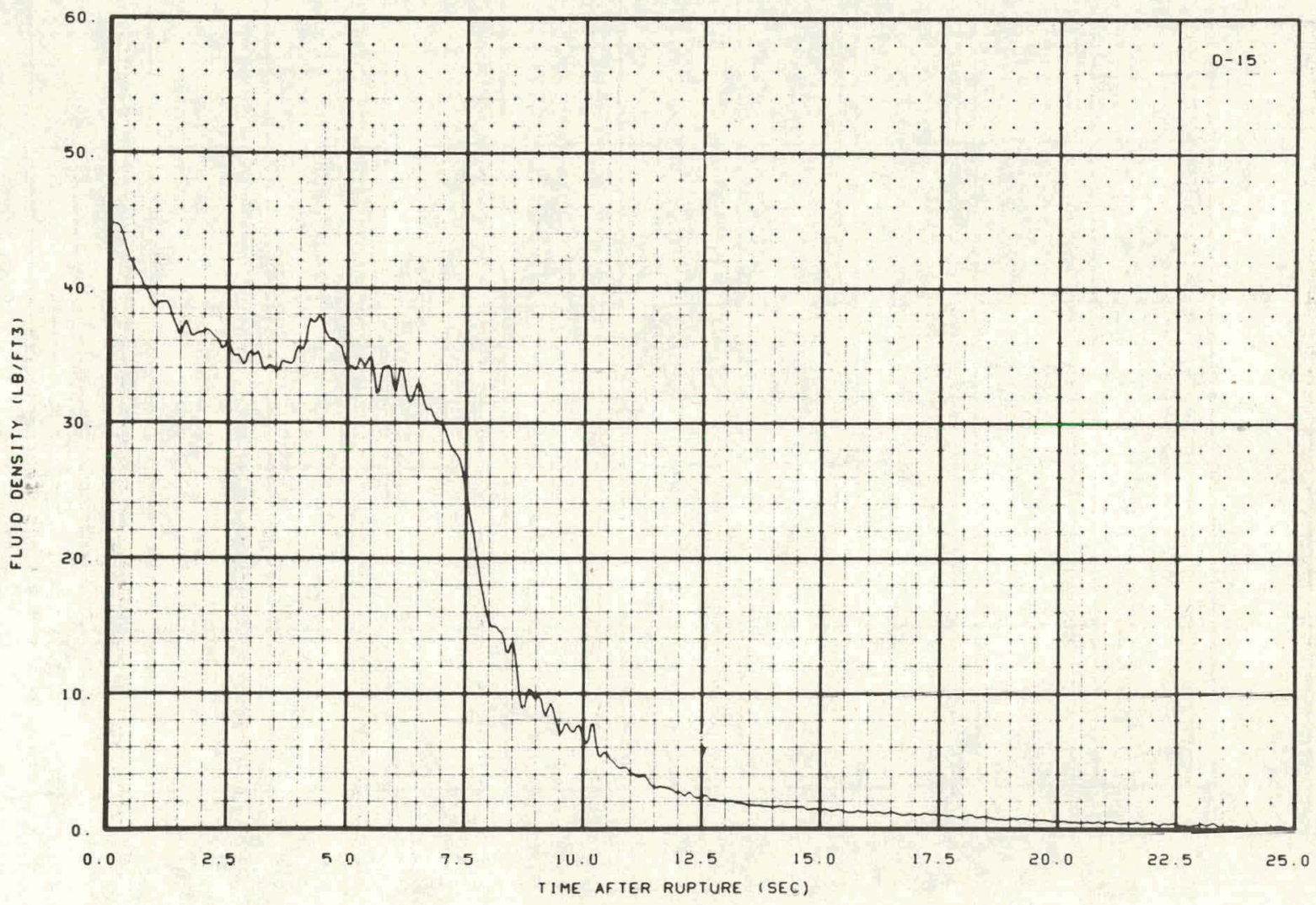

Fig. A-4 Pump inlet fluid density -- Test 1008. 


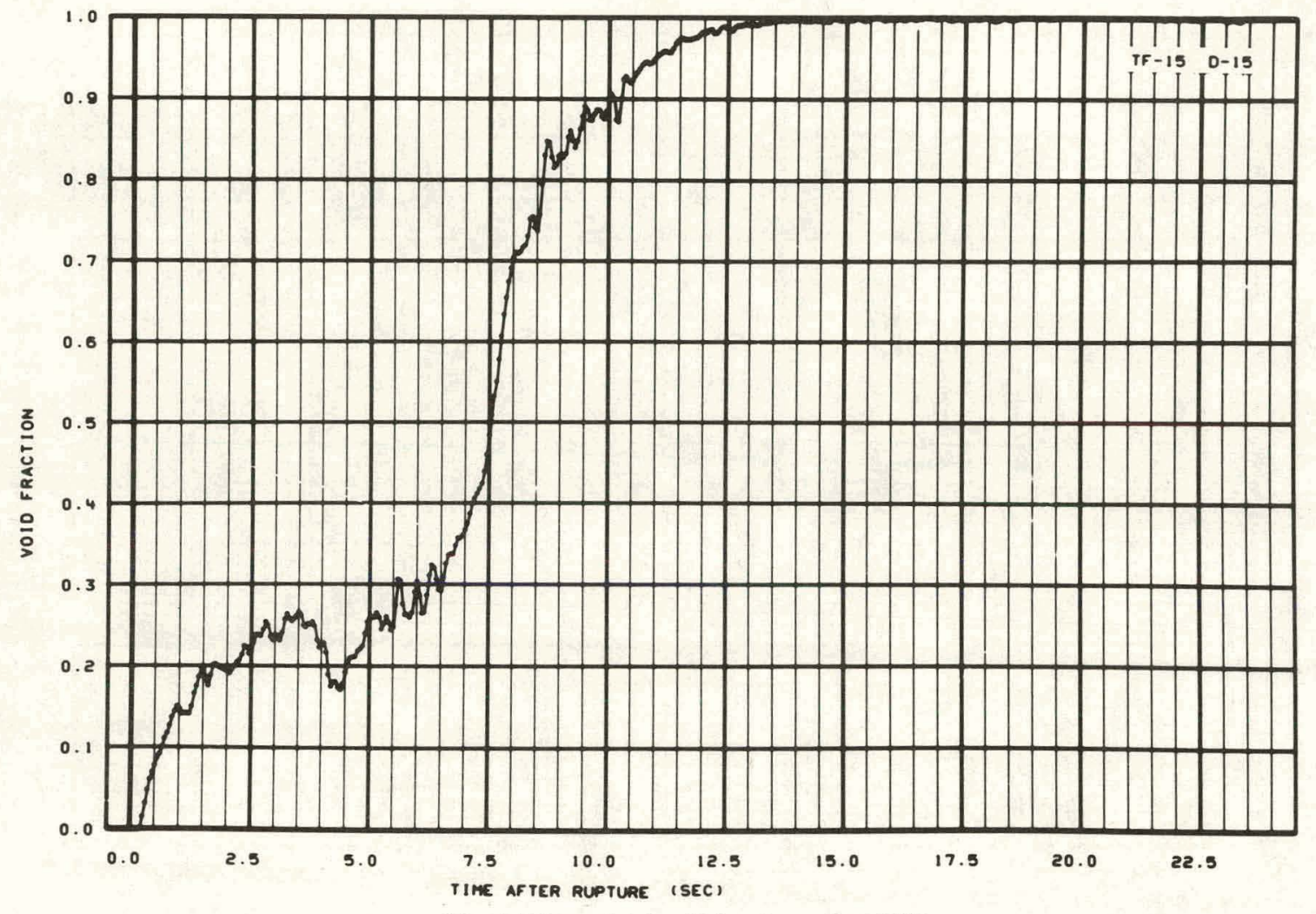

Fig. A-5 Pump inlet void fraction -- Test 1008.

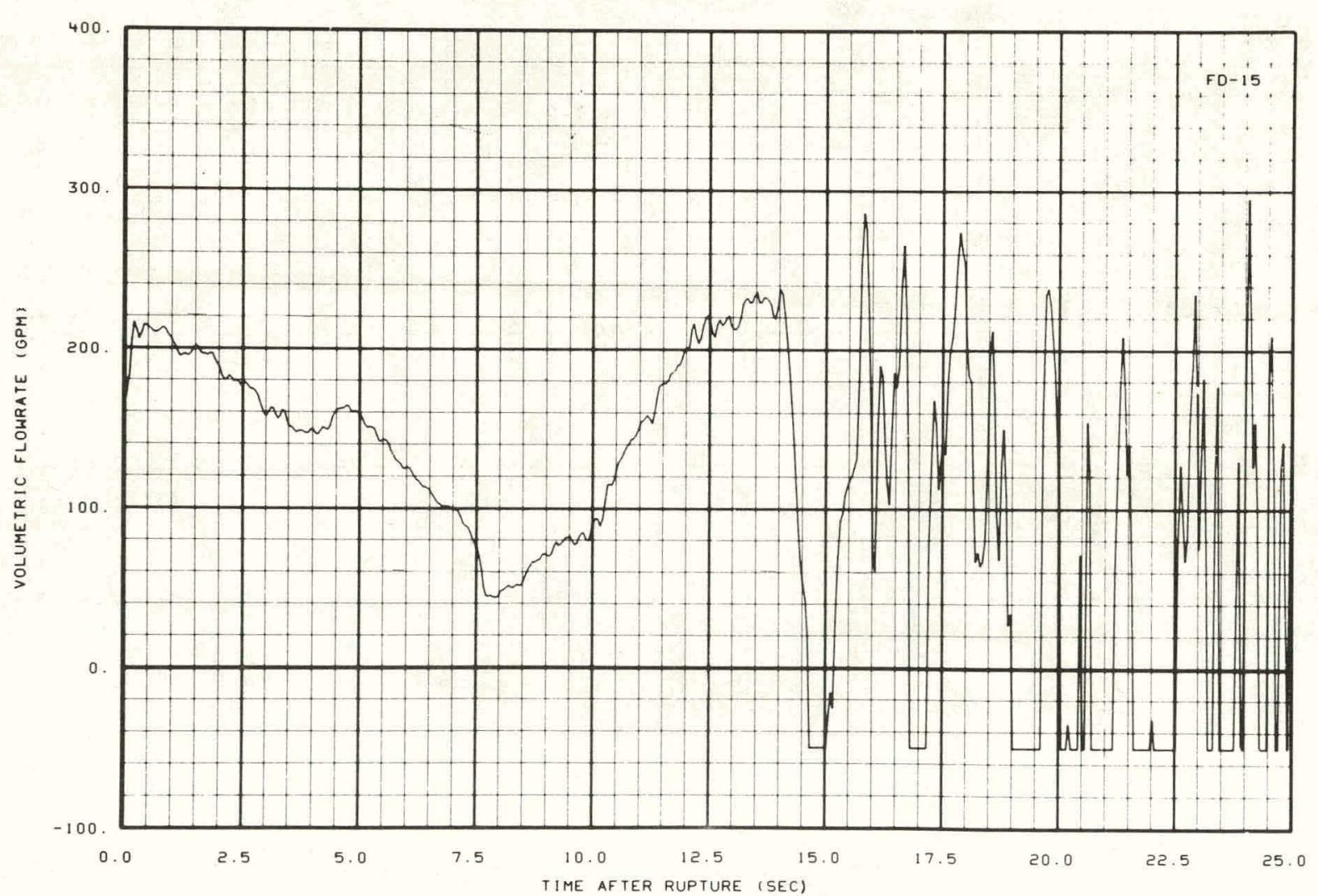

Fig. A-6 Pump iniet volumetric flow rate -- Test 1008. 


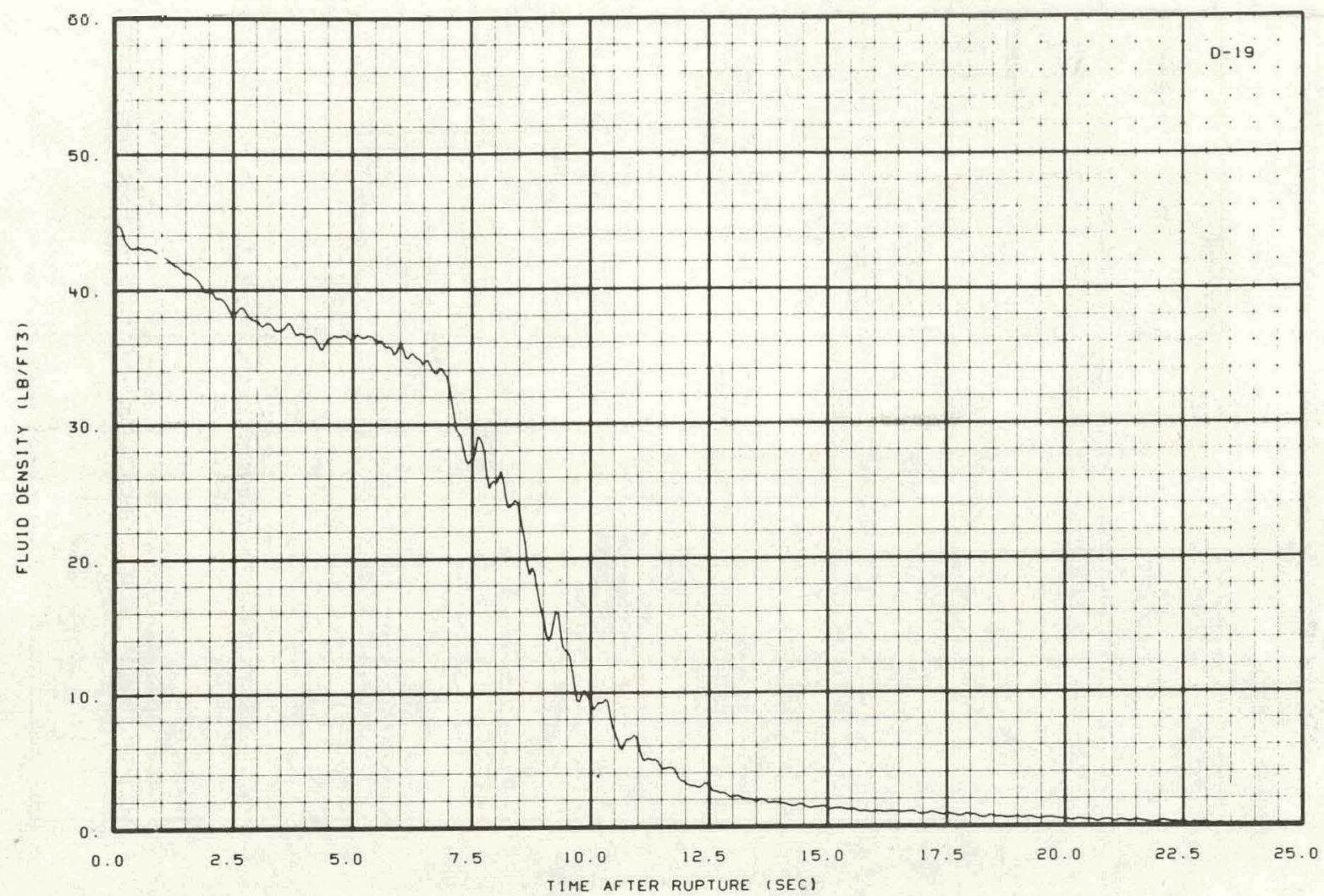

Fig. A-7 Pump outlet fluid density -- Test 1008.

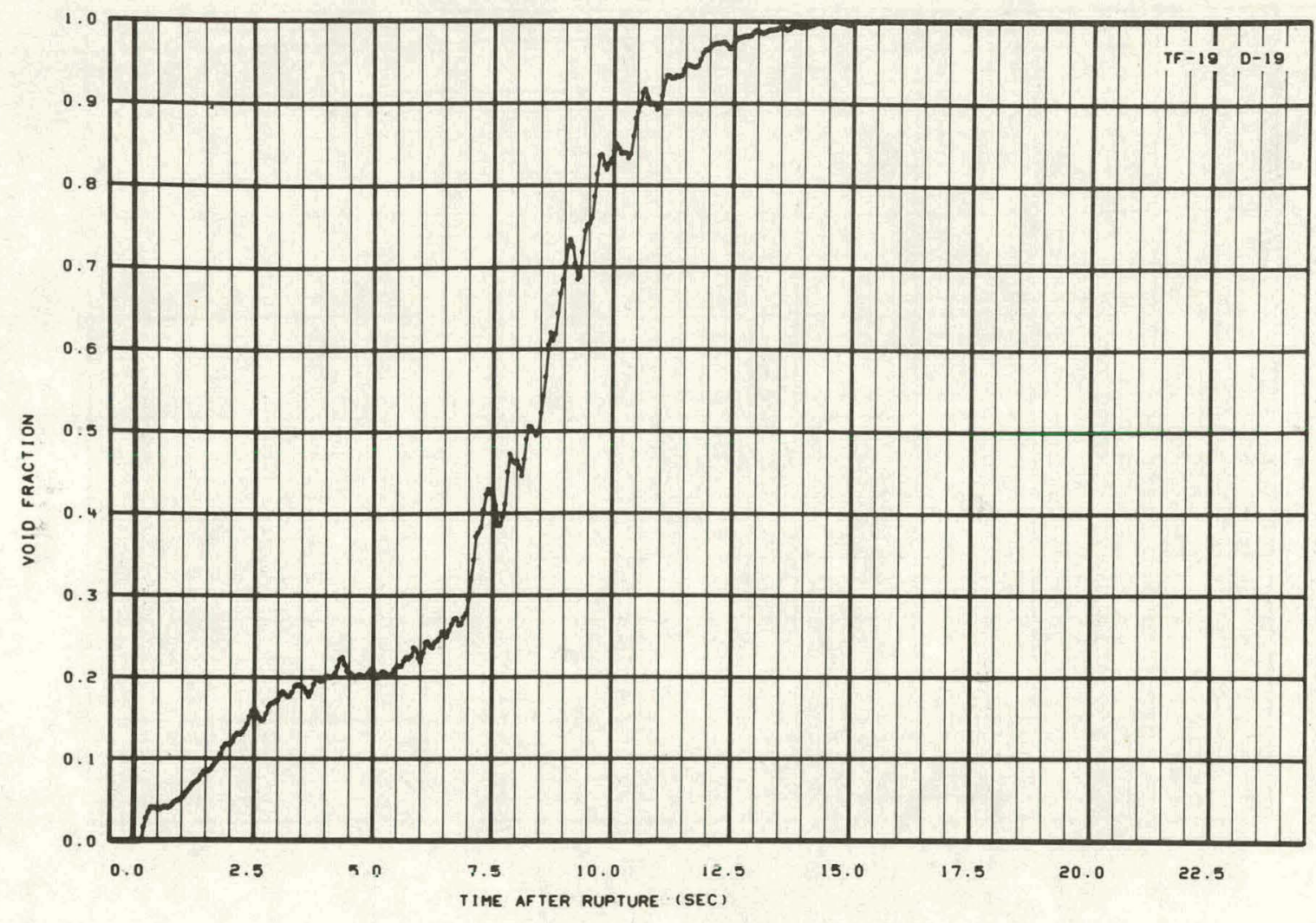

Fig. A-8 Pump outlet void fraction -- Test 1008. 


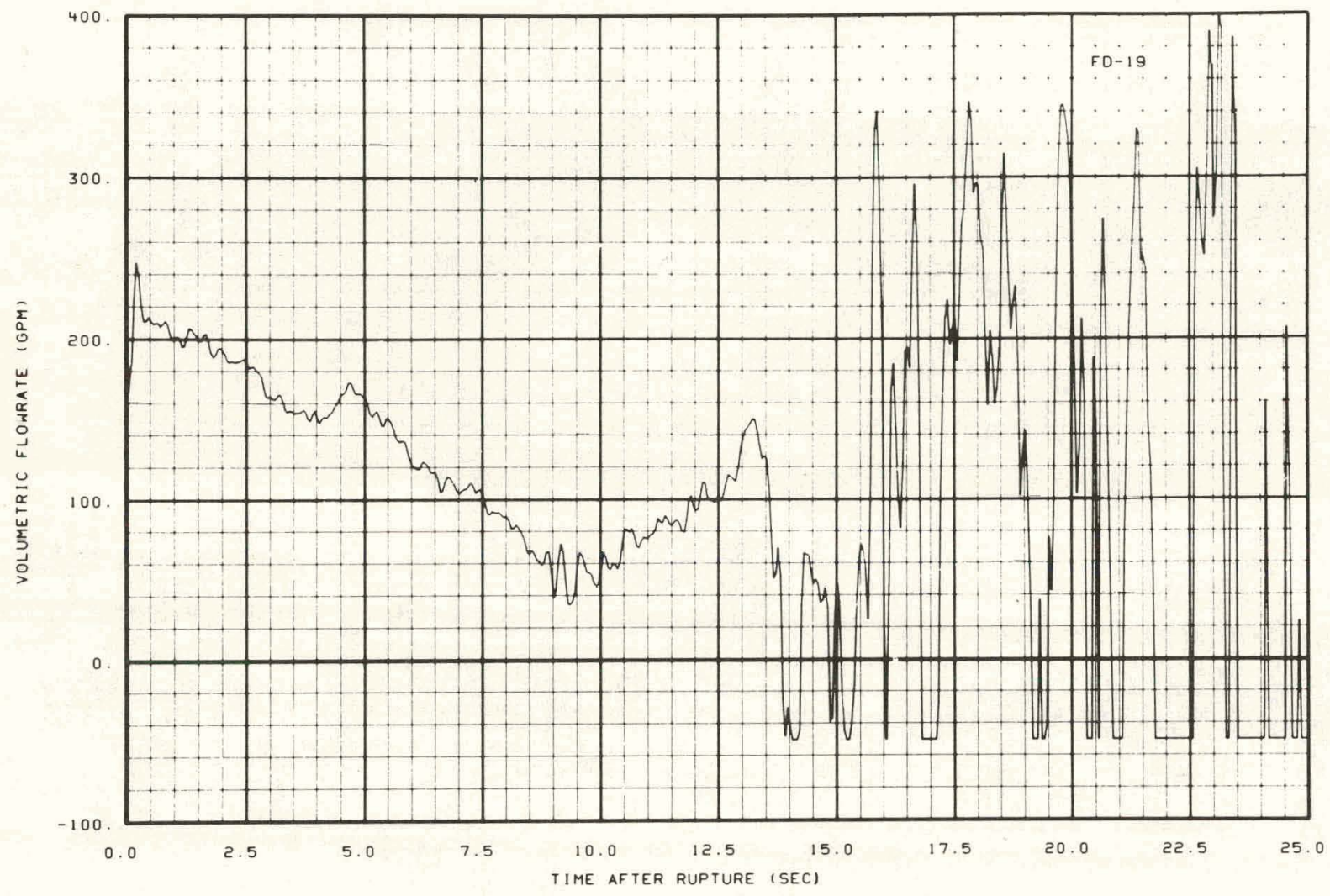

Fig. A-9 Pump outlet volumetric flow rate -- Test 1008 .

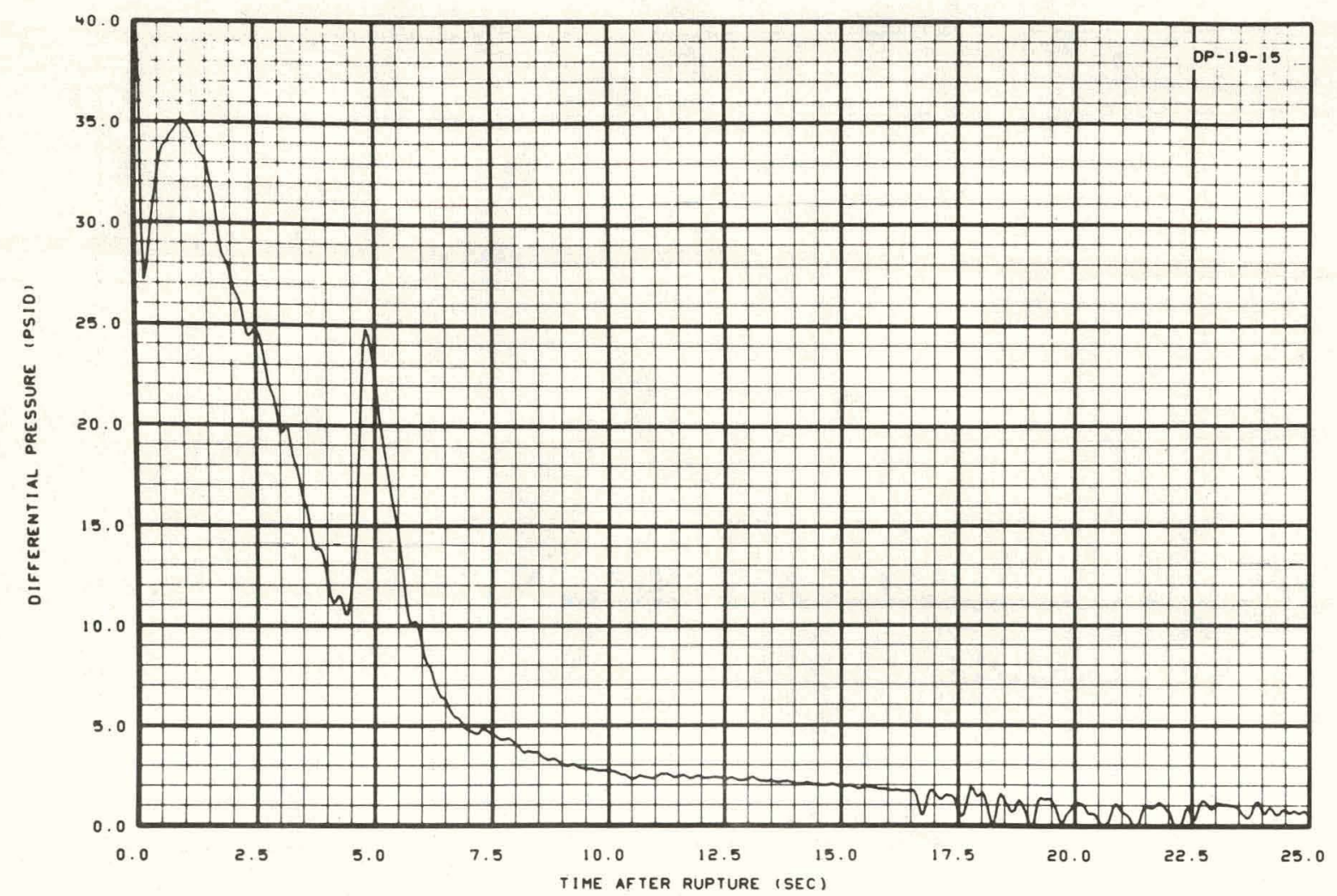

Fig. A-10 Pump differential pressure -- Test 1009 


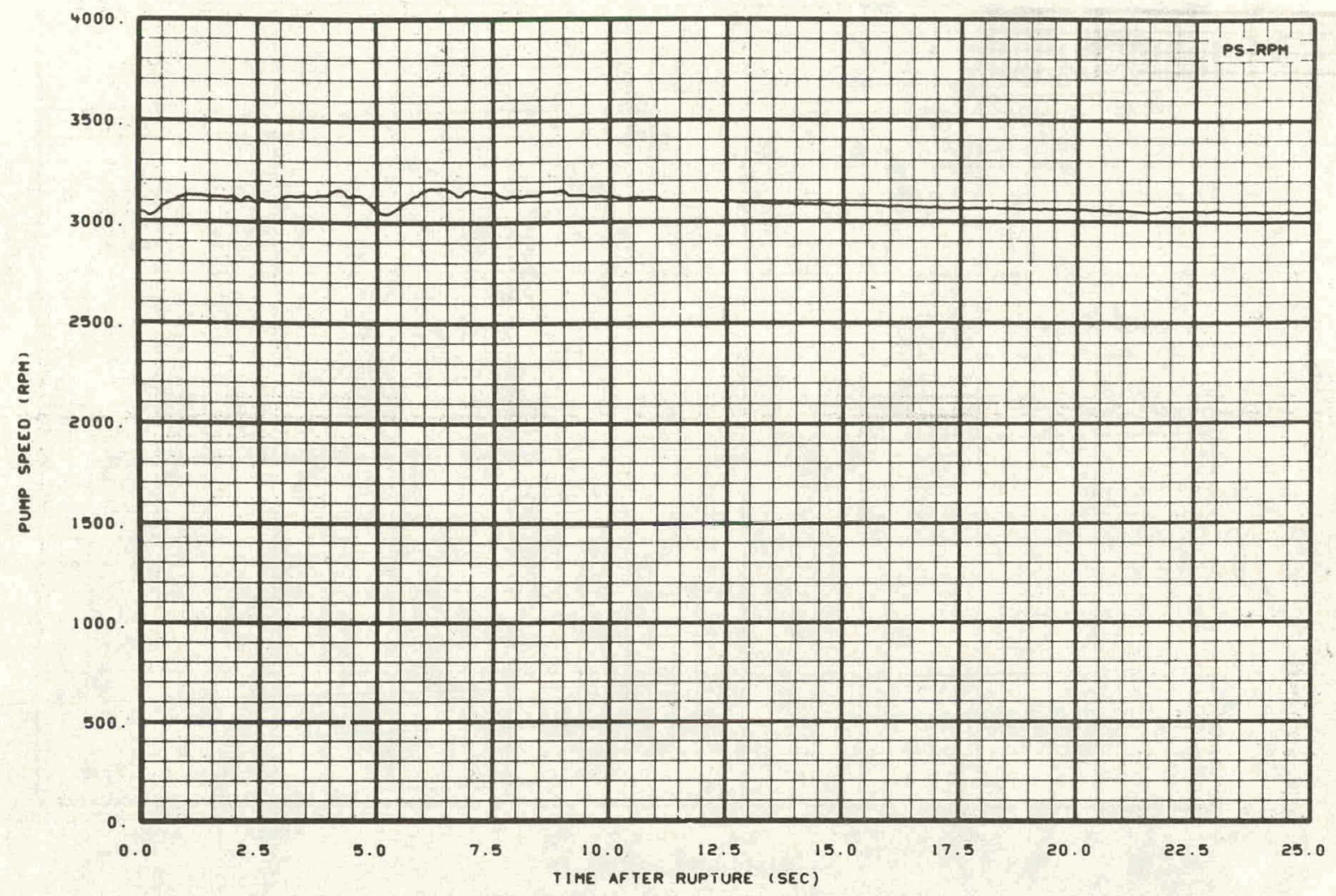

Fig. A-11 Pump speed -- Test 1009.

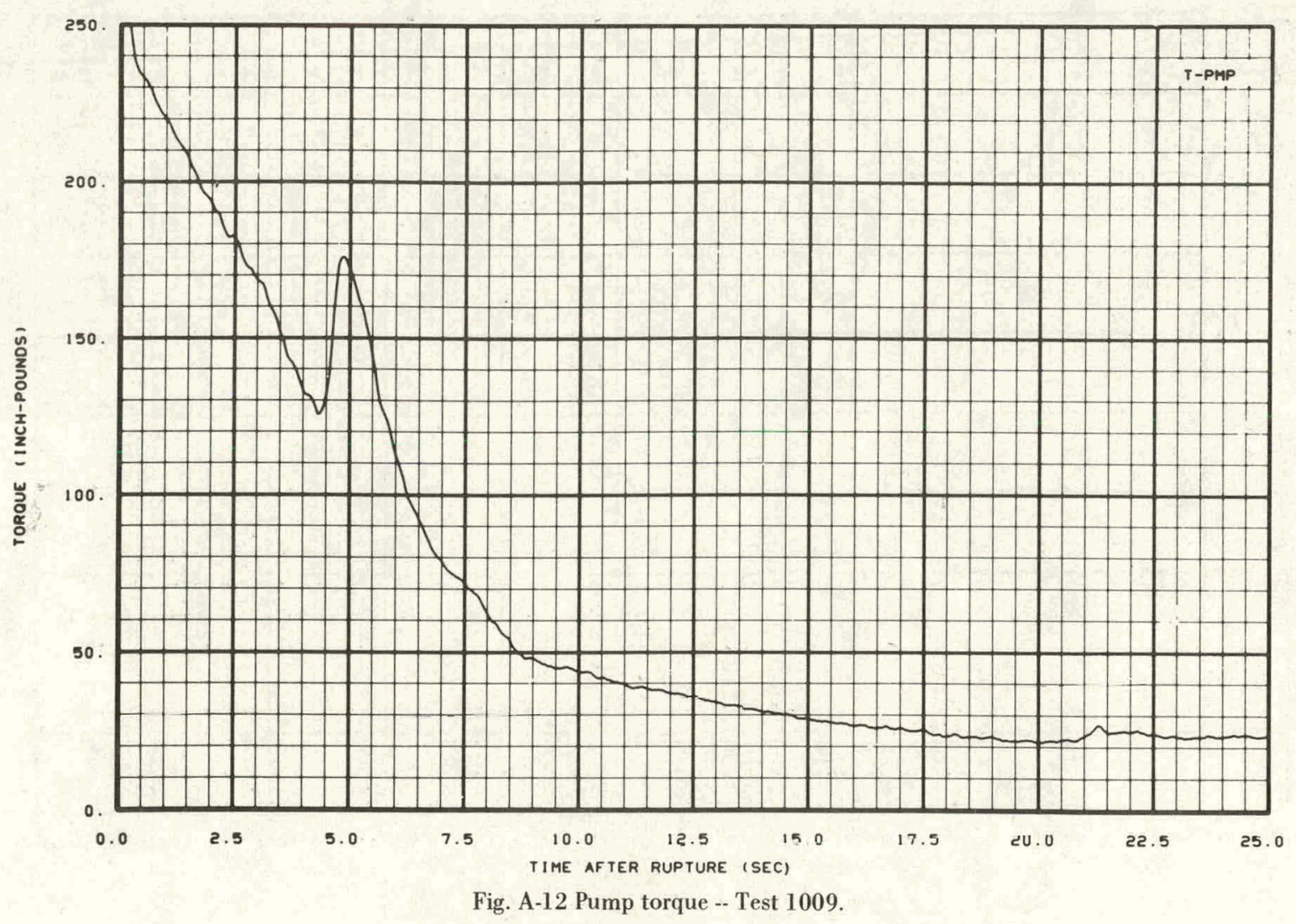




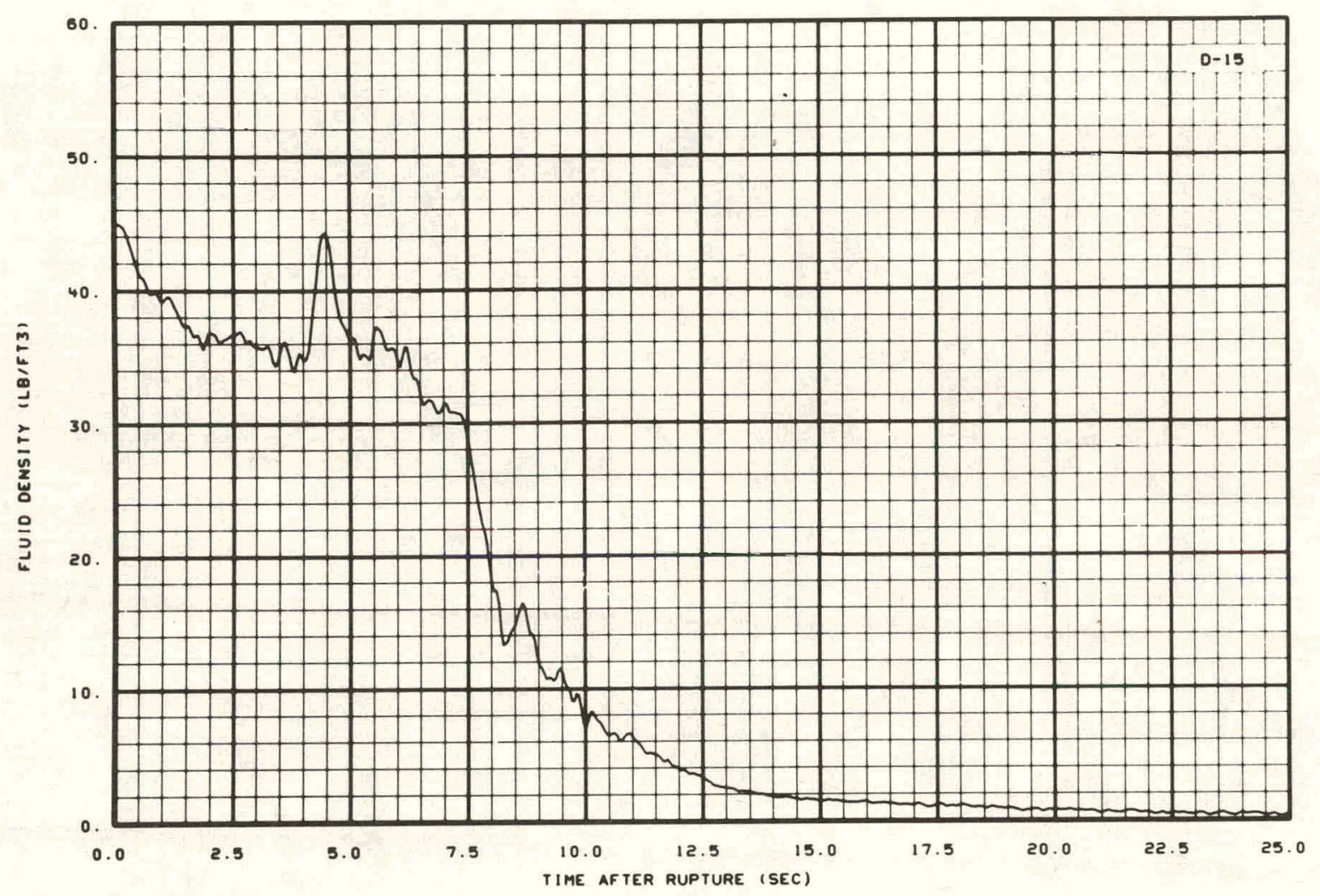

Fig. A-13 Pump inlet fluid density -- Test 1009.

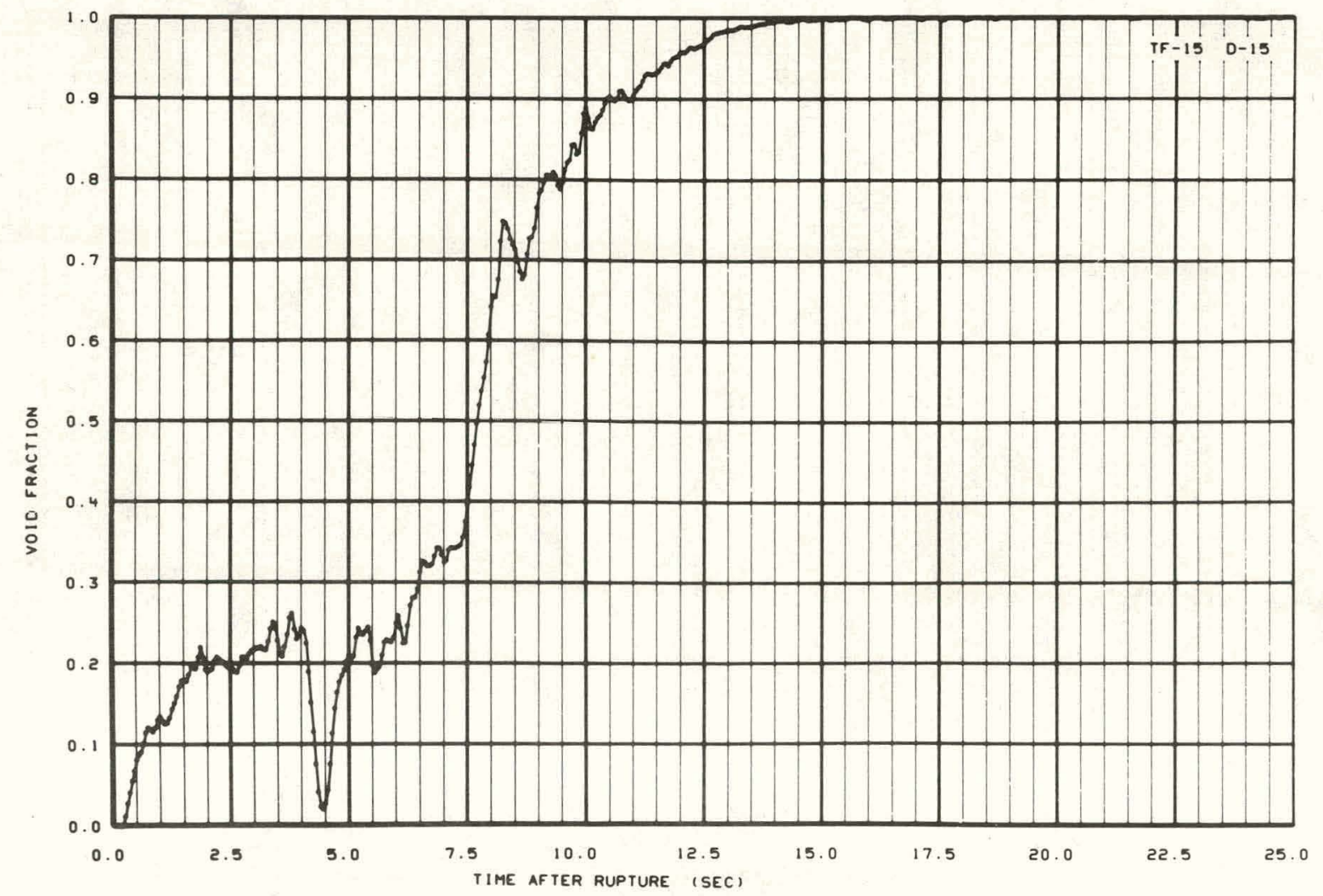

Fig. A-14, Pump inlet void fraction -- Test 1009. 


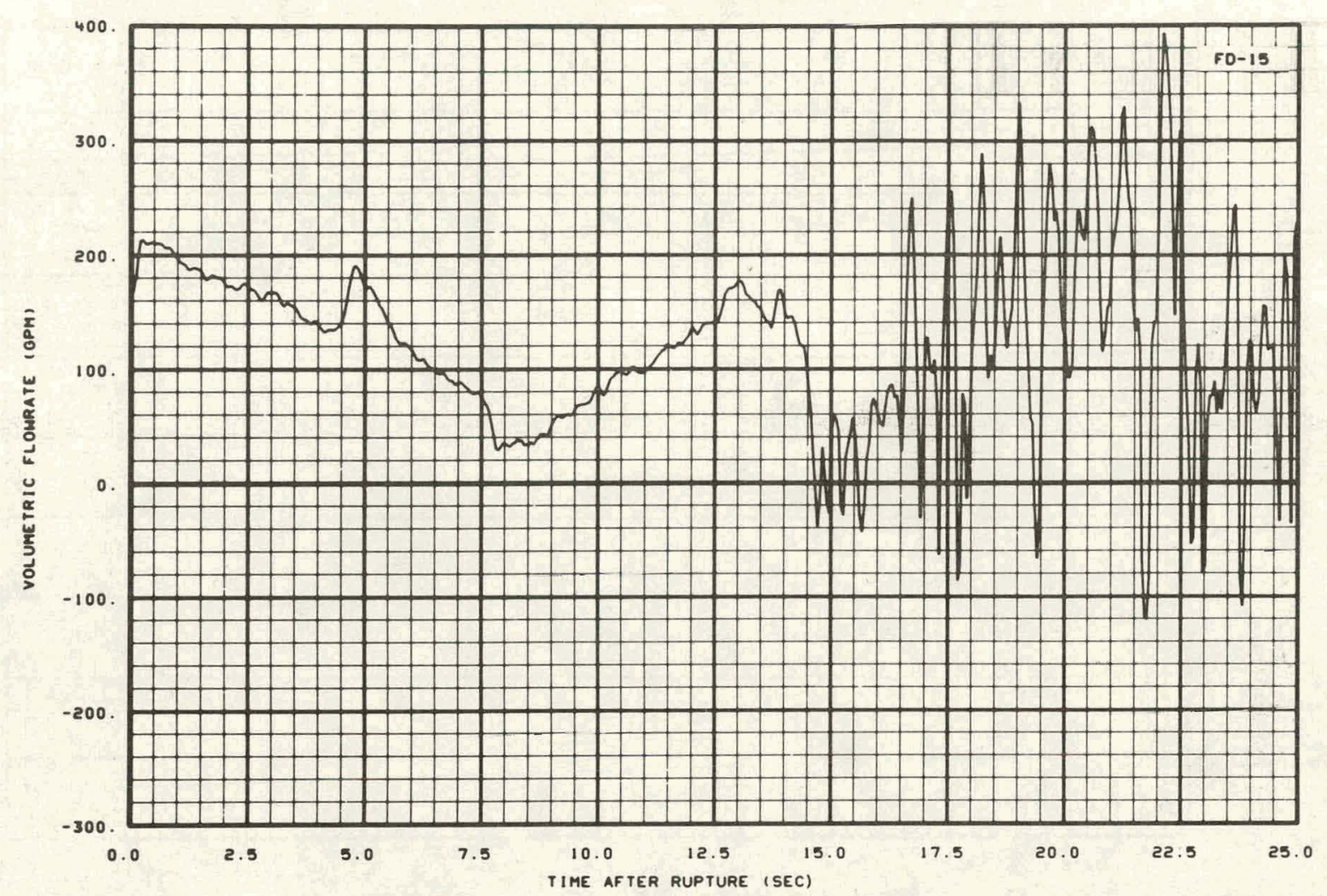

Fig. A-15 Pump inlet volumetric flow rate -- Test 1009.

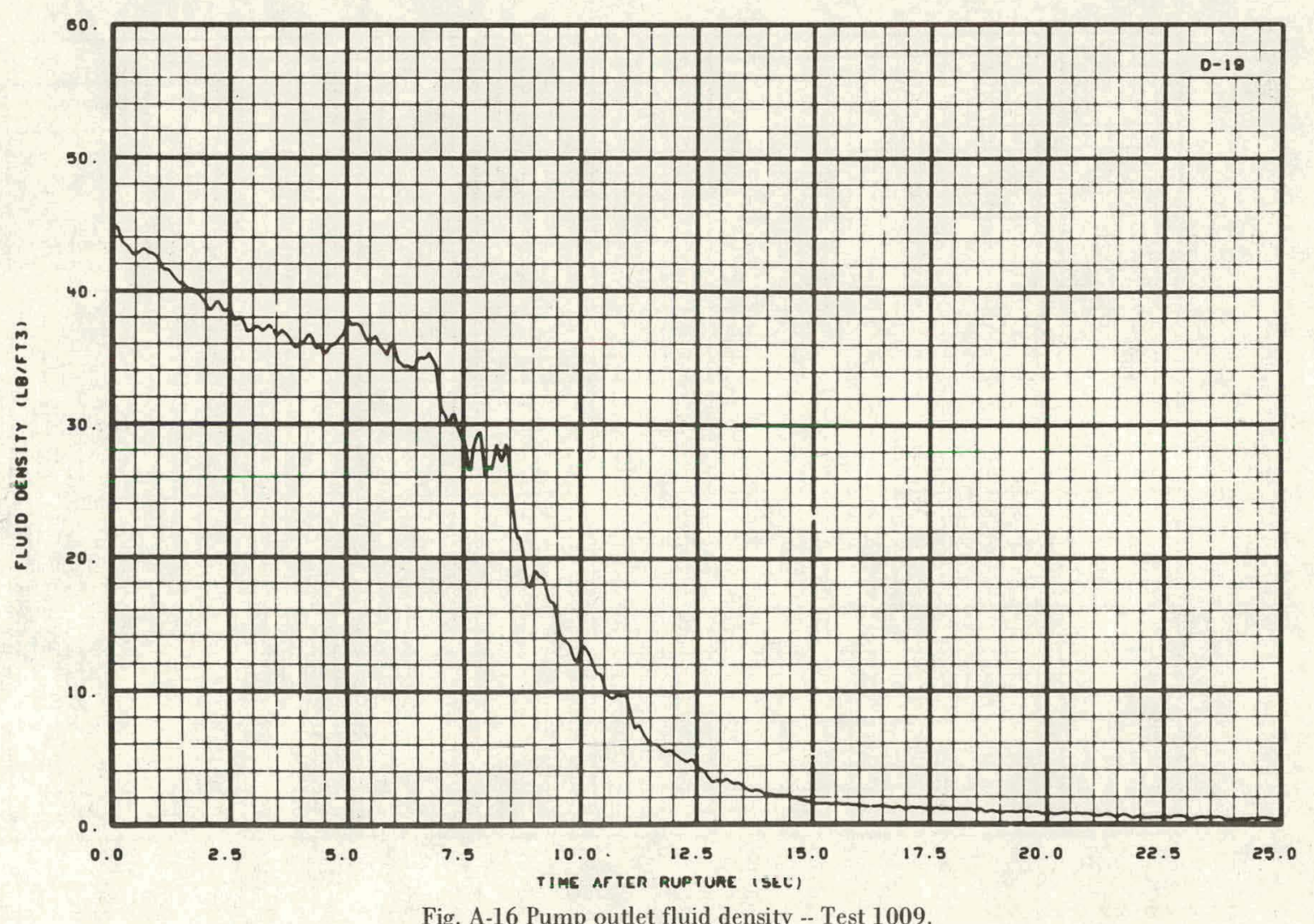




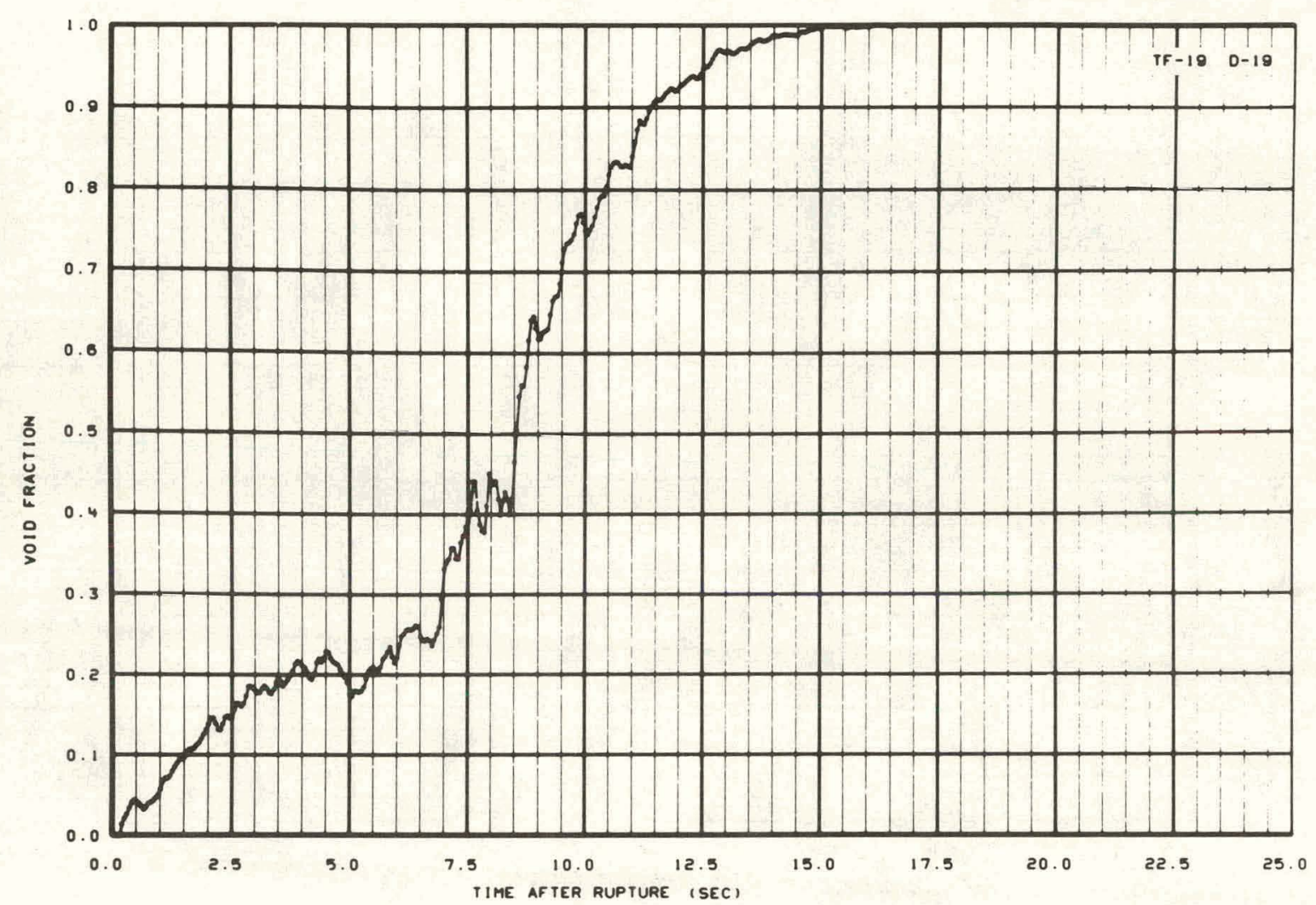

Fig. A-17 Pump outlet void fraction -.- Test 1009.

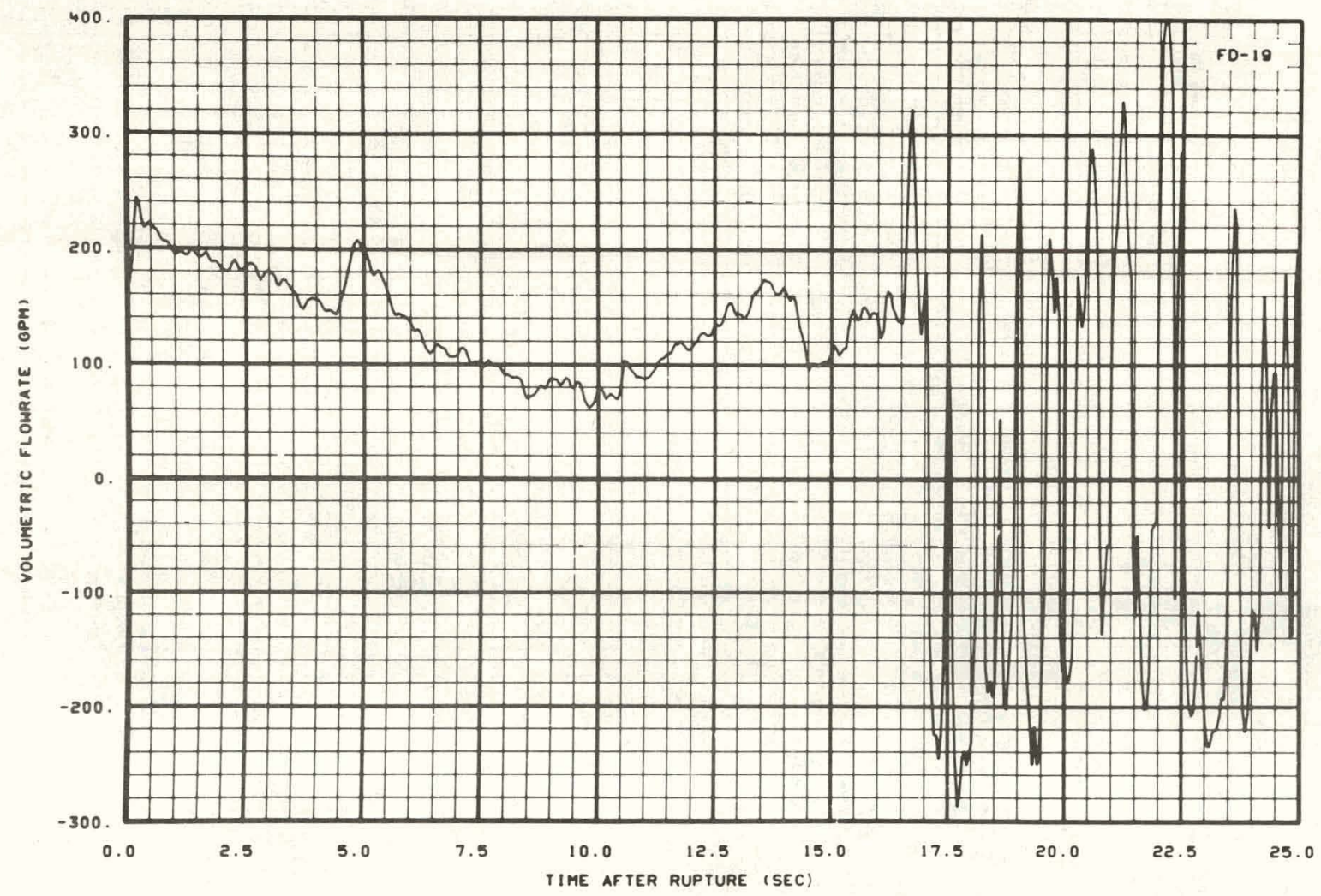

Fig. A-18 Pump outlet volume tric flow rate -- Test 1009. 


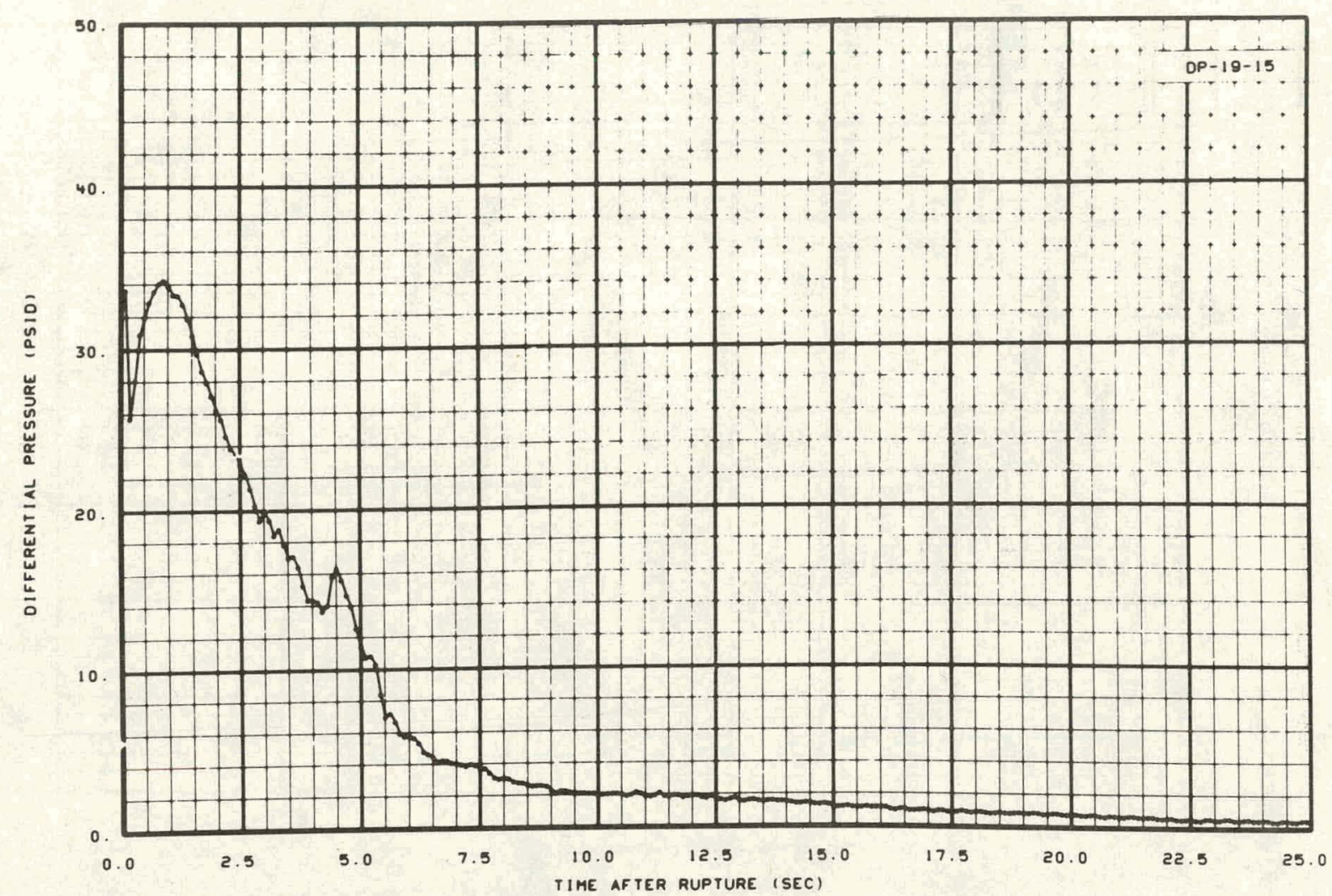

Fig. A-19 Pump differential pressure -- Test 1010.

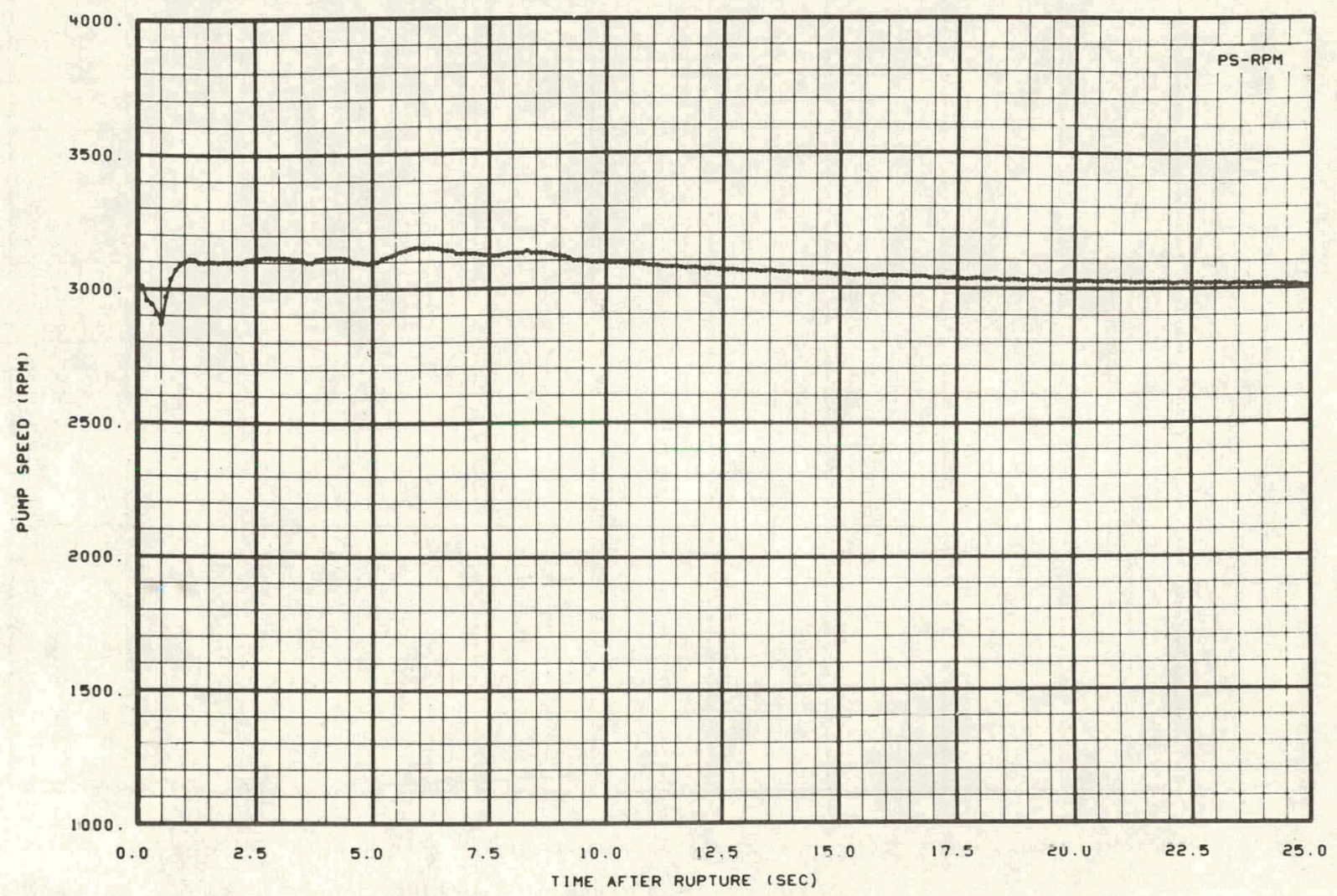

Fig. A-20 Pump speed -- Test 1010. 


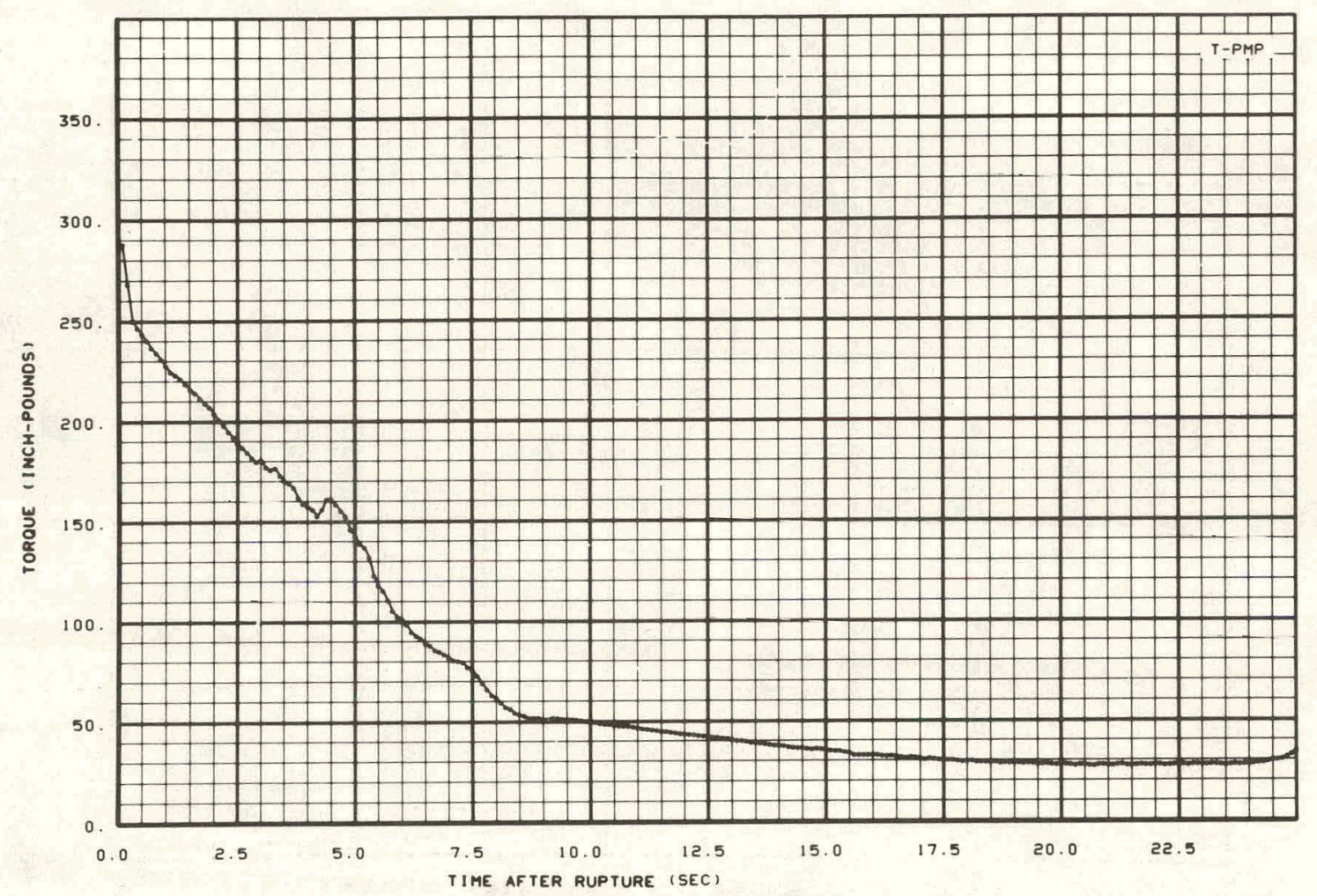

Fig. A-21 Pump torque -- Test 1010.

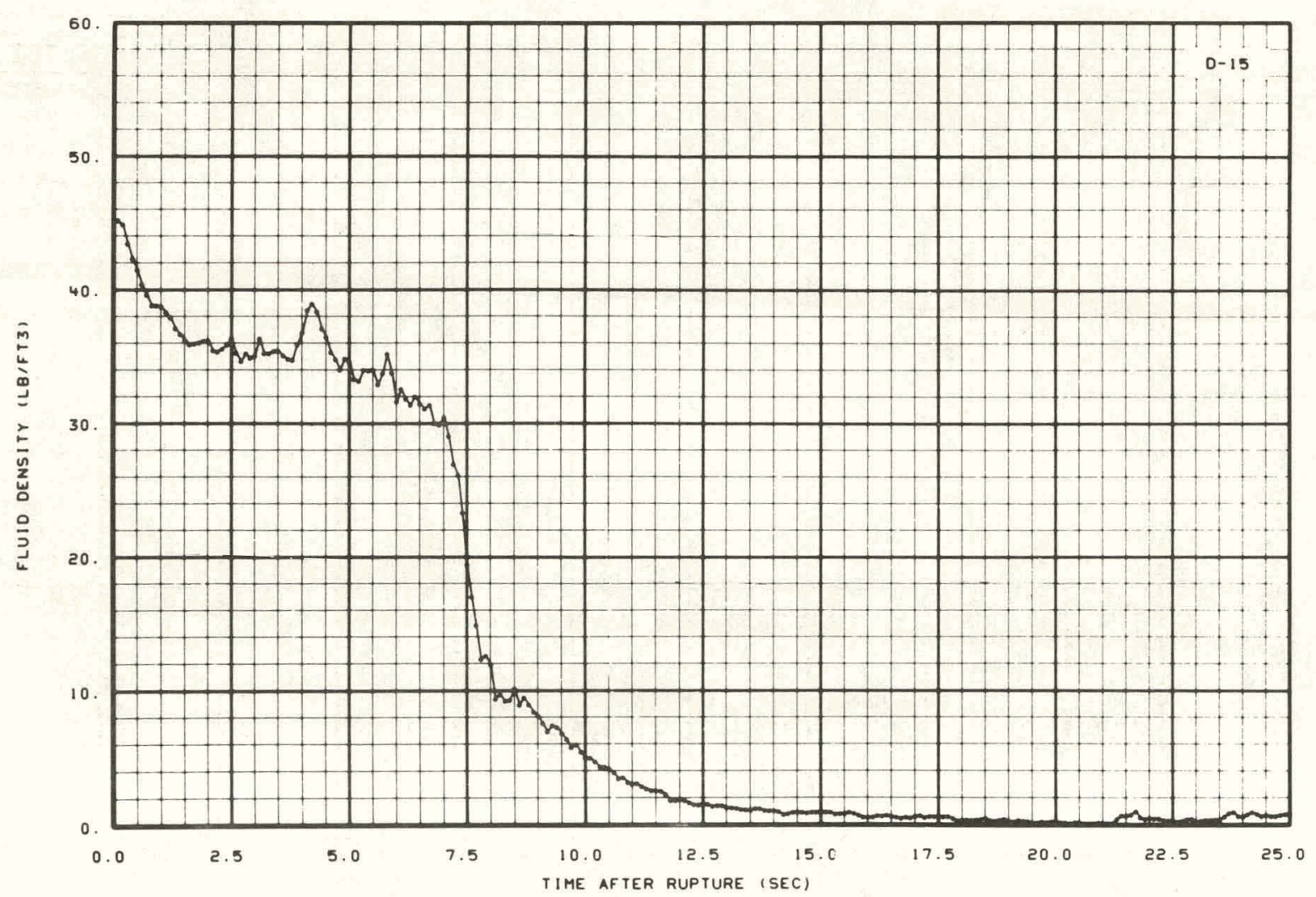

Fig. A-22 Pump inlet fluid density -- Test 1010. 


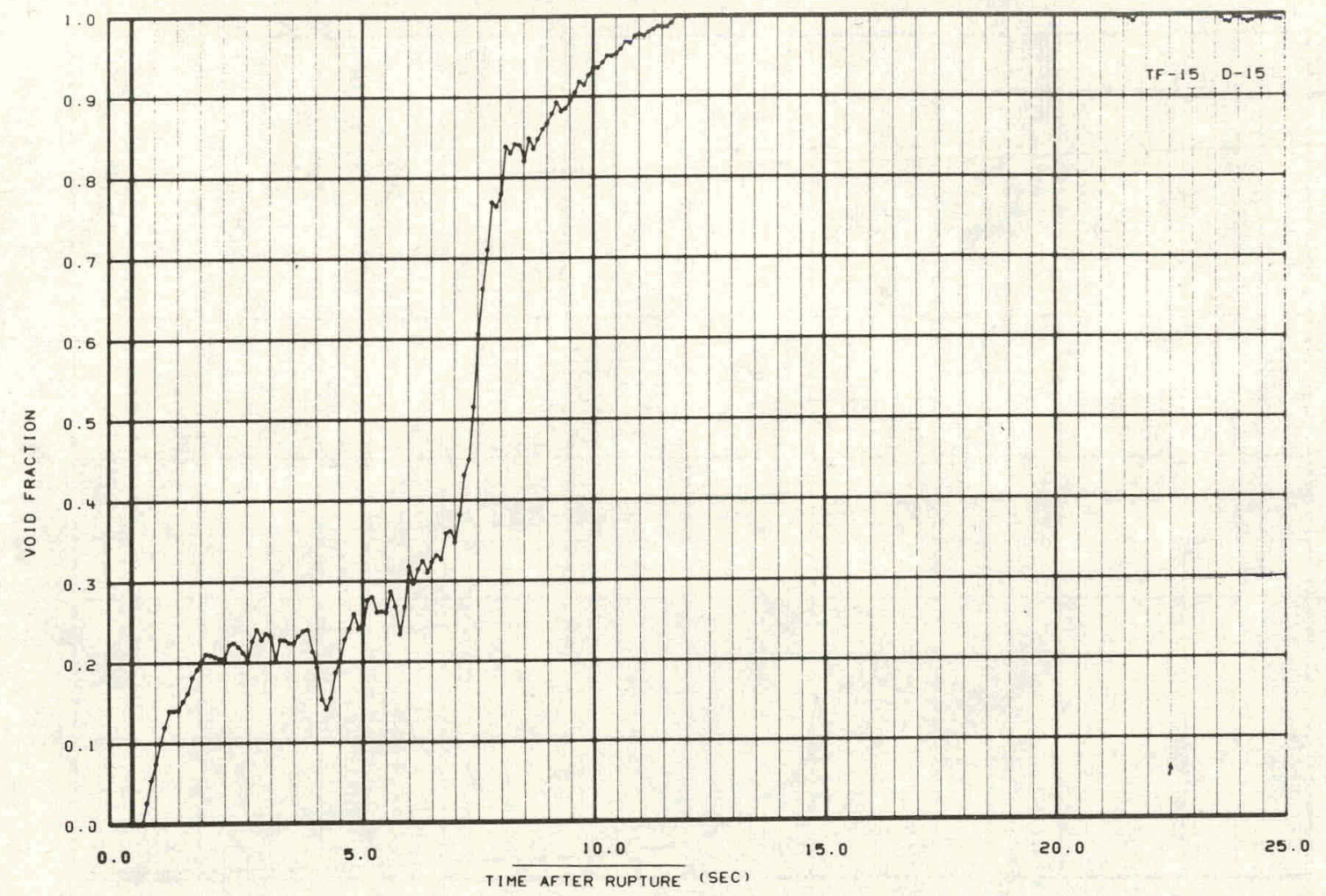

Fig. A-23 Pump inlet void fraction -- Test 1010.

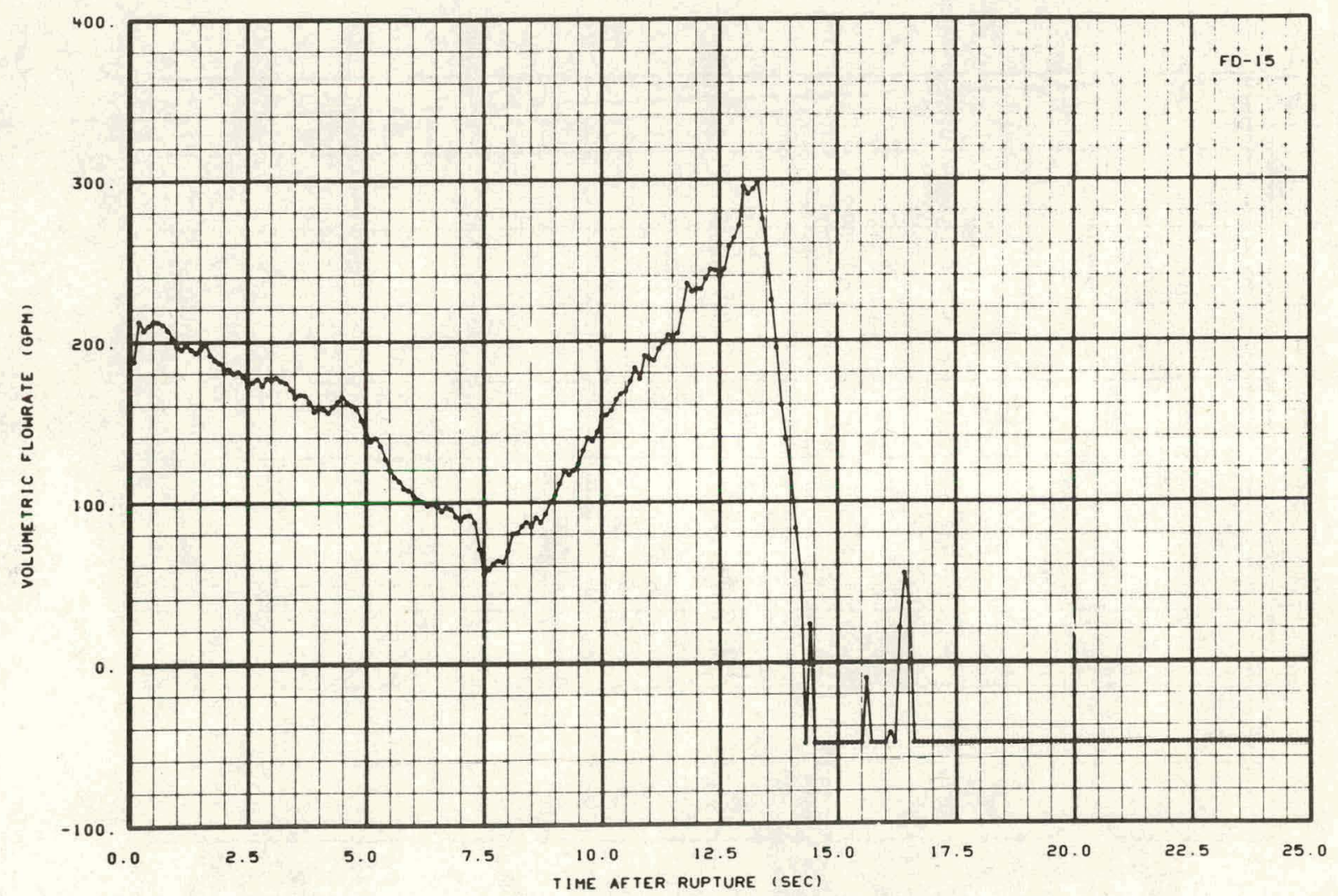

Fig. A-24 Pump inlet volumetric flow rate -- Test 1010. 


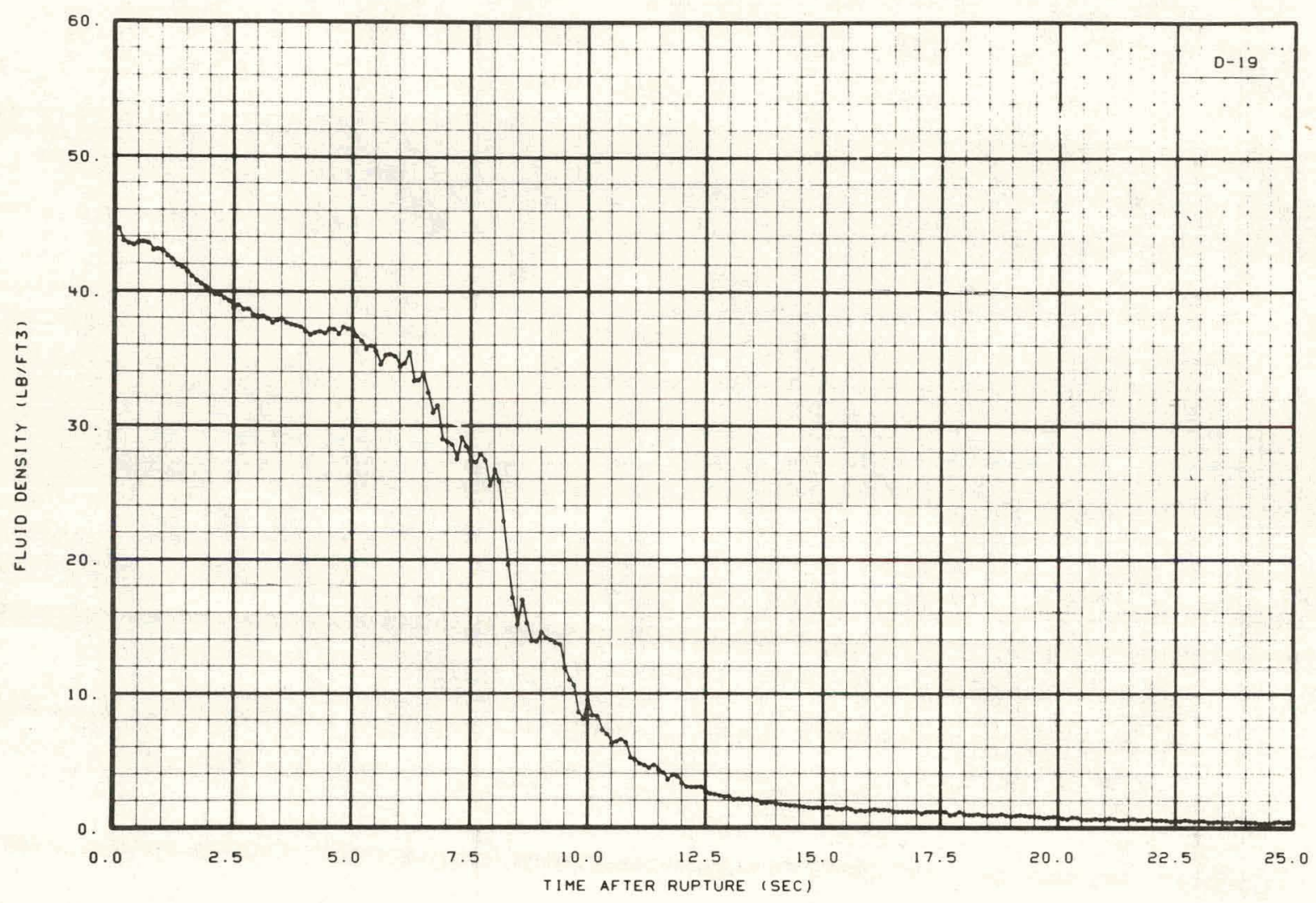

Fig. A-25 Pump outlet fluid density -- Test 1010.

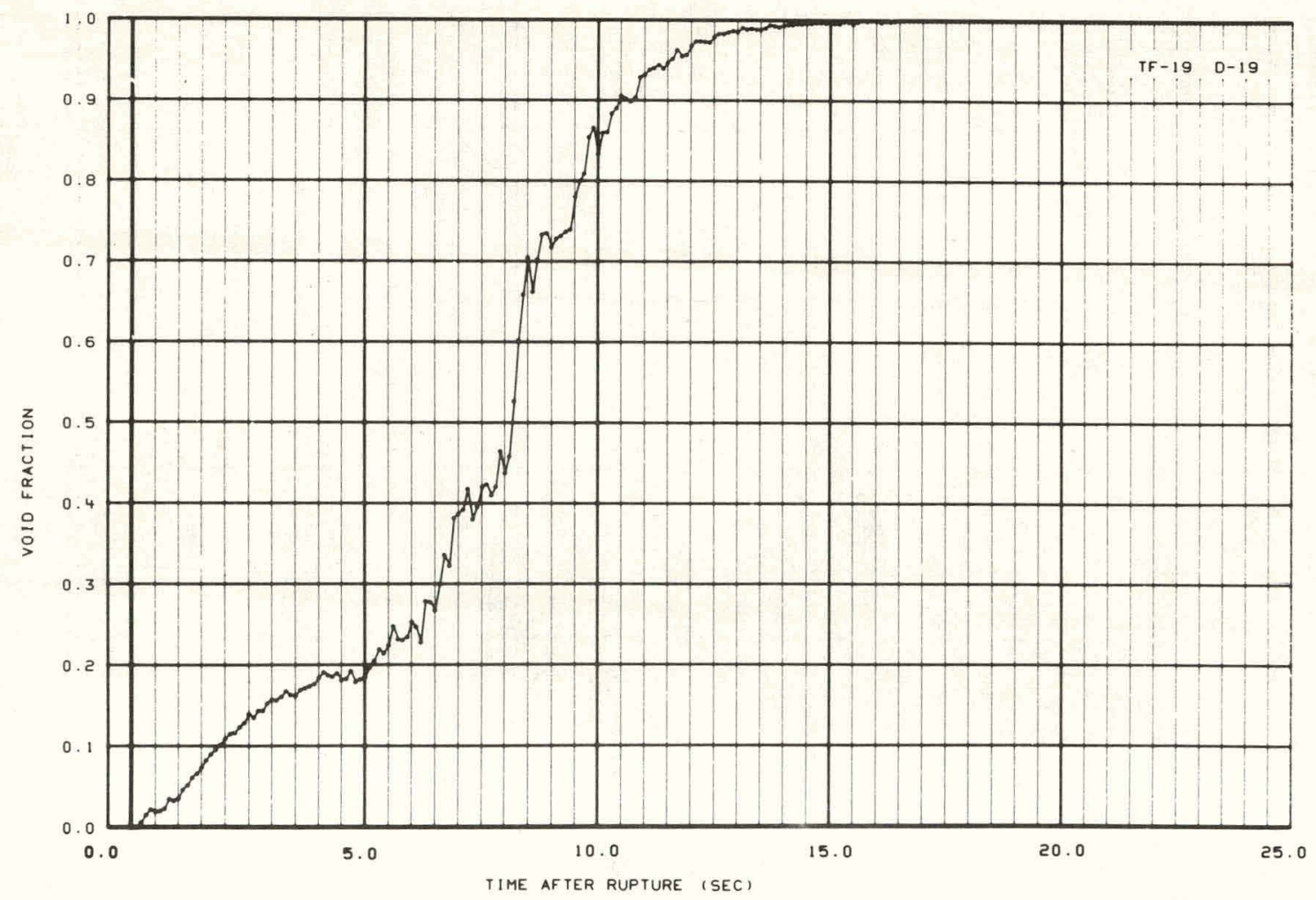

Fig. A-26 Pump outlet void fraction -- Test 1010. 


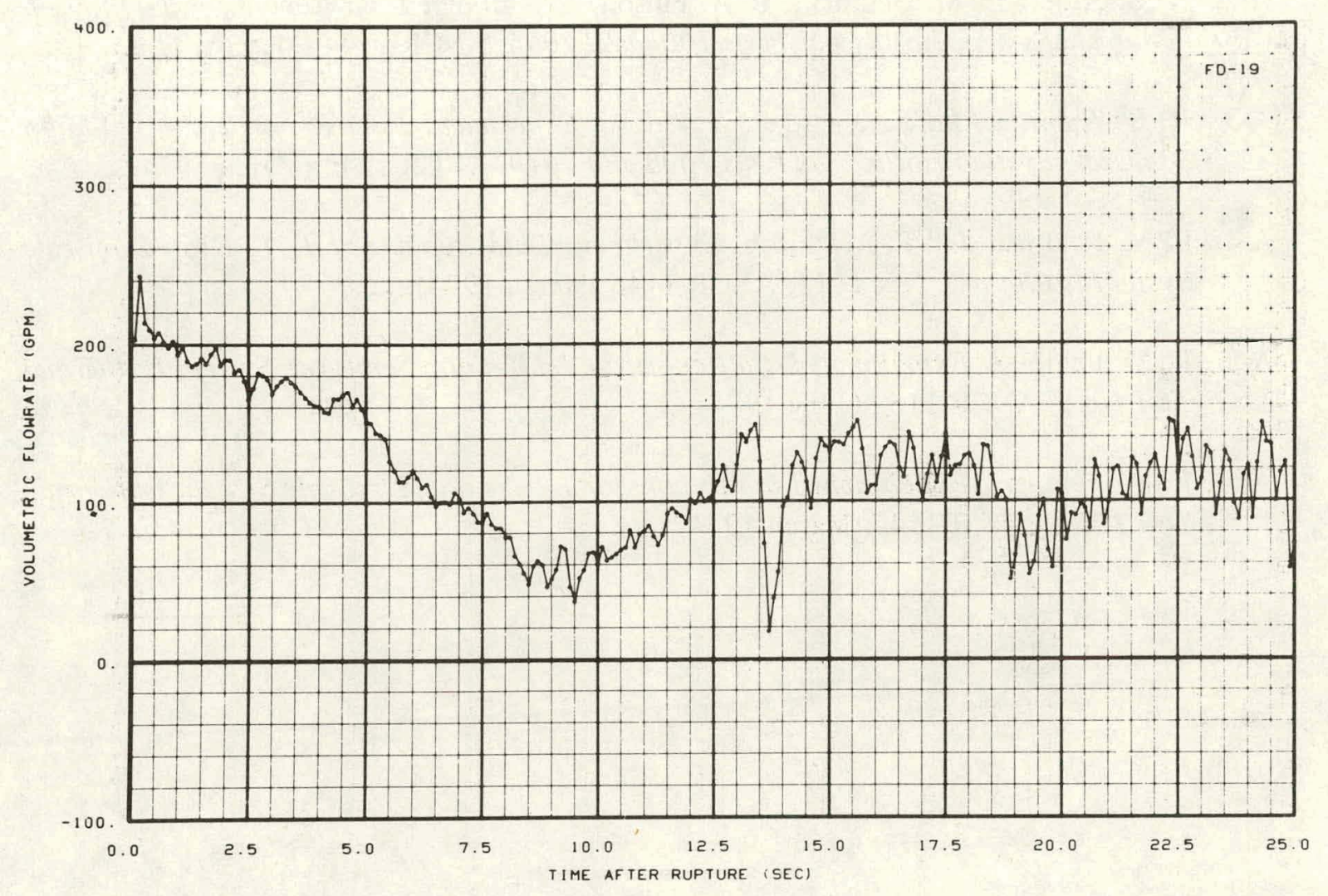

Fig. A-27 Pump outlet volumetric flow rate -- Test 1010. 


\section{REFERENCES}

A-1. R. S. Alder, E. M. Feldman, D. J. Olson, P. A. Pinson, Experiment Data Report for 1-1/2-Loop Semiscale System Isothermal Tests 1001, 1002, and 1003, ANCR-1148 (May 1974).

A-2. R. S. Alder, E. M. Feldman, P. A. Pinson, Experiment Data Report for 1-1/2-Loop Semiscale System Isothermal Tests 1004 and 1006, ANCR-1144 (March 1974).

A-3. R. S. Alder, E. M. Feldman, P. A. Pinson, Experiment Data Report for 1-1/2-Loop Semiscale System Isothermal Tests 1008 and 1010, ANCR-1145 (March 1974).

A-4. E. M. Feldman and P. A. Pinson, Experiment Data Report for 1-1/2-Loop Semiscale System Isothermal Test 1011, ANCR-1146 (March 1974).

A-5. E. M. Feldman, Experiment Data Report for 1-1/2-Loop Semiscale System Isothermal Test 1009, ANCR-1152 (June 1974).

A-6. E. M. Feldman, Experiment Data Report for 1-1/2-Loop Semiscale System Isothermal Test 1014, ANCR-1147 (March 1974). 


\section{APPENDIX B}

THE EFFECTS OF FLOW REGINE ON MEASURED DENSITY AND THE METHOD

OF OBTAINING AN EFFECTIVE DENSITY AT THE PUMP INLET 


\section{THIS PAGE WAS INTENTIONALLY LEFT BLANK}




\title{
APPENDIX B
}

\section{THE EFFECTS OF FLOW REGIME ON MEASURED DENSITY AND THE METHOD}

\author{
OF OBTAINING AN EFFECTIVE DENSITY AT THE PUMP INLET
}

\begin{abstract}
An evaluation of pump performance under two-phase fluid conditions is highly dependent on knowledge of the two-phase density entering the pump. A discussion of the limitations of the single-beam gamma attenuation method of obtaining the density when the flow is other than homogeneous and of the technique used to obtain an effective density on the basis of an assumed flow regime are presented in this appendix.
\end{abstract}

\section{FLOW REGIME AND MEASURED DENSITY}

The gamma attenuation method of using a single vertically oriented beam for measuring the density of a flowing fluid in a circular conduit inherently includes the assumption of a homogeneous flow regime. The measurement is made across a small cross section and the results are interpreted assuming the density is uniform across the total cross section of the pipe. If the flow regime is other than homogeneous, such as annular or stratified, then the effect of the curvature of the pipe must be considered. If, for example, the gamma attenuation technique with the assumption of homogeneous flow is used in a situation in which pure annular flow is present, the relationship between the void fraction obtained from the measurement and the actual void fraction can be determined in the following manner.

The following geometrical representation of pure annular flow within a circular pipe shown in Figures B-1 and B-2 is considered.

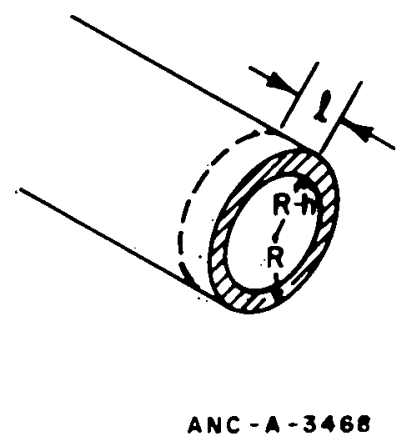

Fig. B-1 Representalion of annular flow in pipe.

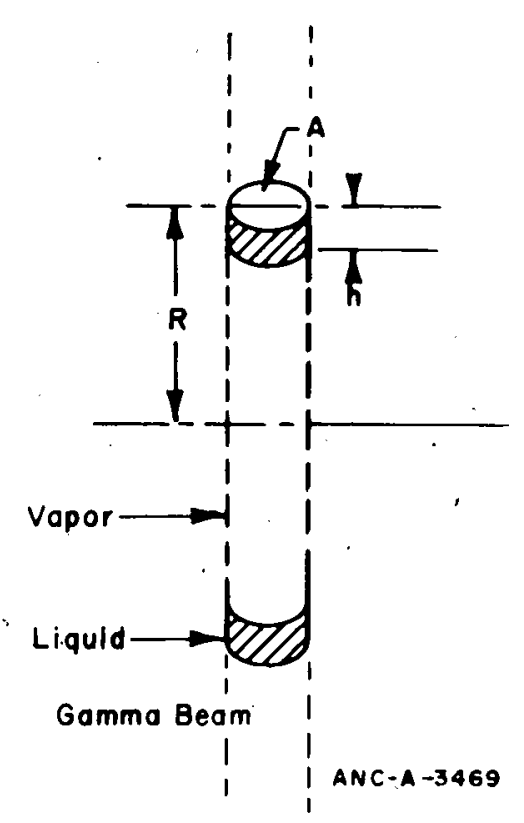

-Fig. B-2 Path traversed by gamma beam through pipe containing annular flow. 
Where
A $=$ area of beam cross section $\left(\mathrm{ft}^{2}\right)$
$\mathrm{R} \quad=\quad$ pipe radius $(\mathrm{ft})$
$\mathrm{h}=$ annular film thickness (ft).

On the basis of the measured density, $\rho_{\mathrm{m}}$, the following expression is obtained:

$$
\rho_{m} V=\rho_{g} V_{g}+\rho_{\ell} V_{\ell}
$$

where from Figure B-2

$$
\begin{array}{ll}
\mathrm{V} & =\text { total volume }\left(\mathrm{ft}^{3}\right)=2 \mathrm{RA} \\
\mathrm{V}_{\mathrm{g}}= & \text { volume of vapor phase }\left(\mathrm{ft}^{3}\right)=2 \mathrm{~A}(\mathrm{R}-\mathrm{h}) \\
\mathrm{V}_{\ell}= & \text { volume of liquid phase }\left(\mathrm{ft}^{3}\right)=2 \mathrm{Ah} \\
\rho_{\mathrm{g}}= & \text { density of vapor phase }\left(\mathrm{lb} / \mathrm{ft}^{3}\right) \\
\rho_{\ell}= & \text { densily of liquid phasc }\left(\mathrm{lb} / \mathrm{ft}^{3}\right) .
\end{array}
$$

Substitution of Equations (B-2), (B-3), and (B-4) into Equation (B-1) results in:

$$
\rho_{\mathrm{m}}=\rho_{\mathrm{g}}(1-\mathrm{h} / \mathrm{R})+\rho_{\ell}[1-(1-\mathrm{h} / \mathrm{R})] \text {. }
$$

The relationship between void fraction and density is defined as:

$$
\rho=\rho_{g} \alpha+\rho_{\ell}(1-\alpha)
$$

Therefore,

$$
\alpha=\alpha_{\mathrm{m}}=(1-h / R)
$$

where $a_{m}$ is defined as the void fraction obtained from the measurement of average density, assuming homogeneous flow.

To determine the expression for the actual density, $\rho_{\mathrm{a}}$, Equation (B-1) is rewritten as

$$
\rho_{\mathrm{a}} \mathrm{V}=\rho_{\mathrm{g}} \mathrm{V}_{\mathrm{g}}+\rho_{\ell} \mathrm{V}_{\ell}
$$


However, from Figure B-1, consideration of a finite circular element of length, $\ell$, results in:

$$
\begin{aligned}
& V=\pi R^{2} \ell \\
& V_{g}=\pi(R-h)^{2} \ell \\
& V_{\ell} \quad \pi R^{2} \ell-\pi(R-h)^{2} \ell .
\end{aligned}
$$

Substitution of Equations (B-9), (B-10), and (B-11) into-Equation (B-8) results in:

$$
\rho_{a}=\rho_{g}(1-h / R)^{2}+\rho_{\ell}\left[1-(1-h / R)^{2}\right] .
$$

Therefore,

$$
\alpha=\alpha_{a}=(1-h / R)^{2}
$$

Or,

$$
\alpha_{\mathrm{a}}=\left(\alpha_{\mathrm{m}}\right)^{2}
$$

From.Equation (B-5),

$$
\alpha_{m}=\frac{\rho_{m}-\rho_{\ell}}{\rho_{g}-\rho_{\ell}} \text {. }
$$

Substitution of Equation (B-15) into Equation (B-12) results in a final expression relating the measured and actual densities in a pure annular flow regime:

$$
\rho_{\mathrm{a}}=\rho_{\mathrm{g}}\left(\frac{\rho_{\mathrm{m}}-\rho_{\ell}}{\rho_{\mathrm{g}}-\rho_{\ell}}\right)^{2}+\rho_{\ell}\left[1-\left(\frac{\rho_{\mathrm{m}}-\rho_{\ell}}{\rho_{\mathrm{g}}-\rho_{\ell}}\right)^{2}\right] .
$$

The graphical interpretation of the analysis presented for the case of pure annular flow and of the results of a.similar analysis for the case of pure stratified flow are given in Figures B-3 and B-4, respectively, for 900 psia. 


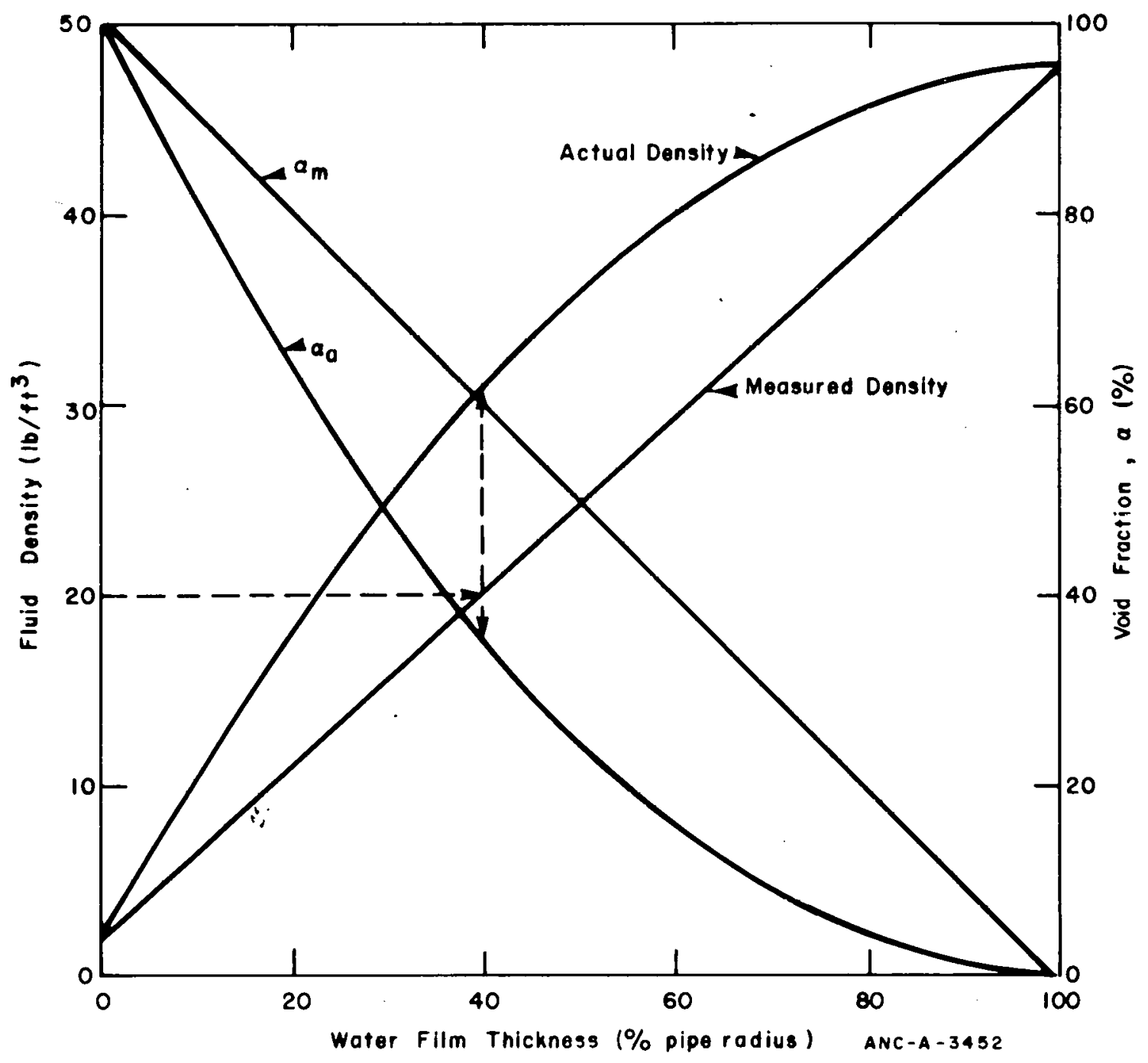

Fig. B-3 Fluid density and void fraction versus film thickness for pure annular flow.

In summary, with the flow regime specified as annular or stratified, the procedure required to correct the density and obtain an actual local average density and void fraction is straightforward using Figures B-3 and B-4. For example, if the measured density is 20 $\mathrm{lb} / \mathrm{ft}^{3}$ and annular flow is indicated, the actual local average density is $30.7 \mathrm{lb} / \mathrm{ft}^{3}$ and the void fraction is $35.5 \%$, from Figure B-3.

\section{EFFECTIVE DENSITY}

Use of an effective density concept is considered required to properly correlate two-phase pump performance data when separated flow regimes exist. The objective is to define a density representative of the physical situation that would occur if the volumetric fluxes of the individual phases are completely mixed as they reach the pump impeller. The 


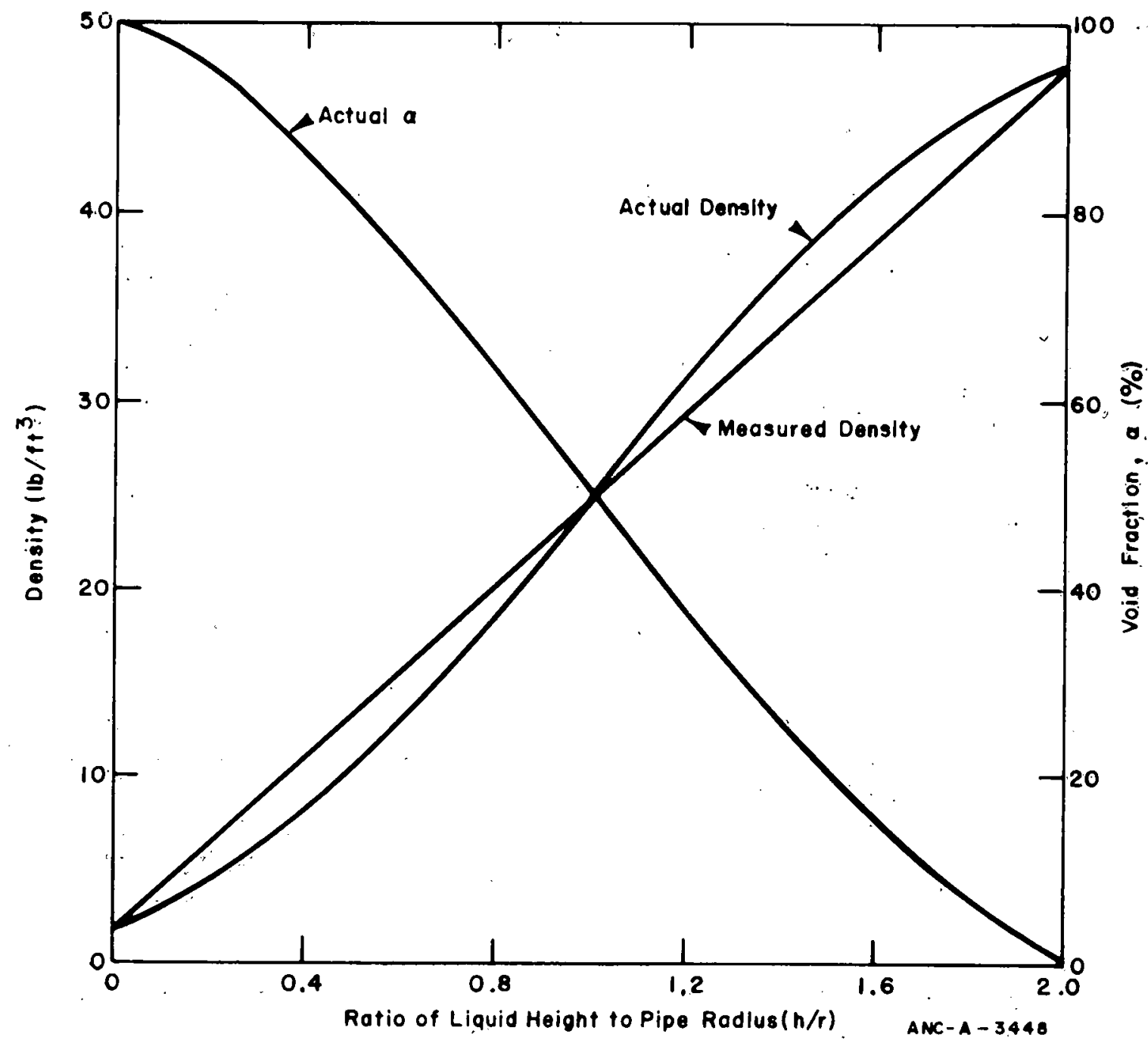

Fig. B-4. Fluid density and void fraction versus liquid height for pure stratified flow.

effective density used in the semiscale pump analysis related the saturated phase densities, the upsteam void fraction (calculated on the basis of an assumed flow regime as described), and the relative velocity (slip ratio) between the phases. The effective density was obtained in the following manner.

The total mass flow rate, $\dot{\mathrm{m}}_{\mathrm{T}}$, is known and is equal to the sum of the individual mass flow rates of the vapor; $\dot{\mathrm{m}}_{\mathrm{g}}$, and liquid, $\dot{\mathrm{m}}_{\ell}$ :

Since

$$
\dot{\mathrm{m}}_{\mathrm{T}}=\dot{\mathrm{m}}_{\mathrm{g}}+\dot{\mathrm{m}}_{\ell} \text {. }
$$

$$
\dot{\mathrm{m}} .=\rho \cdot \mathrm{A} \mathrm{v}
$$

then

$$
\dot{i i}_{\mathrm{T}}=\rho_{g} \mathrm{~A}_{\mathrm{g}} \mathrm{v}_{\mathrm{g}}+\rho_{\ell} \mathrm{A}_{\ell} \mathrm{v}_{\ell}
$$


where

$$
\begin{aligned}
& \rho \quad=\text { density }\left(\mathrm{lb} / \mathrm{ft}^{3}\right) \\
& \mathrm{A} \quad=\quad \text { cross-sectional area }\left(\mathrm{ft}^{2}\right) \\
& \mathrm{v}=\text { velocity }(\mathrm{ft} / \mathrm{sec}) \\
& \mathrm{g} \quad=\quad \text { subscript, vapor phase } \\
& \ell \quad=\quad \text { subscript, liquid phase. }
\end{aligned}
$$

Multiplying and dividing the right-hand side of Equation (B-17) by the total pipe cross-sectional area, $\mathrm{A}_{\mathrm{T}}$, results in:

$$
\dot{\mathrm{m}}_{\mathrm{T}}=\mathrm{A}_{\mathrm{T}} \frac{\rho_{\mathrm{g}} \mathrm{v}_{\mathrm{g}} \mathrm{A}_{\mathrm{g}}}{\mathrm{A}_{\mathrm{T}}}+\frac{\rho_{\ell} \mathrm{v}_{\ell} \mathrm{A}_{\ell}}{\mathrm{A}_{\mathrm{T}}} .
$$

Since

$$
\begin{aligned}
& \alpha=\mathrm{A}_{\mathrm{g}} / \mathrm{A}_{\mathrm{T}} \text { (upstream local average void fraction), } \\
& \dot{\mathrm{m}}_{\mathrm{T}}=\mathrm{A}_{\mathrm{T}}\left[\rho_{\mathrm{g}} \alpha \mathrm{v}_{\mathrm{g}}+\rho_{\ell}(1-\alpha) \mathrm{v}_{\ell}\right]
\end{aligned}
$$

If the pump responds to an effective density then the total volumetric flow rate can be expressed as:

$$
Q_{T}=\frac{\dot{\mathrm{m}}_{\mathrm{T}}}{\rho_{\text {eff }}}=\frac{\mathrm{A}_{\mathrm{T}}}{\rho_{\text {eff }}}\left[\rho_{\mathrm{g}} \alpha \mathrm{v}_{\mathrm{g}}+\rho_{\ell}(1-\alpha) \mathrm{v}_{\ell}\right]
$$

The total volumetric flow rate into the pump can also be expressed as the sum of the volumetric flow rates of the phases:

$$
\begin{aligned}
\mathrm{Q}_{\mathrm{T}}{ }^{\prime} & =\mathrm{Q}_{\mathrm{g}}+\mathrm{Q}_{\ell} \\
& =\mathrm{A}_{\mathrm{g}} \mathrm{v}_{\mathrm{g}}+\mathrm{A}_{\ell} \mathrm{v}_{\ell} \\
& =\mathrm{A}_{\mathrm{T}}\left(\frac{\mathrm{A}_{\mathrm{g}}}{\mathrm{A}} \mathrm{v}_{\mathrm{g}}+\frac{\mathrm{A}_{\ell}}{\mathrm{A}_{\mathrm{T}}} \mathrm{v}_{\ell}\right) \\
& =\mathrm{A}_{\mathrm{T}}\left[\alpha \mathrm{v}_{\mathrm{g}}+(1-\alpha) \mathrm{v}_{\ell}\right] .
\end{aligned}
$$


Equating Equations (B-19) and (B-20) results in:

$$
\rho_{\text {eff }}=\frac{\rho_{g} v_{g} \alpha+\rho_{\ell} v_{\ell}(1-\alpha)}{v_{g} \alpha+v_{\ell}(1-\alpha)} .
$$

Multiplying and dividing Equation $(\mathrm{B}-21)$ by $\mathrm{v}_{\ell}$ produces

$$
\rho_{\text {eff }}=\frac{\rho_{g}\left(v_{g} / v_{\ell}\right) \alpha+\rho_{\ell}(1-\alpha)}{\left(v_{g} / v_{\ell}\right) \alpha+(1-\alpha)} .
$$

Finally

$$
\rho_{\text {eff }}=\frac{\rho_{g} s \alpha+\rho_{\ell}(1-\alpha)}{s \alpha+(1-\alpha)}
$$

where the definition of slip ratio, $S=v_{g} / v_{\ell}$, has been employed.

A flow regime map presented by Govier and Omer ${ }^{[B-1]}$ was utilized to obtain an approximate value for the slip ratio for use in Equation (B-23). The flow regime map is plotted as holdup, or slip ratio, as a function of the superficial velocities of the vapor, ${ }^{\mathrm{V}_{\mathrm{S}}} \mathrm{g}_{\mathrm{g}}$, and liquid, ${ }^{\mathrm{S}_{\ell}}{ }_{\ell}$, phases. The superficial velocities are defined as

$$
\begin{aligned}
& v_{S_{\ell}}=\frac{\dot{\mathrm{m}}_{\mathrm{T}}(1-\mathrm{x})}{\rho_{\ell} \mathrm{A}_{\mathrm{T}}} \text { (1iquid phase) } \\
& \mathrm{v}_{\mathrm{S}}=\frac{\dot{\mathrm{m}}_{\mathrm{T}} \mathrm{x}}{\rho_{\mathrm{g}} \mathrm{A}_{\mathrm{T}}} \text { (vapor phase) }
\end{aligned}
$$

where the local average quality, $\mathrm{x}$, for a separated flow condition is defined as

$$
x=\left[1+\frac{1}{S} \underset{\rho_{g}}{\left(\frac{\ell}{\rho}\right)} \quad\left(\frac{1-\alpha}{\alpha}\right)\right]^{-1}
$$

The following procedure was used to obtain the slip ratio:

(1) A flow regime is assumed

(2) A local average void fraction $(\alpha)$ corresponding to the assumed flow regime is determined 
(3) A slip ratio is assumed

(4) Equation (B-26) is solved for $\mathrm{x}$

(5) Equations (B-24) and (B-25) are solved for ${ }^{v_{S}} S_{\ell}$ and ${ }^{v} S_{g}$

(6). $S$ is obtained from a flow regime map

(7) Iterations are conducted until a compatible combination of $\mathrm{v}_{\mathrm{S}_{\mathrm{g}}}$, ${ }^{\mathrm{S}_{\ell}}$, and $\mathrm{S}$ are
obtained.

With the slip ratio obtained, Equation (B-23) is solved for $\rho_{\text {eff }}$ and that value is used in the calculation of pump head and volumetric flow rate. Table B-I presents a listing of the data presented in Section III-2 of this report with the probable flow regime as determined by the method described. 
$\underline{\text { TABLE B-I }}$

FLOW REGIME TYPE FROM EFFECTIVE DENSITY METHOD

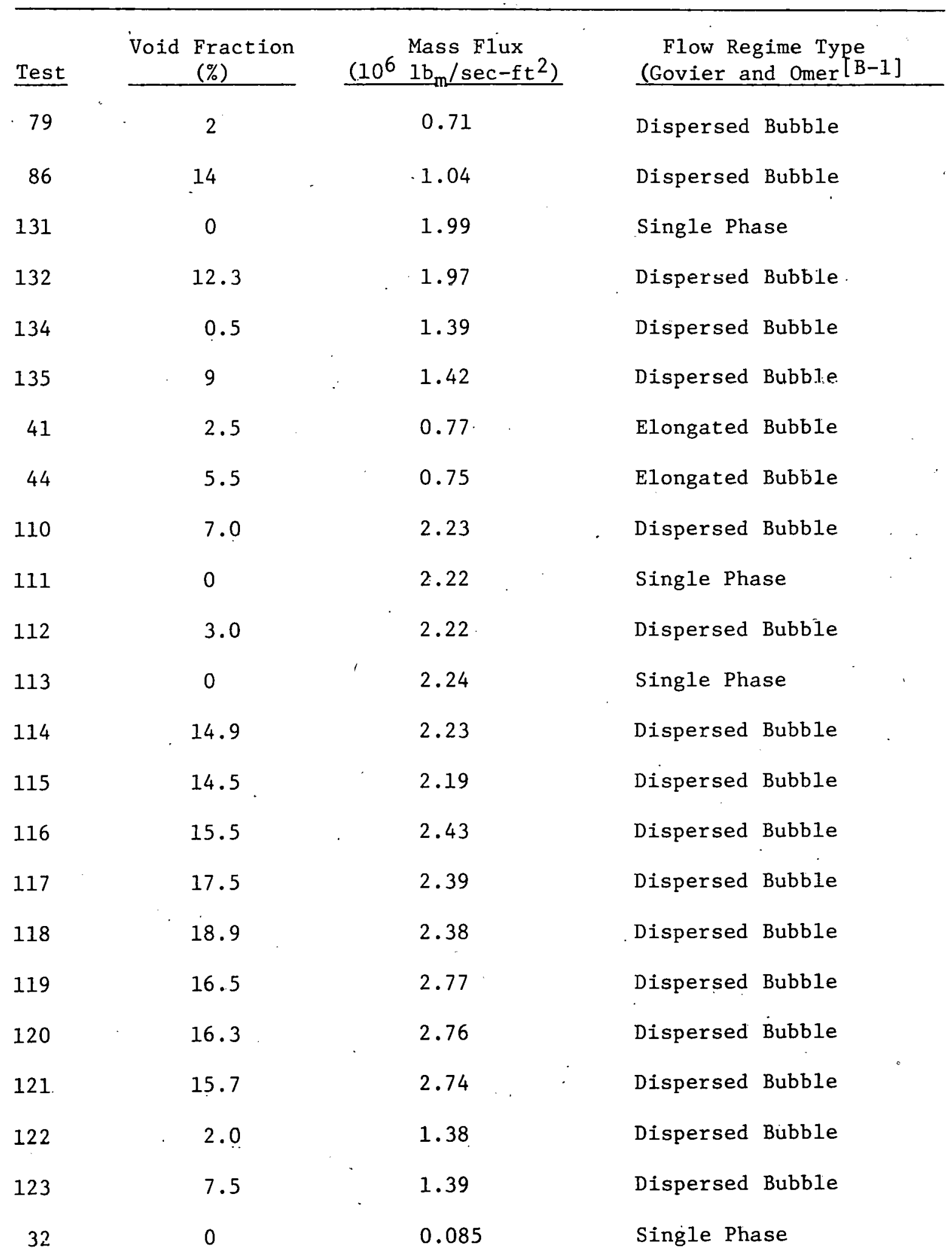


TABLE B-I (cont'd)

\begin{tabular}{|c|c|c|c|c|c|}
\hline Test & Void & $\begin{array}{l}\text { Fraction } \\
(\%) \\
\end{array}$ & $\left(10^{6}\right.$ & $\begin{array}{l}\text { Mass Flux } \\
\left.1 b_{m} / \sec -f t^{2}\right)\end{array}$ & $\begin{array}{l}\text { Flow Regime Type } \\
\text { (Govier and Omer [B-1] }\end{array}$ \\
\hline 80 & & 0 & & 0.67 & Single Phase \\
\hline 84 & & 0 & & 0.36 & Single Phase \\
\hline 137 & & 0 & & $0.92^{\circ}$ & Single Phase \\
\hline 140 & & 8.0 & & 0.11 & Elongatod Buhhip \\
\hline 141 & & 11.0 & & 0.92 & Elongated Bubble \\
\hline 45 & & 10.0 & & 0.77 & Elongated Bubble \\
\hline 125 & & 0 & & 1.33 & Single Phase \\
\hline 126 & & 8.0 & & 1.31 & Dispersed Bubble \\
\hline 127 & & 10.0 & & 1.30 & Dispersed Bubble \\
\hline 128 & & 0 & & 0.87 & Single Phase \\
\hline 129 & & 12.0 & & 0.87 & Elongated Bubble \\
\hline 37 & & 0 & & 0.14 & Single Phase \\
\hline 38 & & 0 & & 0.14 & Single Phase \\
\hline 39 & & 0 & & 0.14 & Single Phase \\
\hline 66 & & 0.5 & & 0.65 & Dispersed Bubble \\
\hline 7 & & 30.0 & & 2.58 & Dispersed Bubble \\
\hline 78 & & 20.0 & & 0.58 & Elongated Bubble \\
\hline 87 & & 34.0 & & 0.96 & Slug \\
\hline 88 & & 23.0 & & 1.09 & Slug \\
\hline 89 & & 23.0 & & 1.23 & Elongated Bubble \\
\hline 133 & & 21.0 & & 1.92 & Dispersed Bubble \\
\hline 136 & & $20.0^{-}$ & & 1.40 & Elongated Bubble \\
\hline 16 & & 25.4 & & 2.07 & Dispersed Bubble \\
\hline 96 & & 33.0 & & 1.31 & slug \\
\hline
\end{tabular}


TABLE B-I (cont'd)

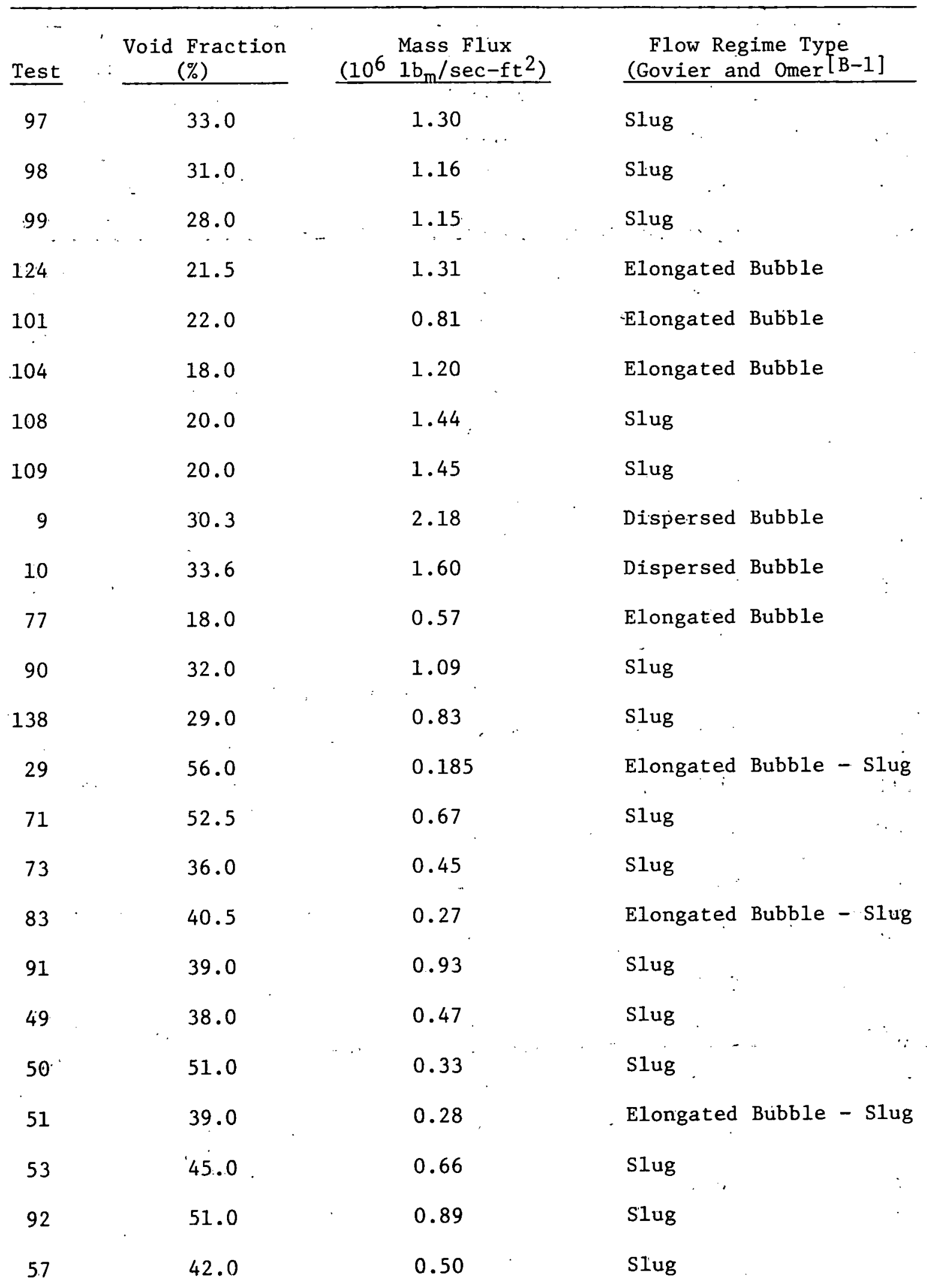


TABLE B-I (cont'd)

\begin{tabular}{|c|c|c|c|}
\hline Test & $\begin{array}{l}\text { Void Fraction } \\
(\%) \\
\end{array}$ & $\begin{array}{ll} & \text { Mass Flux } \\
\left(10^{6} \quad 1 b_{\mathrm{m}} / \mathrm{sec}-\mathrm{ft}^{2}\right) \\
\end{array}$ & $\begin{array}{l}\text { Flow Regime Type } \\
\text { (Govier and Omer }[B-1]\end{array}$ \\
\hline 58 & 49.0 & 0.43 & Slug \\
\hline 61 . & 48.0 & 0.28 & Elongated Bubble - Slug \\
\hline $10 \dot{6}$ & 36.0 & 0.98 & Slug \\
\hline 107 & 36.5 & 0.98 & Slug \\
\hline 30 & 54.5 & 0.18 & Elongated Bubble - Slug \\
\hline 31 & 53.0 & 0.15 & Elongated Bubble \\
\hline 69 & 43.0 & 0.78 & Slug \\
\hline 72 & 37.5 & 0.53 & Slug \\
\hline 82 & 56.0 & 0.26 & Slug \\
\hline 35 & 57.0 & 0.17 & Elongated Bubble - Slug \\
\hline 48 & 48.5 & 0.38 & Slug \\
\hline 52 & 61.0 & 0.145 & Slug \\
\hline 60 & 53.0 & 0.38 & slug \\
\hline 192 & 91.0 & 0.11 & Wavy - Annular Mist \\
\hline 194 & 83.0 & 0.087 & Wavy \\
\hline 195 & 88.0 & 0.072 & Wavy \\
\hline 198 & 90.0 & 0.091 & Wavy \\
\hline 182 & 84.0 & 0.15 & Wavy - Annular Mist \\
\hline 184 & 89.0 & 0.09 & Wavy - Annular Mist \\
\hline 185 & 85.0 & 0.082 & Wavy \\
\hline 189 & 91.0 & 0.062 & Wavy - Annular Mist \\
\hline 62 & 66.5 & 0.17 & Slug \\
\hline 170 & 80.0 & 0.14 & Wavy - slug \\
\hline 172 & 78.0 & 0.13 & Wavy \\
\hline
\end{tabular}


TABLE B-I (cont'd)

\begin{tabular}{|c|c|c|c|c|}
\hline$\underline{\text { Test }}$ & $\begin{array}{l}\text { Void Fraction } \\
(\%) \\
\end{array}$ & $\left(10^{6}\right.$ & $\begin{array}{l}\text { Mass Flux } \\
\left.1 b_{m} / \sec ^{2} t^{2}\right)\end{array}$ & $\begin{array}{l}\text { Flow Regime Type } \\
\text { (Govier and Omer [B-1] }\end{array}$ \\
\hline 173 & 82.0 & & 0.10 & Wavy \\
\hline 174 & 80.5 & & 0.11 & Wavy \\
\hline 176 & 79.0 & & 0.11 & Wavy \\
\hline $17: 8$ & 81.5 & & 0.0 .58 & Wavy \\
\hline 191 & 91.5 & & 0.11 & Wavy - Annular Mist \\
\hline $19.3^{\circ}$ & 88.0 & 1 & 0.095 & Wavy \\
\hline 197 & 88.5 & & 0.081 & Wavy \\
\hline 186 & .86 .0 & & 0.085 & Wavy \\
\hline 188 & 93.5 & & 0.063 & Wavy - Annular Mist \\
\hline 190 & 91.5 & & 0.042 & Wavy \\
\hline 17.1 & 76.0 & & 0.12 & Wavy - Slug \\
\hline 17.9 & 85.0 & & 0.06 & Wavy \\
\hline 180 & 72.5 & & 0.054 & Wavy \\
\hline 181 & 77.0 & & 0.035 & Wavy \\
\hline
\end{tabular}




\section{REFERENCE}

1. G. W. Govier and K. Aziz, The Flow of Complex Mixtures in Pipes, Van Nostrand Reinhold Company (1972).

D 
APPENDIX C ANALYSIS OF SEMISCALE TRANSIENT DATA

CONSIDERING THE EFFECTS OF IMPELLER FLUID INERTIA 


\section{THIS PAGE \\ WAS INTENTIONALLY \\ LEFT BLANK}




\section{APPENDIX C \\ ANALYSIS OF SEMISCALE TRANSIENT DATA CONSIDERING THE EFFECTS OF IMPELLER FLUID INERTIA}

During a simulated LOCA, transient fluid accelerations in the Mod-1 pump and the pump inlet and discharge lines may influence the transient head performance of the pump. The following analysis was performed to determine the significance of the transient acceleration relative to the steady state pump performance.

The following simplified geometrical representation of a centrifugal pump impeller is considered.

where

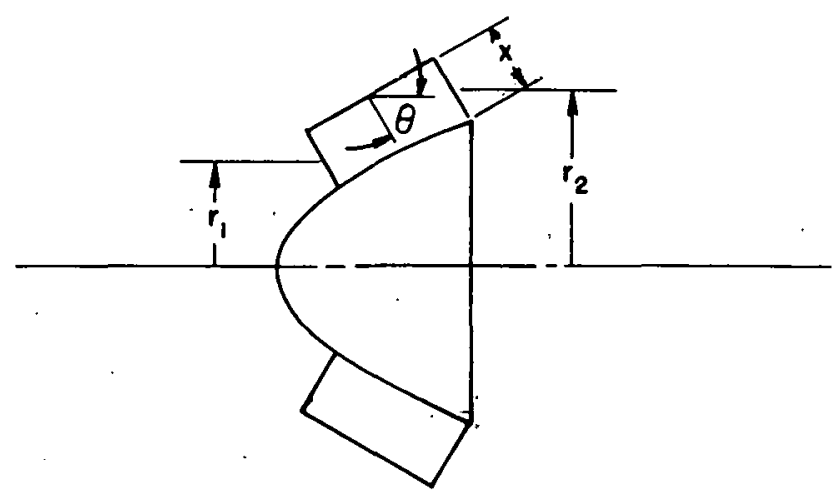

$$
\begin{aligned}
& \mathrm{r}_{1}=\text { impeller blade inlet diameter }(\mathrm{ft}) \\
& \mathrm{r}_{2}=\text { impeller blade discharge diameter }(\mathrm{ft}) \\
& \mathrm{x}=\text { impeller blade height }(\mathrm{ft}) \\
& \Theta \quad=\quad \text { impeller shroud angle (degrees). }
\end{aligned}
$$

The total surface transient force acting on the impeller can be expressed in integral form as

$$
F_{\text {trans }}=\frac{\partial}{\partial t} \int \frac{1}{g_{c}} v \rho d V
$$

where

$$
\begin{array}{ll}
\mathrm{g}_{\mathrm{C}} & =\text { gravitational acceleration }\left(\mathrm{ft} / \mathrm{sec}^{2}\right) \\
v & =\text { velocity }(\mathrm{ft} / \mathrm{sec}) \\
\rho & =\text { density }\left(\mathrm{lb} / \mathrm{ft}^{3}\right)
\end{array}
$$




$$
\mathrm{V}=\text { volume }\left(\mathrm{ft}^{3}\right)
$$

Specifically, the transient differential pressure across the pump, $\Delta \mathrm{P}_{\text {trans }}$ or $\mathrm{F}_{\text {trans }} /$ Area, is the desired quantity. From geometrical considerations,

$$
\begin{aligned}
& A=2 \pi r \times \text { (area normal to flow) } \\
& \mathrm{dV}=2 \pi \mathrm{r} \times \mathrm{dr} / \cos \Theta \text { (elemental volume) }
\end{aligned}
$$

Therefore, Equation (C-1) becomes

$$
F_{\text {trans }}=\frac{\partial}{\partial t} \int \frac{1}{g_{c}} \vee \rho 2 \pi r x d r / \cos \theta
$$

or

$$
\Delta \mathrm{P}_{\text {trans }}=\frac{\mathrm{F}_{\text {trans }}}{2 \pi \mathrm{r} x}=\frac{\partial}{\partial t} \int \frac{1}{\mathrm{~g}_{c}} \nu \rho \mathrm{dr} / \cos \theta .
$$

From consideration of the impeller vector diagram (without consideration for slip),

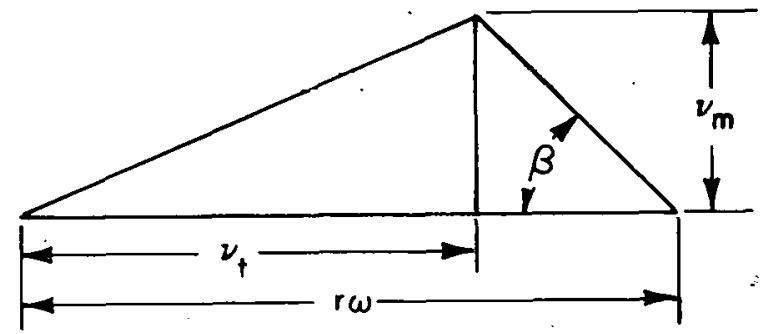

$$
\nu=\nu_{t}=r \omega-\nu_{m} \cdot \cot \beta .
$$

With the assumption of incompressible fluid and parallel impeller shrouds, from the continuity law:

$$
A_{1} v_{m_{1}}=A_{2} v_{m_{2}} \text { or } \quad r_{1} \nu_{m_{1}}=r_{2} \nu_{m_{2}}
$$

for any $r$ :

$$
v_{\mathrm{m}}=\frac{\mathrm{r}_{1} \nu_{\mathrm{m}_{1}}}{\mathrm{r}}
$$

but

$$
\nu_{\mathrm{m}_{1}}=\mathrm{Q} / \mathrm{A} \cdot
$$


Therefore,

$$
\nu_{m}=\frac{r_{1} Q}{r \cdot A_{1}}
$$

Substitution of Equations (C-3) and (C-4) into Equation (C-2) results in

$$
\Delta \mathrm{P}_{\text {trans }}=\frac{\partial}{\partial t} \int \frac{\rho}{g_{c}}\left(r \omega-\frac{r_{1} Q \cot B}{A_{1} r}\right) \frac{d r}{\cos \theta} .
$$

By assuming $\Theta$ and $\beta$ are constant and integrating between the limits of $r_{1}$ and $r_{2}$, Equation $(C-5)$ can be rewritten as

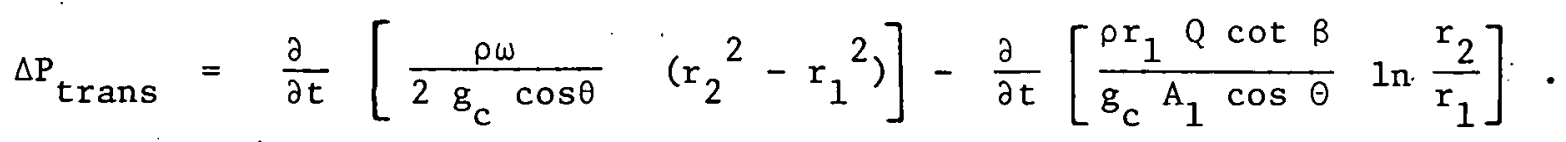

For a constant speed $(\omega)$ and varying flow rate (Q), Equation (C-6) becomes

$$
\Delta \mathrm{P}_{\text {trans }}=-\rho\left[\frac{\mathrm{r}_{1} \cot \beta}{\mathrm{g}_{\mathrm{c}} \mathrm{A}_{1} \cos \theta} \text { in } \frac{\mathrm{r}_{2}}{\mathrm{r}_{1}}\right] \frac{\mathrm{dQ}}{\mathrm{dt}} \text {. }
$$

Employing the relationship

$$
\Delta \mathrm{H}_{\text {trans }}=\frac{\Delta \mathrm{P}_{\text {trans }}}{\rho}
$$

results in :

$$
\Delta \mathrm{H}_{\text {trans }}=-\frac{\mathrm{r}_{1} \cot \beta}{\mathrm{g}_{\mathrm{c}} \mathrm{A}_{1} \cos \theta} \text { ln }\left(\frac{\mathrm{r}_{2}}{\mathrm{r}_{1}}\right) \frac{\mathrm{dQ}}{\mathrm{dt}}
$$

where

$$
\begin{array}{lll}
\beta & \equiv & \text { impeller blade angle (degrees) } \\
\omega & = & \text { impeller angular velocity (radians/sec) } \\
v_{\mathrm{m}} & = & \text { impeller median velocity }(\mathrm{ft} / \mathrm{sec}) \\
v_{\mathrm{t}} & = & \text { impeller tangential velocity }(\mathrm{ft} / \mathrm{sec}) \\
\mathrm{t} & = & \text { time }(\mathrm{sec}) \\
\mathrm{A} & = & \text { area normal to flow }\left(\mathrm{ft}^{2}\right) \\
\Delta \mathrm{H}_{\text {trans }} & =
\end{array}
$$




$$
\begin{array}{lll}
\Delta \mathrm{P}_{\text {trans }} & \left.=\text { inertia pressure (lb } / \mathrm{in} .^{2}\right) \\
\mathrm{Q} & =\text { flow rate }\left(\mathrm{ft}^{3} / \mathrm{sec}\right) .
\end{array}
$$

The inertia head due to fluid acceleration in the piping adjacent to the pump can be determined from the following expression:

$$
\Delta \mathrm{H}_{\text {trans }}=\frac{\mathrm{L}}{\mathrm{g}_{\mathrm{c}} \mathrm{A}} \quad \frac{\mathrm{dQ}}{\mathrm{dt}}
$$

Equations (C-7) and (C-8) are generally applicable to computations of fluid inertia terms for times after the pipe break occurs and subcooled decompression is complete. Table C-I presents the impeller and line geometry for the Mod-1 pump in the semiscale geometry, and the magnitude of the inertia head term for the impeller and lines. The effect of the impeller inertia head is less than that of the lines. Table C-II compares the impeller and

TABLE C-I

\begin{tabular}{|c|c|c|c|}
\hline \multirow[b]{2}{*}{ Parameter } & \multirow[b]{2}{*}{ Impeller } & \multicolumn{2}{|c|}{ Piping } \\
\hline & & Inlet & Discharge \\
\hline$r_{1}(f t)$ & 0.67 & & \\
\hline$r_{2}(f t)$ & 0.322 & & , \\
\hline$x_{1}(f t)$ & 0.058 & & \\
\hline$A_{1}\left(f t^{2}\right)$ & 0.061 & & \\
\hline B (degrees) & 25 & & \\
\hline$\theta$ (degrees) & 90 & & \\
\hline Maximum length ( $f t)$ & - & 9.75 & 10 \\
\hline ID (in.) & & 2.62 & 2.62 \\
\hline$A\left(f t^{2}\right)$ & & 0.0376 & 0.0376 \\
\hline Transient Inertia Head & & & \\
\hline$\Delta H_{\text {trans }}(f t)$ & $0.1207 \mathrm{dQ} / \mathrm{dt}$ & $8.1 \mathrm{dQ} / \mathrm{dt}$ & $8.3 \mathrm{dQ} / \mathrm{dt}$ \\
\hline
\end{tabular}

PUMP IMPELLER, INLET LINE, DISCHARGE LINE GEOMETRY, AND TRANSIENT INERTIA HEAD FOR THE MOD-1 SEMISCALE PUMP 


\section{TABLE C-II}

COMPARISON OF IMPELLER AND PUMP PIPING FLUID INERTIA HEADS IN SEMISCALE SYSTEM

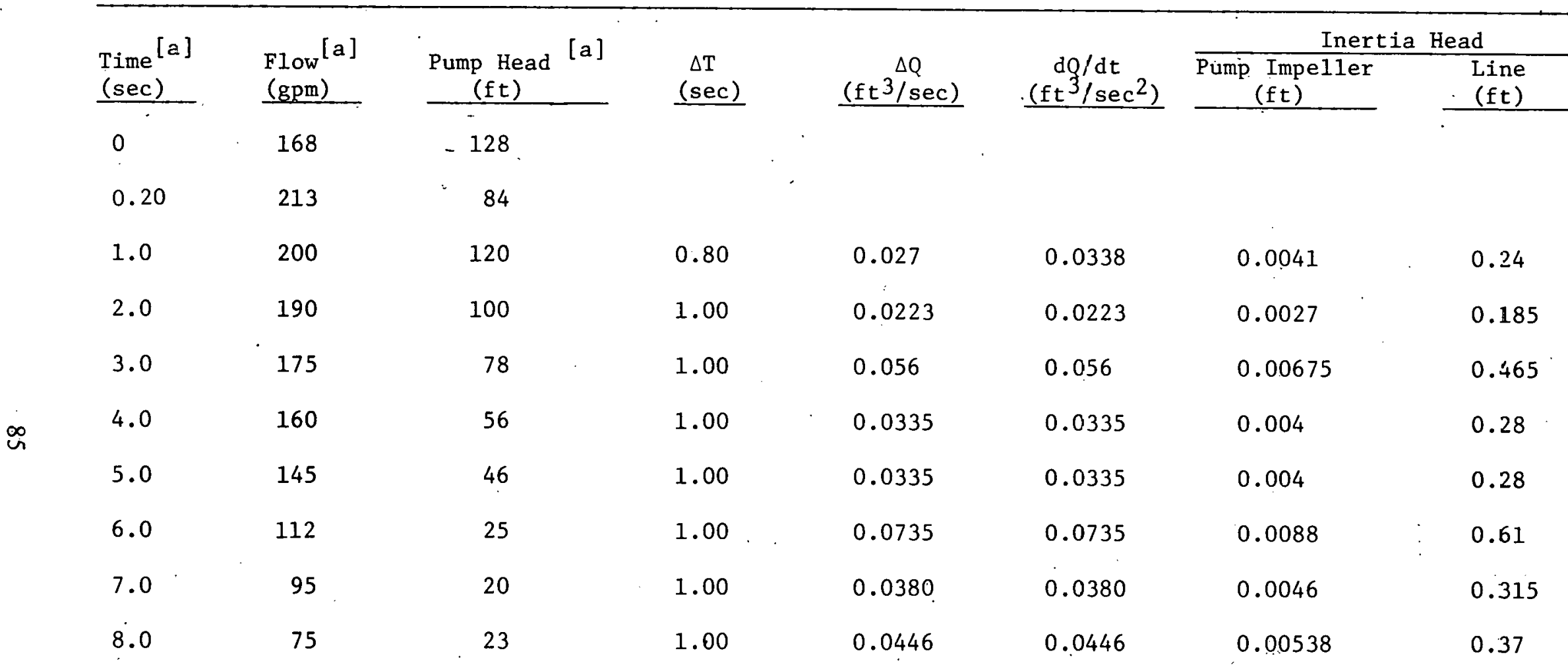

[a] For semiscale intact loop pump during isothermal blowdown transient Test 1010. 
piping fluid inertia heads for semiscale isothermal Test 1010. The results presented for this test are typical of transient results obtained from the isothermal test series. The results in Table C-II indicate that:

(1) The effect of fluid inertia in the impeller during a postulated LOCA, after the initial break, is very small and, therefore, can be neglected in an analysis without incurring significant error.

(2) The effect of fluid inertia in the pump inlet and discharge lines is also very small during a depressurization time of 15 to 20 seconds. 
3

I

1

,

1

- 